\title{
Article
}

\section{Contributions of Muscles and External Forces to Medial Knee Load Reduction Due to Osteoarthritis Braces}

Bandon, Scott CE, Brown, Marcus J, Clouthier, Allison L, Campbell, Aaron, Richards, James and Deluzio, Kevin J

Available at http://clok.uclan.ac.uk/28157/

Bandon, Scott CE, Brown, Marcus J, Clouthier, Allison L, Campbell, Aaron, Richards, James ORCID: 0000-0002-4004-3115 and Deluzio, Kevin J (2019)

Contributions of Muscles and External Forces to Medial Knee Load Reduction

Due to Osteoarthritis Braces. The Knee, 26 (3). pp. 564-577. ISSN 0968-0160

It is advisable to refer to the publisher's version if you intend to cite from the work. http://dx.doi.org/10.1016/j.knee.2019.04.006

For more information about UCLan's research in this area go to http://www.uclan.ac.uk/researchgroups/ and search for <name of research Group>.

For information about Research generally at UCLan please go to http://www.uclan.ac.uk/research/

All outputs in CLoK are protected by Intellectual Property Rights law, including Copyright law. Copyright, IPR and Moral Rights for the works on this site are retained by the individual authors and/or other copyright owners. Terms and conditions for use of this material are defined in the policies page. 


\section{CONTRibutions of Muscles AND EXTERnal ForCES to}

\section{MEDIAL KNEE LOAD REDUCTION DUE TO OSTEOARTHRITIS}

\section{BRACES}

Scott C.E. Brandon ${ }^{\mathrm{a}, \mathrm{b}, \mathrm{e} *}$, Marcus J. Brown ${ }^{\mathrm{a}, \mathrm{b}}$, Allison L. Clouthier ${ }^{\mathrm{a}, \mathrm{b}}$, Aaron Campbell ${ }^{\mathrm{b}, \mathrm{c}}$, Jim D. Richards $^{\mathrm{d}}$, and Kevin J. Deluzio ${ }^{\mathrm{a}, \mathrm{b}}$.

a Department of Mechanical and Materials Engineering, Queen's University, McLaughlin Hall, Kingston, Ontario, Canada. K7L 3N6

${ }^{\mathrm{b}}$ Human Mobility Research Centre, Kingston General Hospital, Angada 2, Kingston, Ontario, Canada. K7L 2V7

${ }^{c}$ Department of Surgery, Kingston General Hospital, Kingston, Ontario, Canada. K7L 2V7

d Allied Health Research Unit, University of Central Lancashire, Preston, UK, PR1 2HE

${ }^{\mathrm{e}}$ School of Engineering, University of Guelph, Guelph, Ontario, Canada, N1G 2W1

* corresponding author

scott.brandon@uoguelph.ca

$+15198244120 \times 52875$

An original article submitted to The Knee

Abstract: 243

Manuscript: 4361

Declarations of interest: none 


\section{Introduction}

Knee osteoarthritis is a disease characterized by progressive degradation of articular cartilage via mechanical forces [1]. In the United States alone, more than 9 million people suffer pain and reduced mobility as a result of knee osteoarthritis, with an economic burden exceeding $\$ 100$ billion [2]. Additionally, the number of patients who require treatment but are unsuitable for joint replacement surgery is projected to rise; bridging of this treatment gap is clinically important [3]. Osteoarthritis progression may be inhibited by reducing exposure to large compressive loads [4], particularly on the medial side where damage is most common [5]. Consequently, interventions such as knee braces have been developed to postpone disease progression by reducing medial knee loads.

Unfortunately, knee braces are not uniformly effective. In one study, $25 \%$ of participants discontinued brace use within 3 months, citing a lack of perceived benefit and some discomfort [6]. Further, in vivo measurements of joint space [7-9] and joint load [10] showed that bracing may not reduce medial loads for some subjects. To interpret these inconsistent results and improve future brace designs, we must improve our understanding of the biomechanical factors that contribute to brace effectiveness.

There are three primary mechanisms through which a knee brace might unload the medial condyle: direct application of an external brace abduction moment, altered gait dynamics, or modified muscle activation [11]. Previous studies have measured brace abduction moments up to $11 \mathrm{Nm}$ [12], which could theoretically decrease medial compartment loads by $11 \%$ [13]. Additionally, bracing can alter gait kinematics [14-16], kinetics [17-19], and antagonistic cocontraction of quadriceps, hamstrings, and gastrocnemii [17,20]. These parameters also vary with osteoarthritis severity [21,22], which complicates efforts to understand brace effectiveness. 
Since it is not currently feasible to measure in vivo loads in a native knee, the link between each of these observed changes and contact loads is not well-established.

One recent study showed, using high-speed radiography, that bracing increases medial joint space by approximately $10 \%$ during stance, without inducing corresponding changes in ground reaction forces (GRFs) [23]. This evidence indicates that bracing decreases dynamic medial cartilage loading. Yet, neither medial joint space, nor the increase induced by bracing, was constant throughout stance. It is unclear how much of this variation was due to factors such as the mechanical action of the brace, muscle coordination, or even subtle shifts in gait mechanics that were not captured by the GRF. Computational modeling provides a viable approach to probe the relative contributions of each factor to internal joint loads within the musculoskeletal system [24].

Therefore, the purpose of the current study was to quantify the relative contributions of the applied brace abduction moment, gait dynamics, and muscle activation to reduction of medial loads during gait, using a detailed musculoskeletal model. We hypothesized that bracing would reduce medial knee loads not simply by applying an external brace moment, but also by inducing changes in both gait dynamics and neuromuscular control. Further, we expected to see differences in the neuromuscular response between asymptomatic and osteoarthritis groups. A secondary aim was to investigate differences in these mechanisms between two different knee braces. 


\section{Methods}

\subsection{Participants}

Participants were 20-65 years old, recreationally active and able to walk a city block (400 m), not currently taking medications for any neurological, cardiovascular, or metabolic disorders, and had no lower-limb injuries or surgeries within the last year (Table 1). Seventeen participants with moderate radiographic (Kellgren-Lawrence, $\mathrm{KL}>1$ ) and symptomatic medial knee osteoarthritis, diagnosed by an orthopaedic surgeon, formed the osteoarthritis group. Osteoarthritis participants had been prescribed a knee brace, but had not used one prior to this study. Eighteen participants with no history of any lower-limb disease formed the control group. Participants completed the WOMAC questionnaire to assess knee health [25,26], and provided written, informed consent. The study was approved by the institutional ethics review board. 


\subsection{Knee Braces}

Two off-the-shelf osteoarthritis knee braces were used in this study: OA Assist and OA Adjuster

A) OA Assist

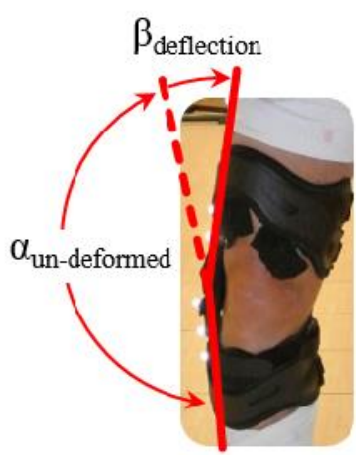

$\beta_{\text {deflection }}=\theta_{\text {measured }}-\alpha_{\text {un-deformed }}$
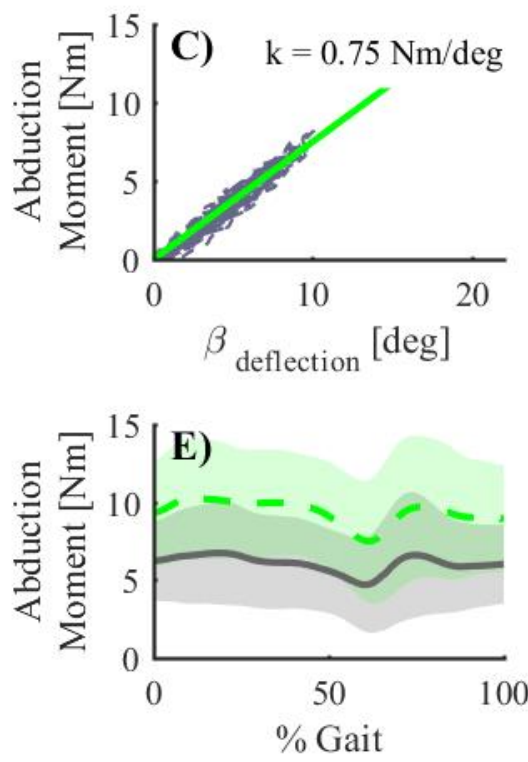

B) OA Adjuster 3
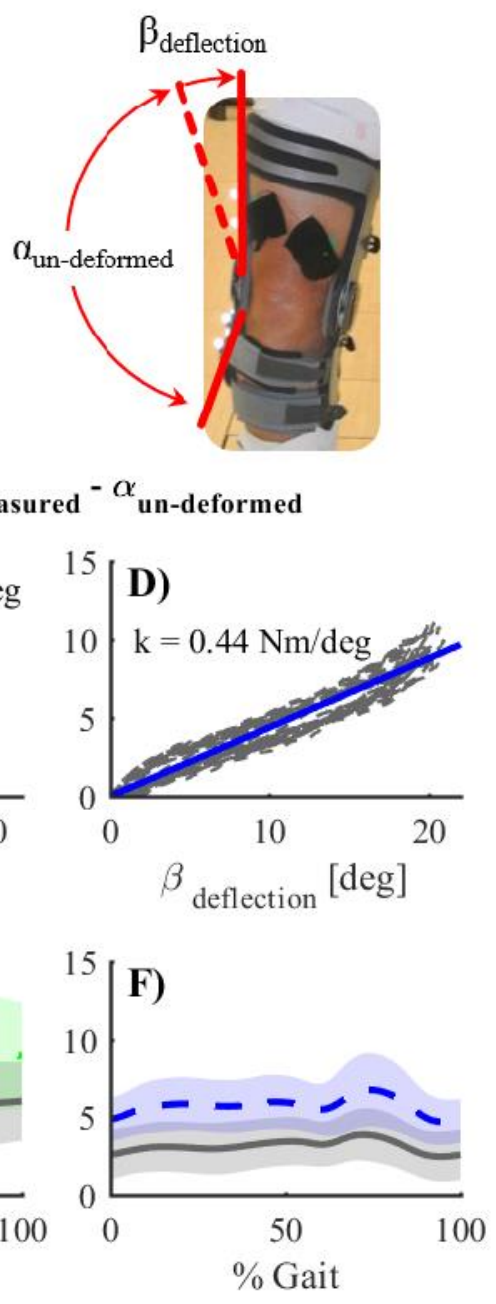

3 (DJO Global, CA, USA,

Figure 1). Both designs employ a deformable lateral beam to apply an abduction moment to the knee joint via three-point-bending [27], but exhibit substantial design differences. The OA 
Assist brace has a single lateral beam and a screw-based load adjustment mechanism (

A) OA Assist

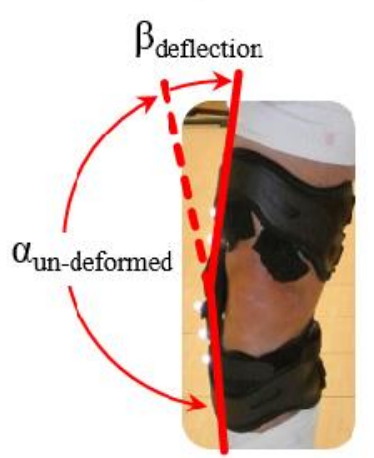

$\beta_{\text {deflection }}=\theta_{\text {measured }}-\alpha_{\text {un-deformed }}$
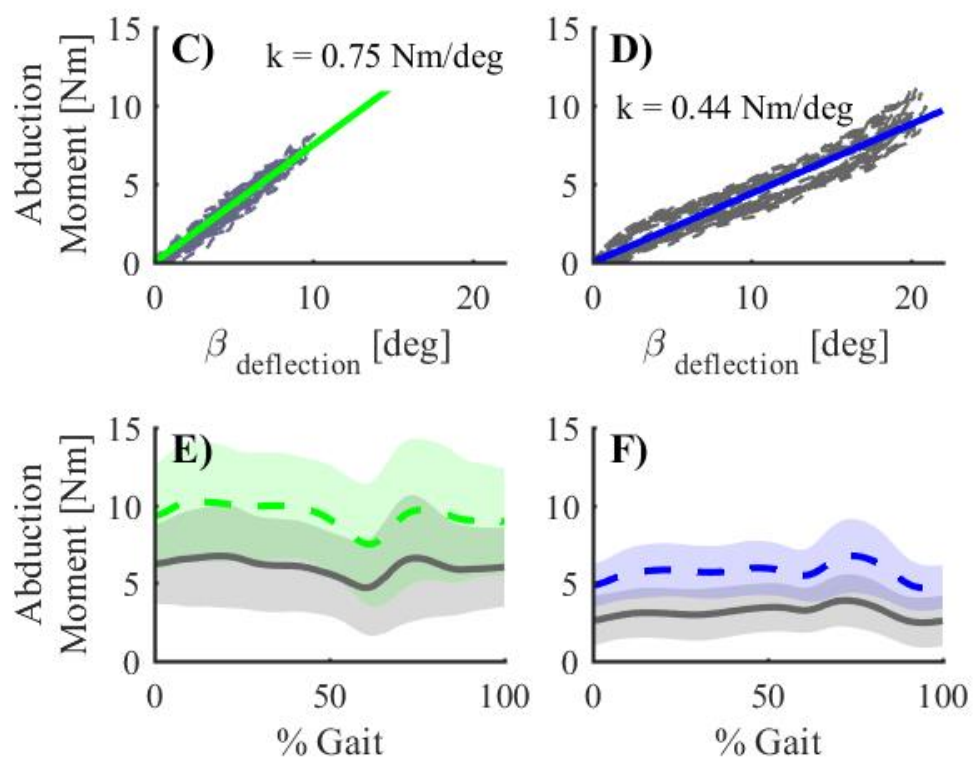
Figure 1A), while the OA Adjuster 3 brace has both medial and lateral beams and a hinge-based

A) OA Assist

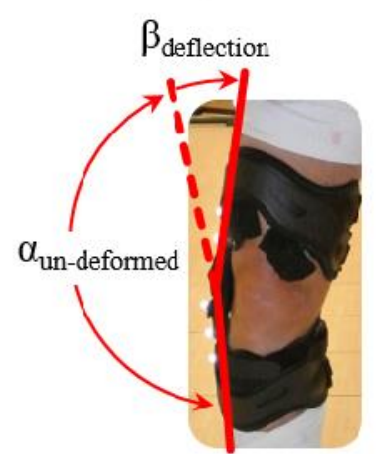

$$
\beta_{\text {deflection }}=\theta_{\text {measured }}-\alpha_{\text {un-deformed }}
$$
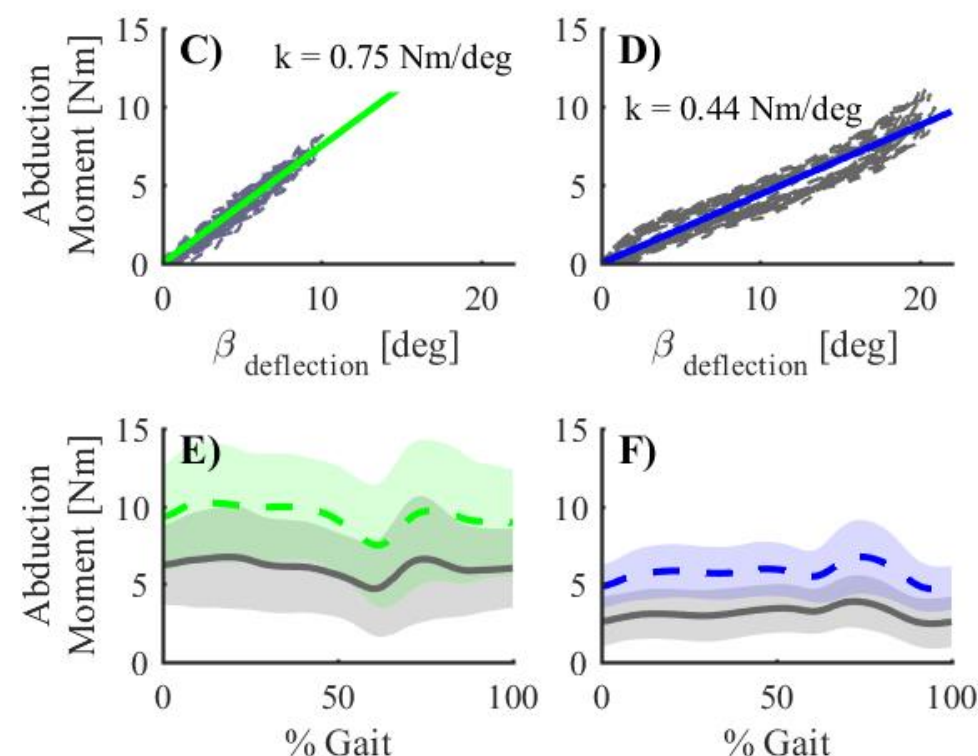

load adjustment (

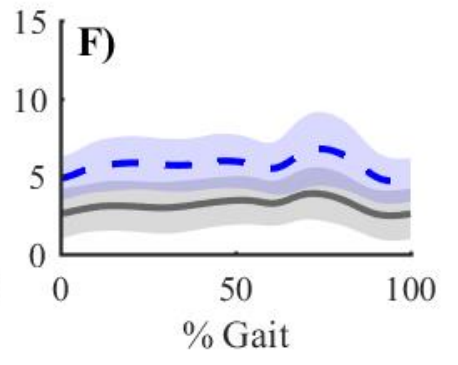

Figure 1B). Each brace was fitted per manufacturer guidelines, and the abduction load was iteratively adjusted to a maximal level that each subject perceived as tolerable for a 4-hour bout of brace usage; a previous study reported daily brace usage of $4.7 \pm 4.4$ hours [28].

\subsection{Measurements}

Eighty-one retroreflective markers were adhered to participants' limbs using elastic straps and tape [29]. Kinematics were recorded at $200 \mathrm{~Hz}$ using an eleven-camera motion capture system (Qualisys, Sweden) while ground reaction forces were measured synchronously at $1000 \mathrm{~Hz}$ using 
six tandem force platforms (AMTI, MA, USA). Participants wore their own shoes. Eight retroreflective markers were affixed to the lateral brace beam to compute brace adduction
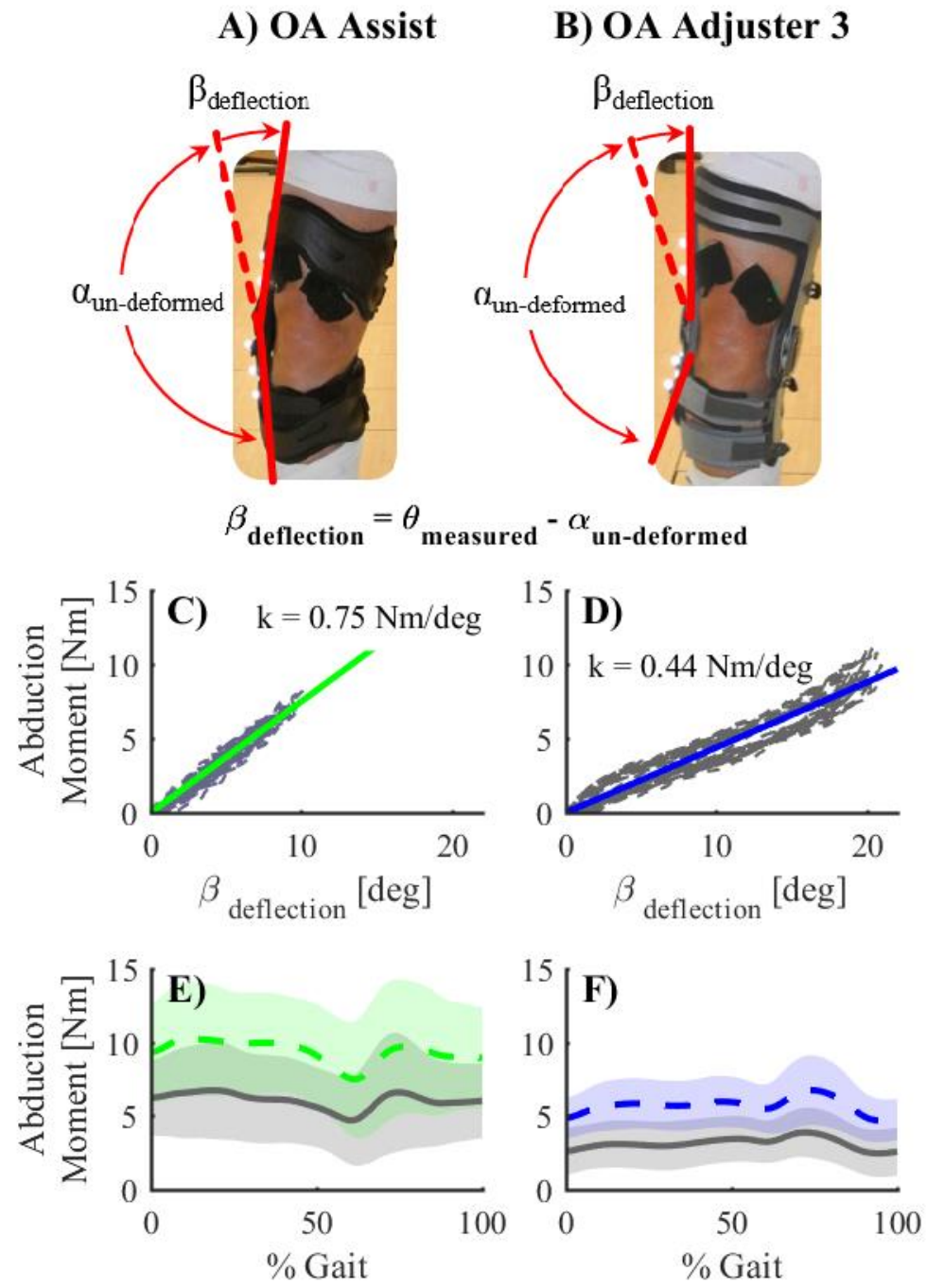

deflection angles ( 
Figure 1A,B), which were multiplied by calibrated brace stiffness (

A) OA Assist

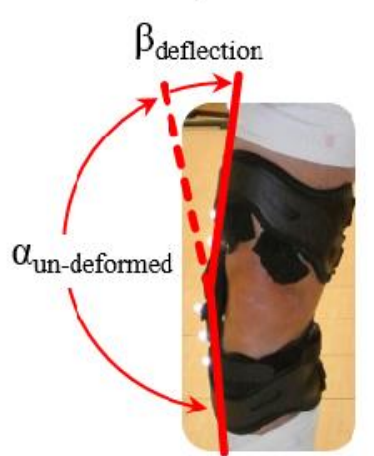

$\beta_{\text {deflection }}=\theta_{\text {measured }}-\alpha_{\text {un-deformed }}$
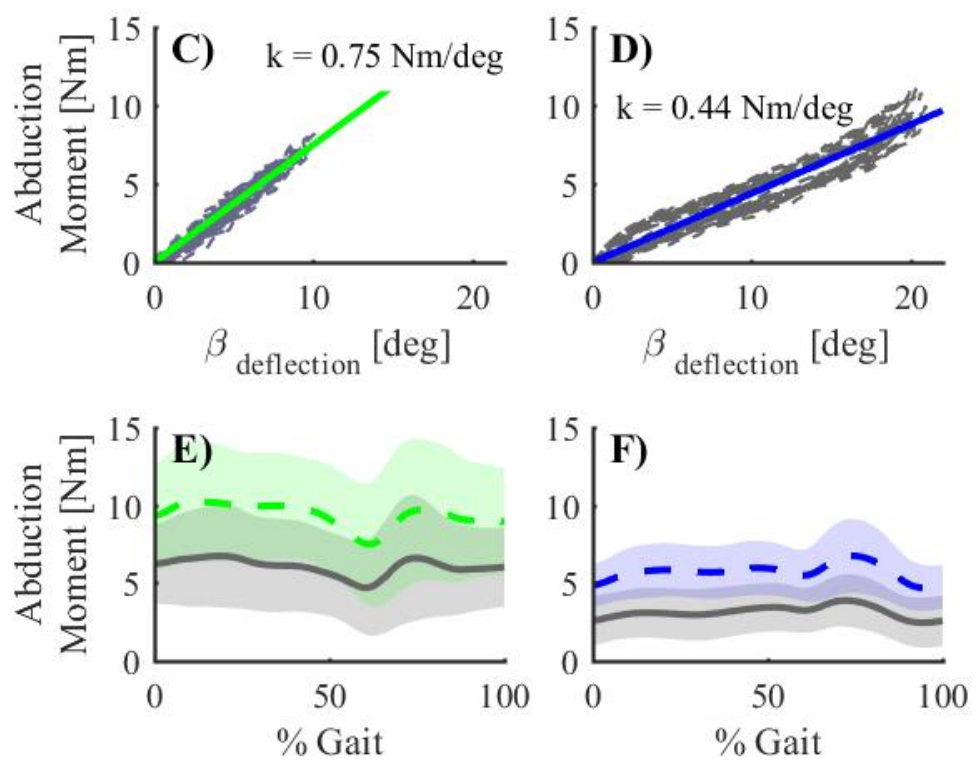
Figure 1C,D) to yield brace abduction moments (
A) OA Assist
B) OA Adjuster 3
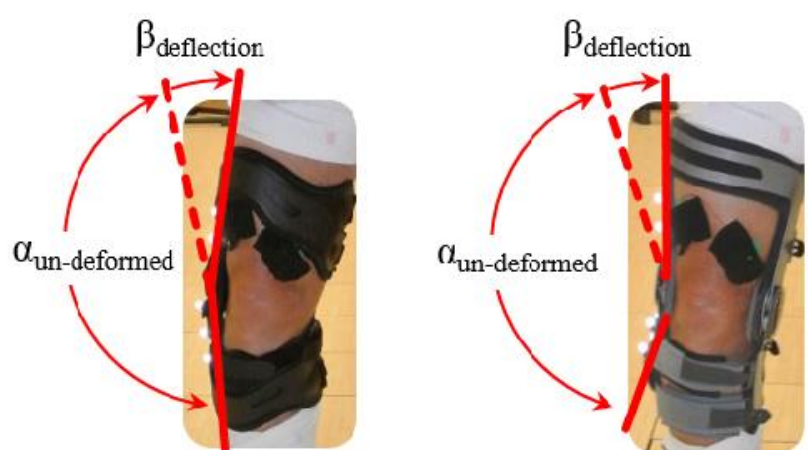

$\beta_{\text {deflection }}=\theta_{\text {measured }}{ }^{-\alpha}$ un-deformed
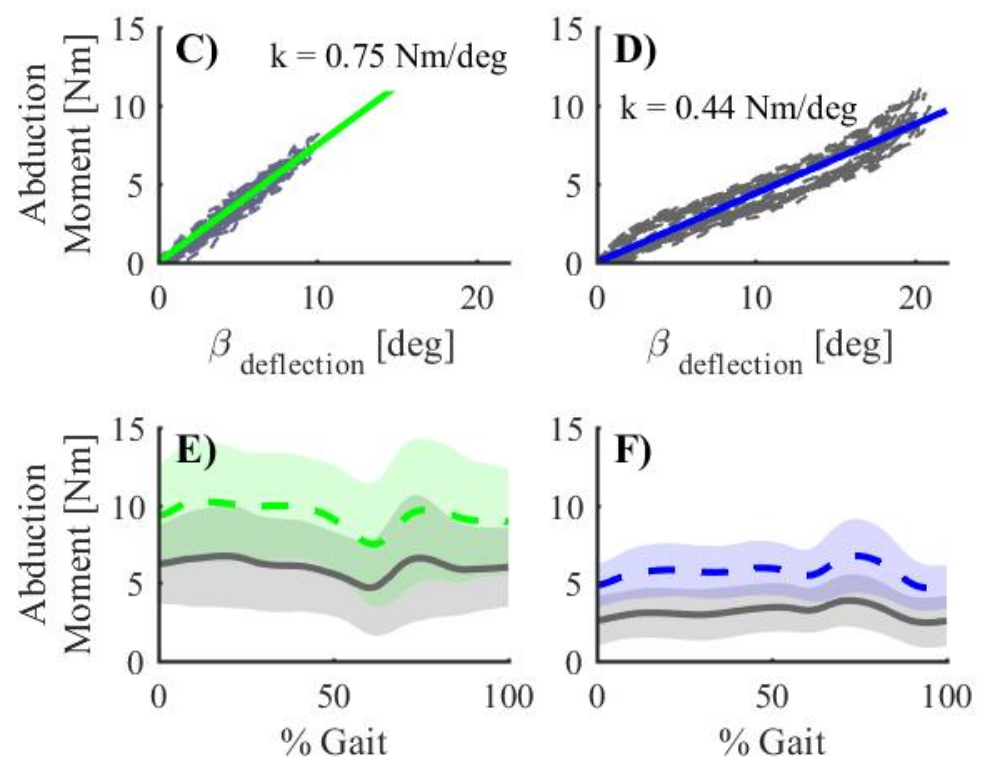

Figure 1E,F) [30].

Surface electromyograms (EMG) (Trigno, Delsys Inc, MA, USA) were recorded at $1000 \mathrm{~Hz}$ from twelve muscles on the braced leg: rectus femoris, vastus medialis, vastus lateralis, biceps femoris, semitendinosus, medial gastrocnemius, lateral gastrocnemius, tibialis anterior, soleus, gluteus maximus, gluteus medius, and tensor fascia lata. Electrodes were located per Seniam 
guidelines (www.seniam.org), except for vastus lateralis and vastus medialis. Due to geometric

A) OA Assist

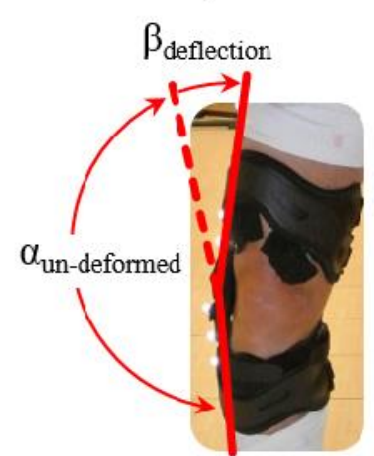

$\beta_{\text {deflection }}=\theta_{\text {meas }}$
B) OA Adjuster 3

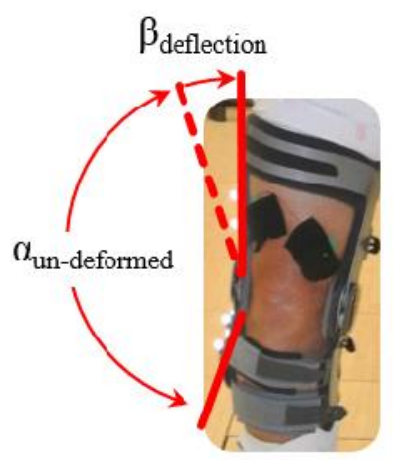

easured $^{-\alpha}$ un-deformed
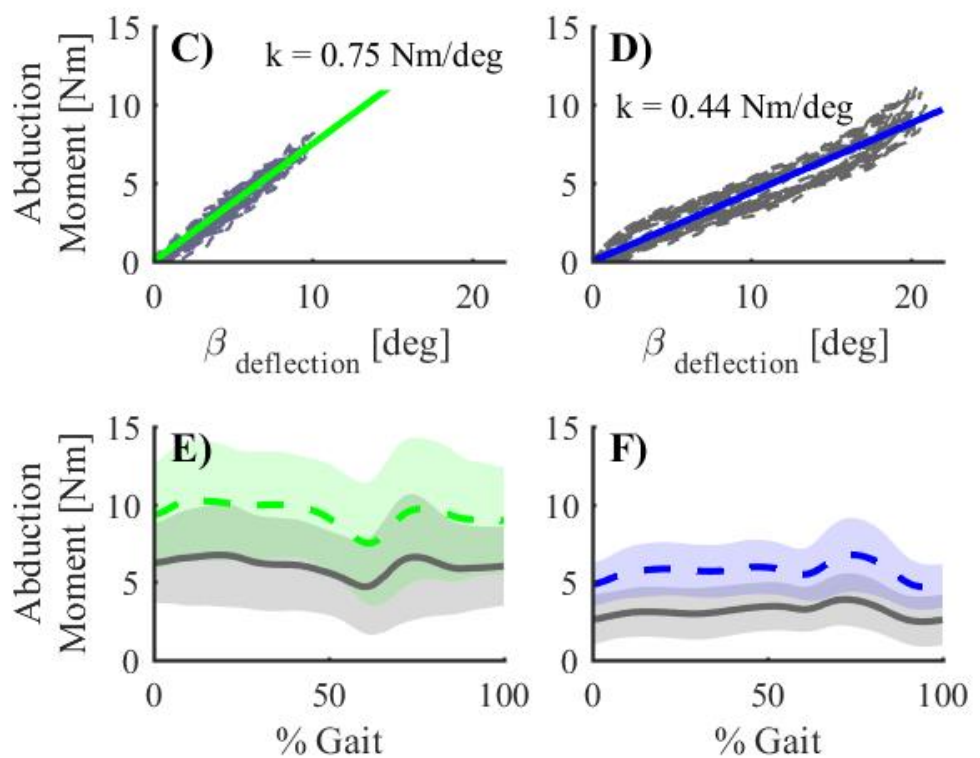

interference with the brace (

Figure 1), electrodes for these muscles were placed distal to the brace strap, but as far as possible from the knee joint to avoid regions of muscle innervation [31] and large skin motion artefact [32].

\subsection{Procedure}

Participants performed two thirty-second treadmill gait trials (not reported) and eight overground gait trials at self-selected speed in each of three conditions in the following order: unbraced, wearing an OA Adjuster 3 brace, and wearing an OA Assist brace. The OA Assist brace was 
always tested last due to its greatest potential interference with EMG sensors (

A) OA Assist

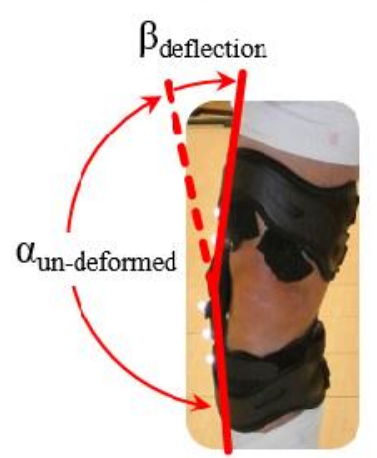

$\beta_{\text {deflection }}=\theta_{\text {meas }}$
B) OA Adjuster 3

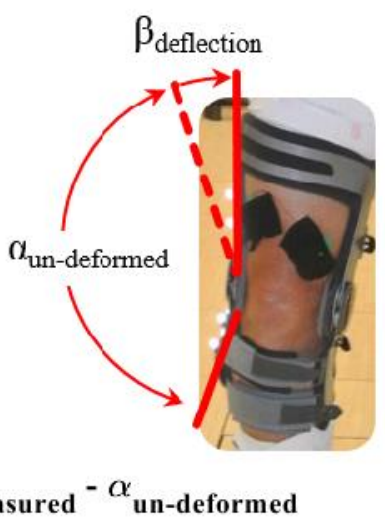

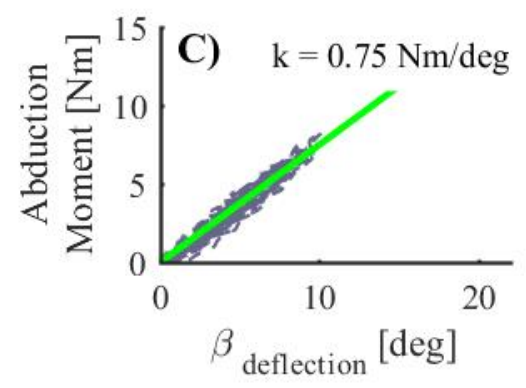
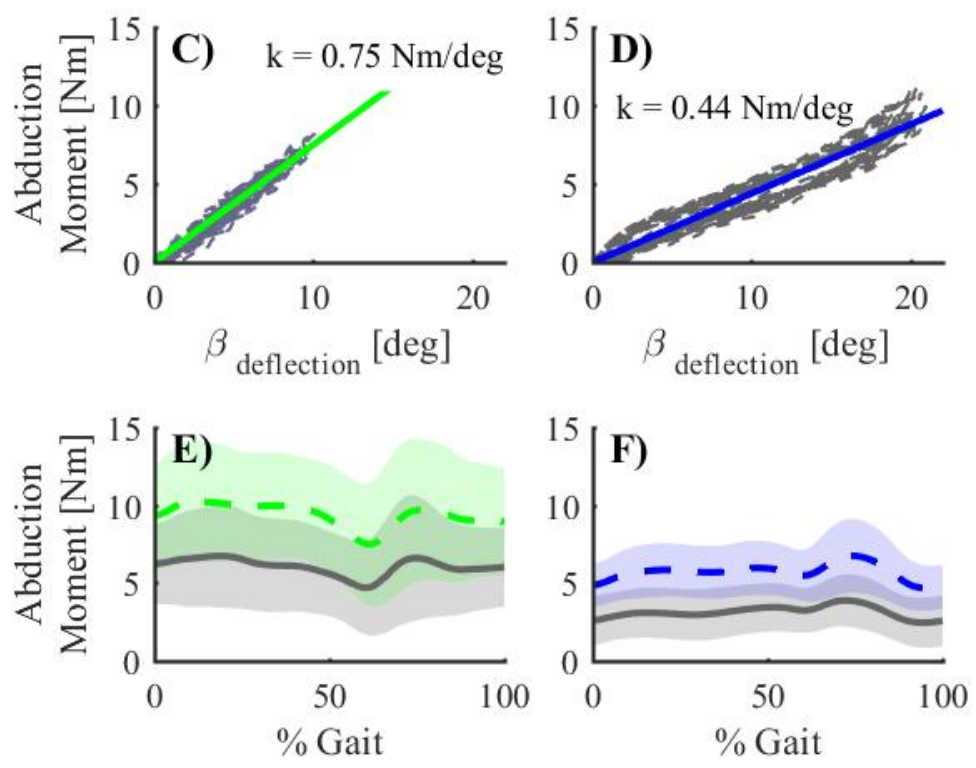

Figure 1A,B).

Subsequently, participants performed maximum voluntary isometric contractions at the ankle, hip, and knee using a dynamometer (Biodex Medical Systems, NY, USA). Exercises included: seated knee flexion and extension with knee flexed $90^{\circ}$; ankle plantar- and dorsi-flexion with neutral ankle and knee flexed $100^{\circ}$; supine hip extension against hip restraining belt with the hip flexed $30^{\circ}$; and hip abduction with the hip abducted $20^{\circ}$. For each exercise, participants performed two, three-second contractions with verbal encouragement, followed by 30 seconds of 
rest [33]. Finally, participants obtained standing full-length radiographs to measure frontal plane knee alignment [34], medial knee joint space [35], limb length, and pelvis width.

\subsection{Data Processing}

Marker trajectories and ground reaction forces were low-pass filtered at $6 \mathrm{~Hz}$ and $12 \mathrm{~Hz}$, respectively using a second order zero lag butterworth filter [36] in MATLAB (R2012b, The MathWorks, MA, USA). Electromyogram data were band-pass filtered (20-450Hz), rectified, enveloped using a $6 \mathrm{~Hz}$ low-pass butterworth filter [37], and normalized to the maximum observed signal across all gait and isometric trials [33,38]. For each subject and brace condition, the first "clean" trial of the eight attempts was exported for musculoskeletal modeling. A “clean" trial required alternating, independent foot placement on three successive force platforms such that all contralateral and ipsilateral external ground reaction forces were measured for the stride.

\subsection{Musculoskeletal Model}

A generic musculoskeletal model with 96 muscles and 3-degree-of-freedom (DOF) hip joints, 1 DOF knee joints, and 1 DOF ankle joints [39] was modified to include subject-specific static frontal plane alignment and frontal-plane hinge joints at medial and lateral knee contact locations [40,41]. These hinge joints were locked at 0 degrees during scaling and inverse kinematics, and remained at this default position when "unlocked" (in OpenSim terminology) to enable reporting of moments and muscle moment arms at each contact location. The model was scaled to each participant using radiographic leg length, pelvis width and anatomical markers. Inverse kinematics, residual reduction (RRA), and muscle analysis were performed using OpenSim 3.2 [42]. Inverse kinematics solved an optimization problem at each time step for the generalized coordinates (joint angles and translations) that positioned the skeletal model to best-match experimental marker trajectories [42]. Residual reduction (RRA) was used to resolve net inverse 
dynamic moments or forces for each generalized coordinate, taking into account inertia and external loads. Recognizing that segment inertial properties and skeletal motion have limited accuracy [43], RRA reduced discrepancies between measured external forces and inertial forces by i) recommending an adjustment to each subject's torso centre-of-mass (COM) location and ii) making small, temporally-smooth adjustments within measurement error $(<1 \mathrm{deg}$ or $1 \mathrm{~cm}$ pelvis translation) to generalized coordinates using a proportional-derivative controller [44]. Joint angles and moments were reported along generalized coordinate axes, computed using an intrinsic rotation sequence; this corresponds with a joint coordinate reference frame [45]. Muscle Analysis was performed in OpenSim to extract muscle moment arms for each DOF. Subsequently, muscle forces were estimated in MATLAB using static optimization with an objective function (SO-1) that minimized the muscle area-weighted sum of squared of muscle stress [40], with additional weight factors of 1.5 on gastrocnemii, 2 on hamstrings and 1 on all other muscles. Weighting each muscle by its area ensures that sub-division of a muscle into multiple rope-like actuators does not inherently alter the total cost of muscle recruitment [46]. Placing additional weights on gastrocnemii and hamstrings muscles improves (i.e. reduces) prediction of in vivo knee contact loads by penalizing activation of bi-articular muscles that span the knee [47]. Muscle forces were constrained to equilibrate inverse dynamic hip flexion, hip rotation, knee flexion, and ankle plantarflexion moments [40]. Compressive medial and lateral tibiofemoral contact loads, acting along the long axis of the tibia, were estimated using a quasistatic frontal-plane moment balance [48, equation 1], which equilibrated the net inverse dynamic 
knee adduction moment, including the brace abduction moment (

A) OA Assist

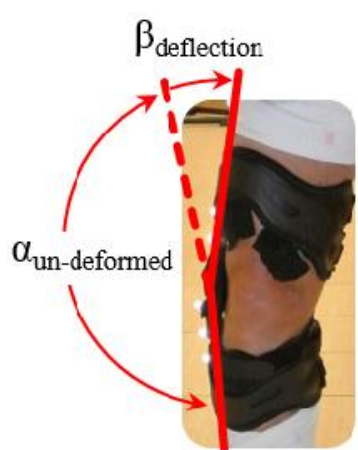

$\beta_{\text {deflection }}=\theta_{\text {measured }}-\alpha_{\text {un-deformed }}$

\section{B) OA Adjuster 3}

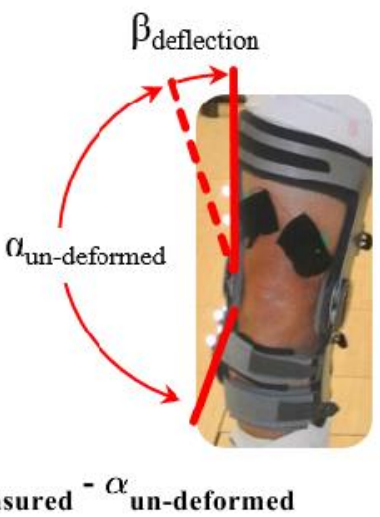

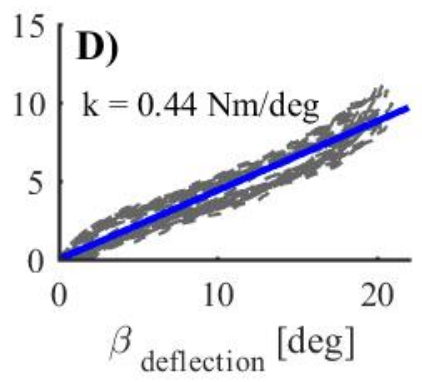
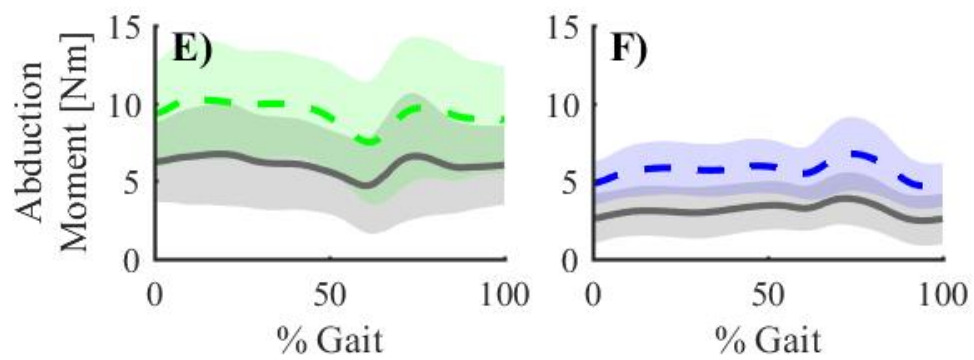

Figure 1). Finally, the individual contribution of each muscle and external load to the medial compartment force was quantified by its contribution to the moment balance equation.

To test whether errors in model-predicted muscle activations affected the interpretation of knee brace mechanics, we performed a follow-up static optimization (SO-2) based on the objective function described above (SO-1), but with additional constraints to ensure that the mean change in muscle activation from unbraced to braced conditions matched observed differences in EMG [40]. Specifically, for all muscles where significant differences were detected in EMG between 
braced and unbraced conditions, activation was prescribed in the braced conditions as the sum of the unbraced activation (SO-1) plus the mean observed change in EMG. Remaining muscles, which exhibited no change in EMG, were constrained to remain within statistically-insignificant bounds of the unbraced solution $(<2-8 \% \mathrm{MVC}$, see Supplementary Material). Thus, the constrained optimization solution (SO-2) both equilibrated the inverse dynamic joint moments and captured the neuromuscular changes that were induced by the knee braces.

\subsection{Statistical Analysis}

Discrete peak measures from joint angles, moments, contact forces, EMG, and model-predicted muscle activation (for complete list, see Supplementary Material), gait speed, and cumulative (i.e. integral thorough gait) medial, lateral, and total contact loads, were compared between brace conditions and subject groups using a two-factor ANOVA with Brace as the repeated factor, and planned Sidak post-hoc contrasts between brace conditions. Subject characteristics, and original versus follow-up static optimization solutions, were compared using unpaired t-tests.

Significance $(\alpha)$ was set at 0.05 .

\section{Results}

Osteoarthritis participants were older, heavier, walked slower, and had greater varus alignment, smaller medial joint space, and lower WOMAC scores than control subjects (Table 1). Gait speed was unaffected by bracing (Post-hoc, $\mathrm{P}>0.08$ ).

\subsection{Joint Kinematics}

Bracing increased late-stance hip extension angles $(\mathrm{P}<0.01$, 

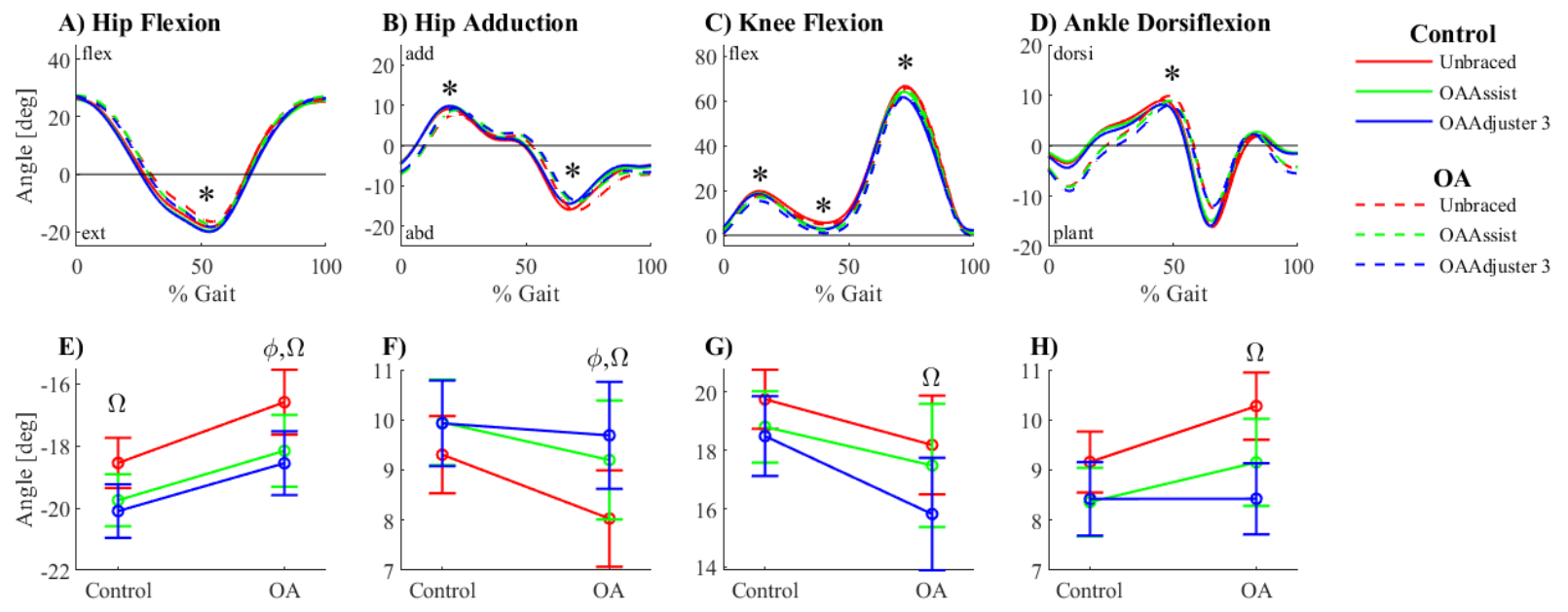

Figure 2A,E), increased early-stance hip abduction angles $(\mathrm{P}<0.01$,
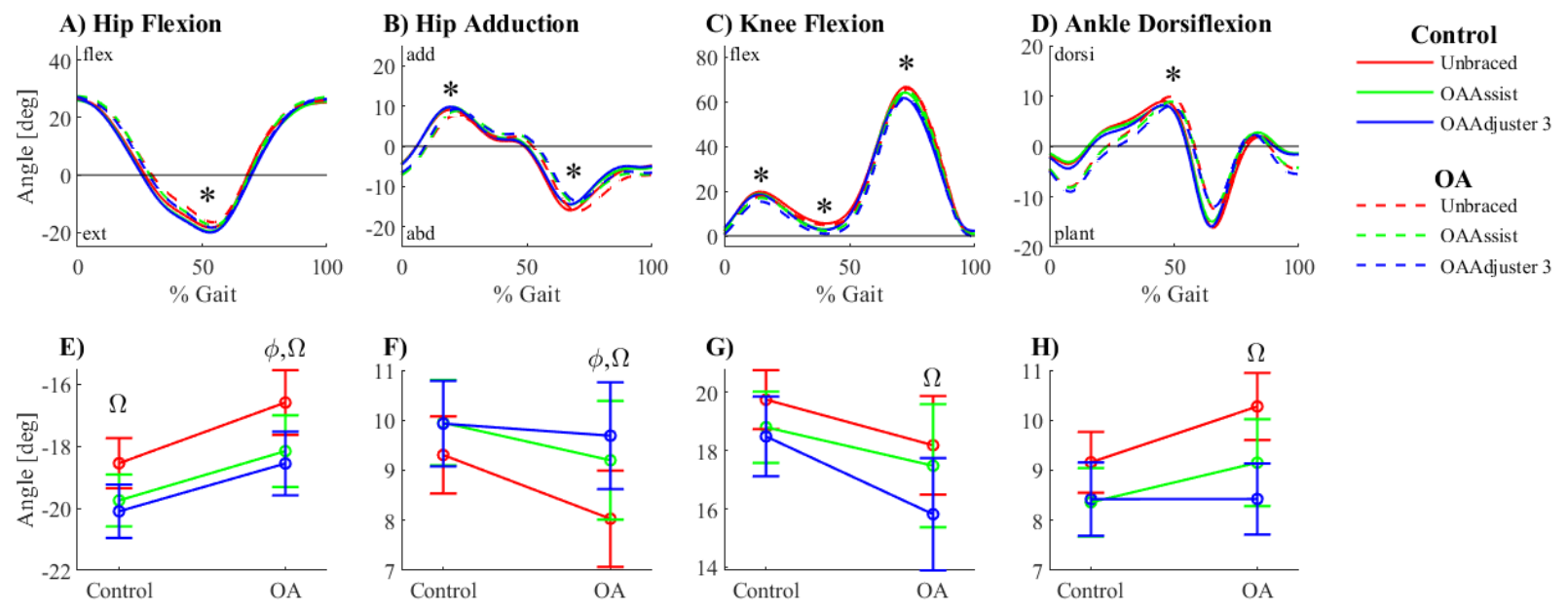

Figure 2B,F), decreased early-stance, late-stance, and swing knee flexion angles $(\mathrm{P}<0.01$, 

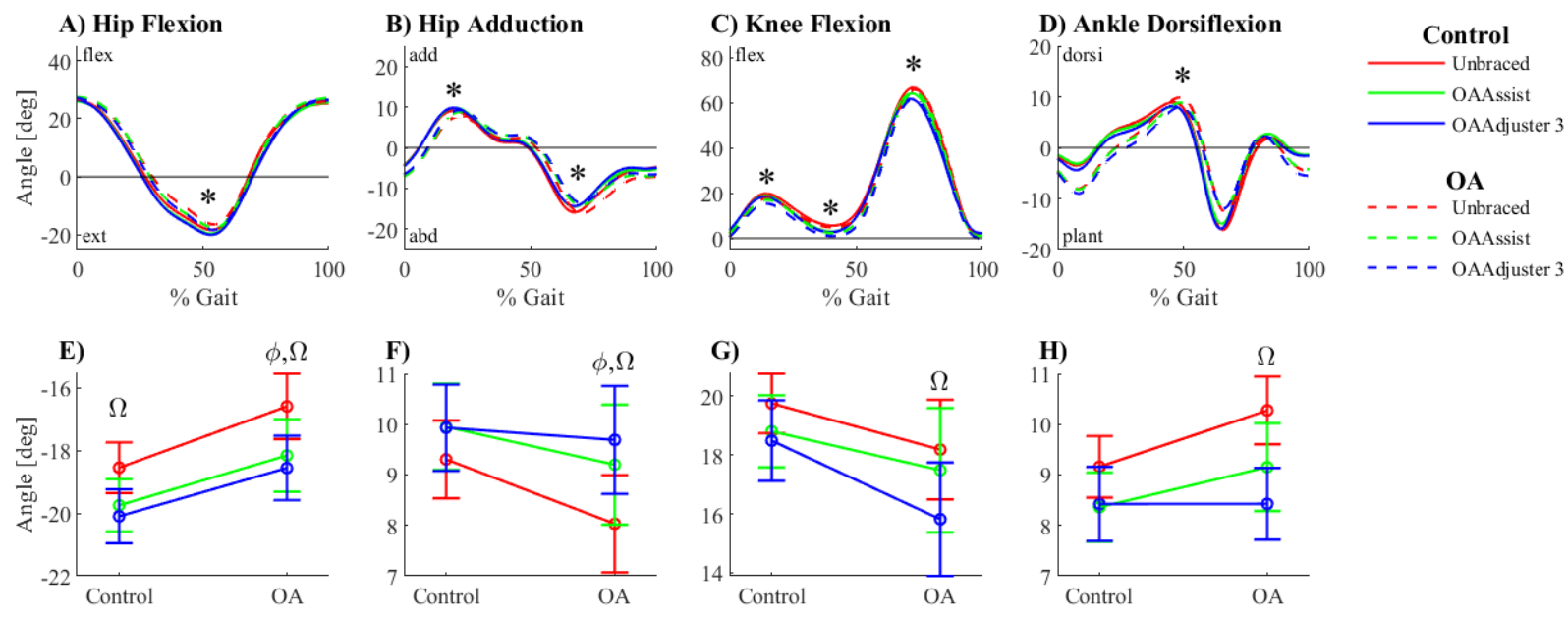

Figure 2C,G), and decreased late-stance ankle dorsiflexion angles $(\mathrm{P}<0.01$,
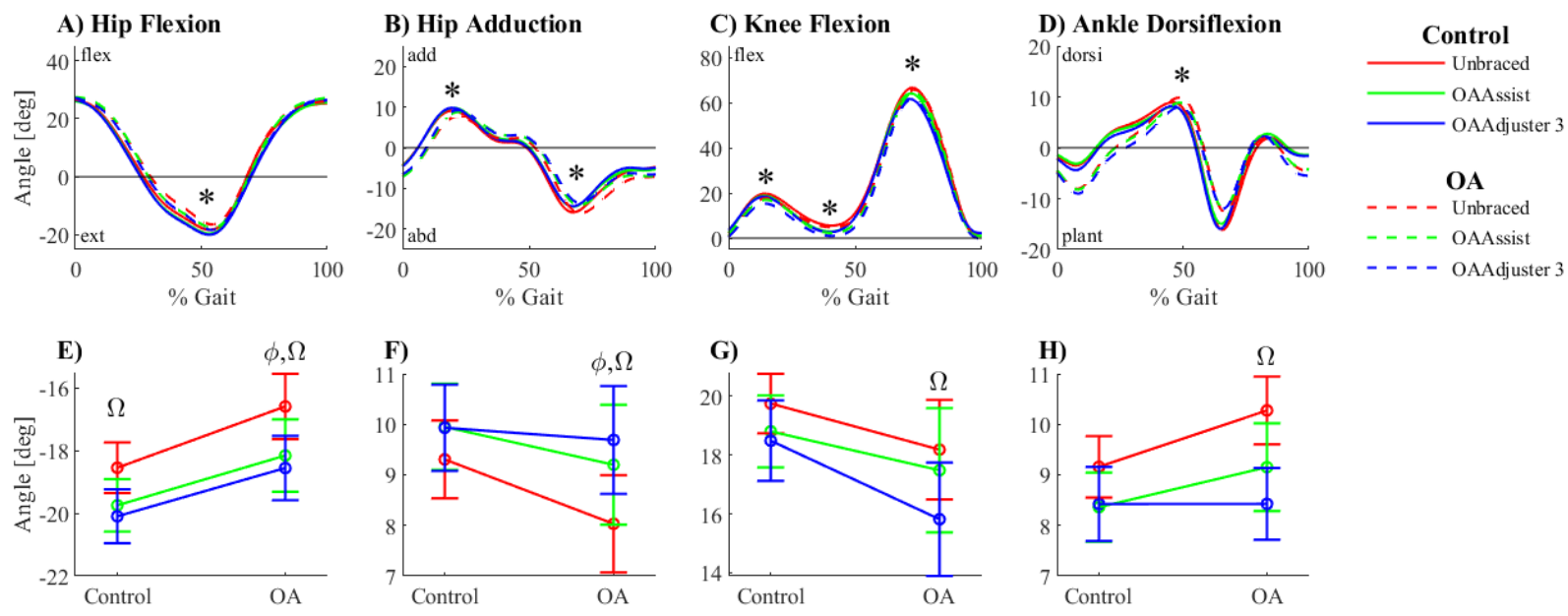

Figure 2D,H). Osteoarthritis subjects differed from control subjects only in ankle dorsiflexion (P $<0.05)$. The effect of bracing was consistent across both groups and braces. 


\subsection{Joint Moments}

Both braces decreased late-stance hip extension moments $(\mathrm{P}=0.03$,
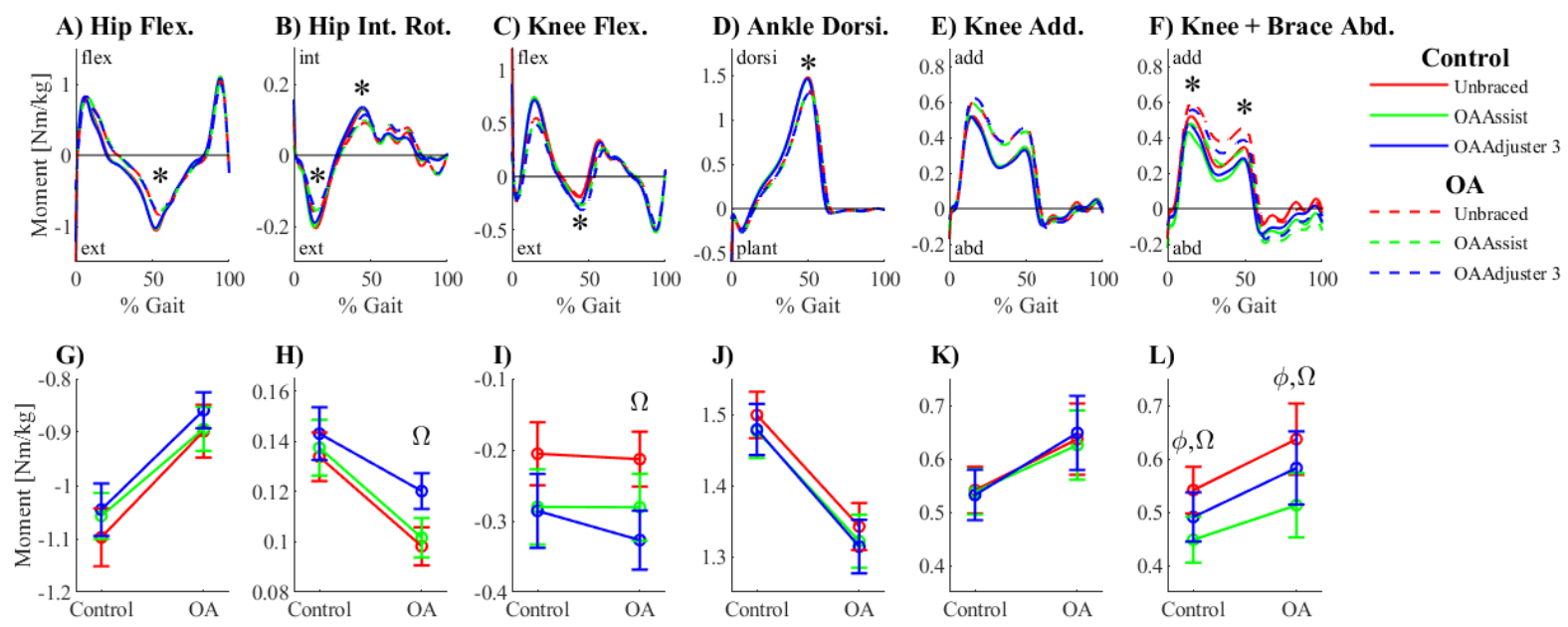

Figure 3A,G), decreased early-stance hip external rotation moments $(\mathrm{P}<0.01$,
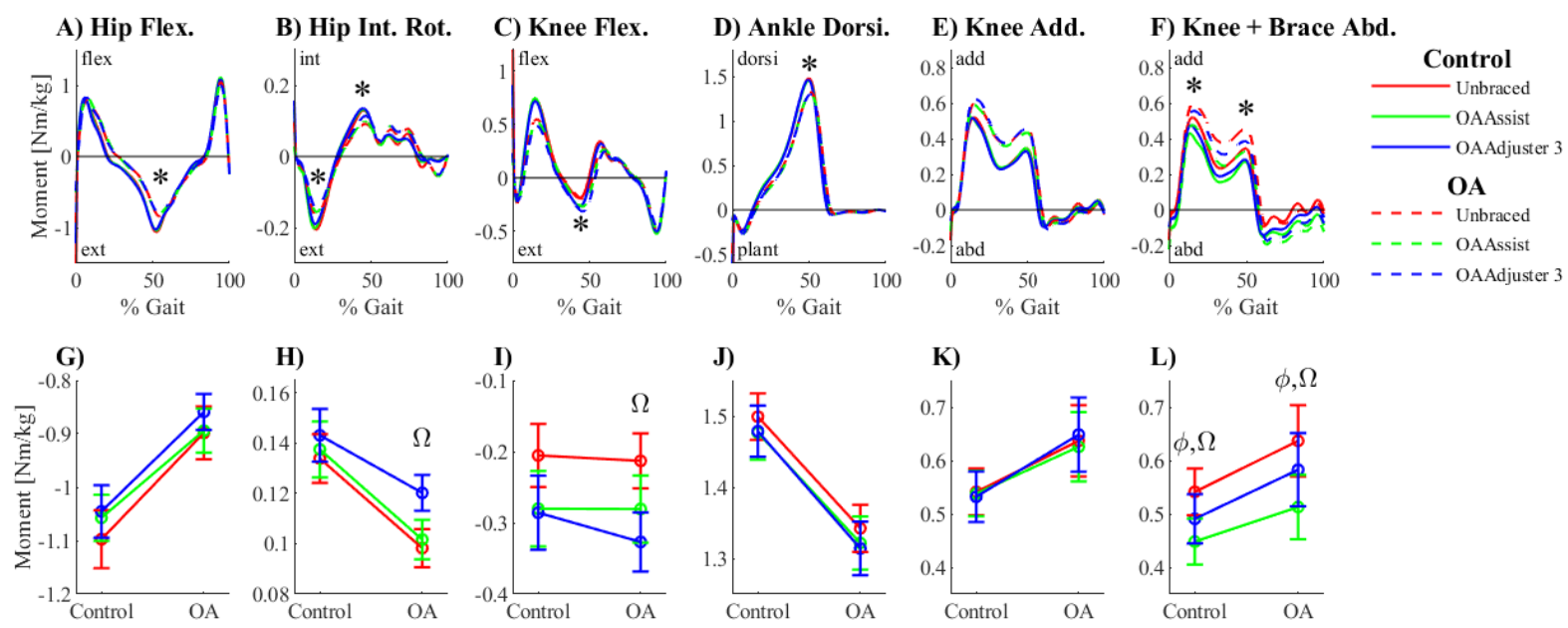

Figure 3B), increased late-stance hip internal rotation moments $(\mathrm{P}<0.01$, 

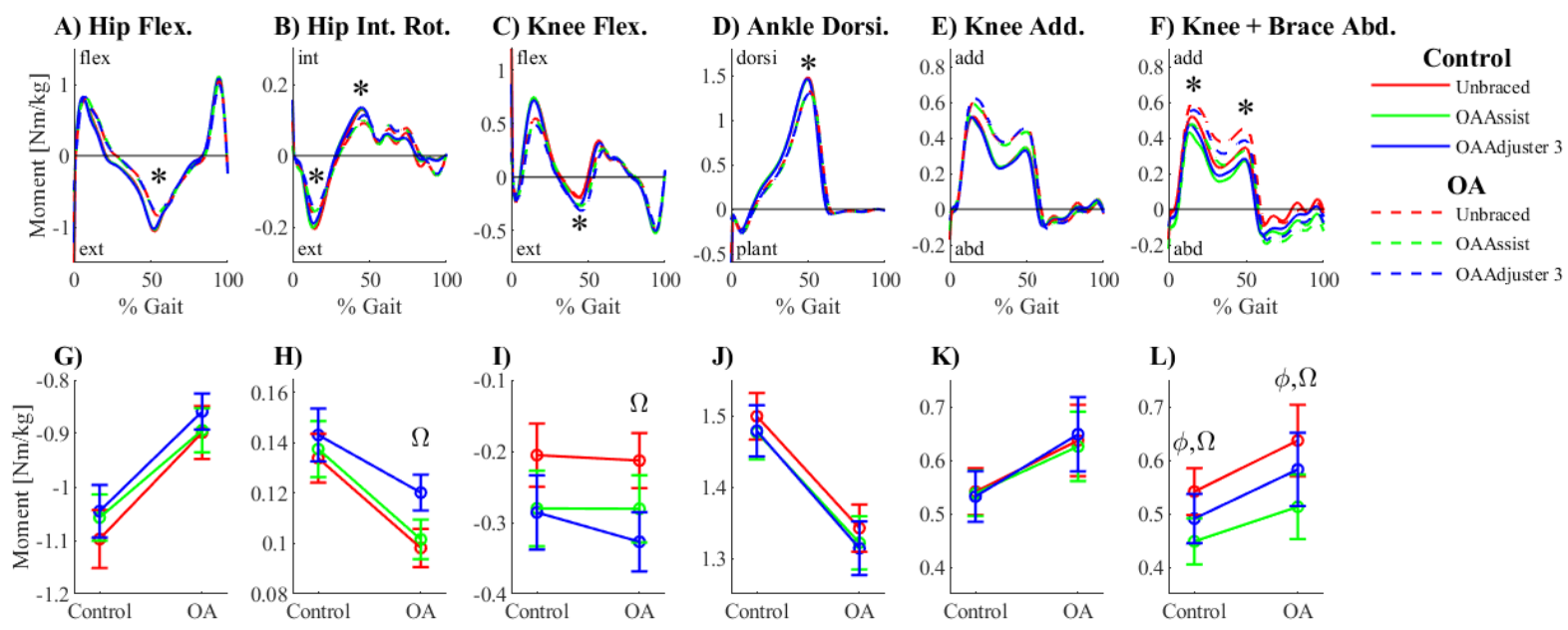

Figure 3B,H), increased late-stance knee extension moments $(\mathrm{P}<0.01$,
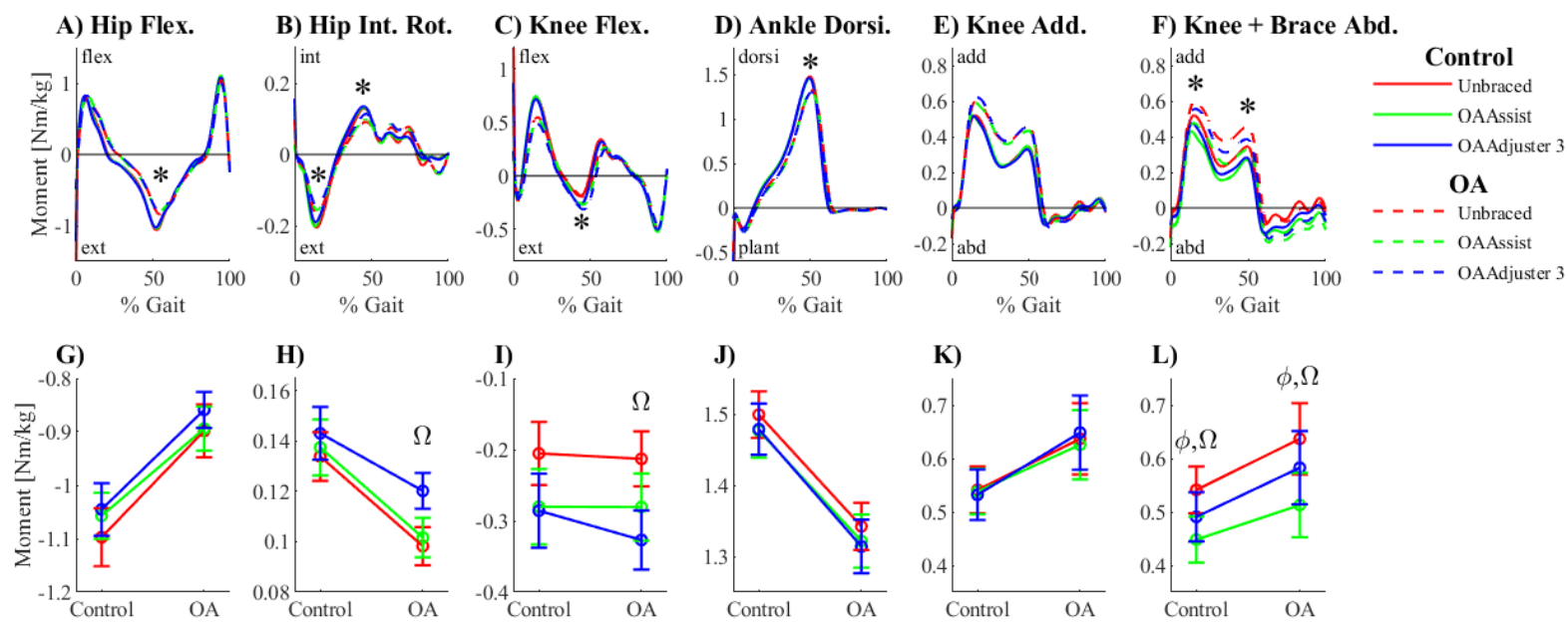

Figure 3C,I), and decreased late-stance dorsiflexion moments $(\mathrm{P}<0.01$, 

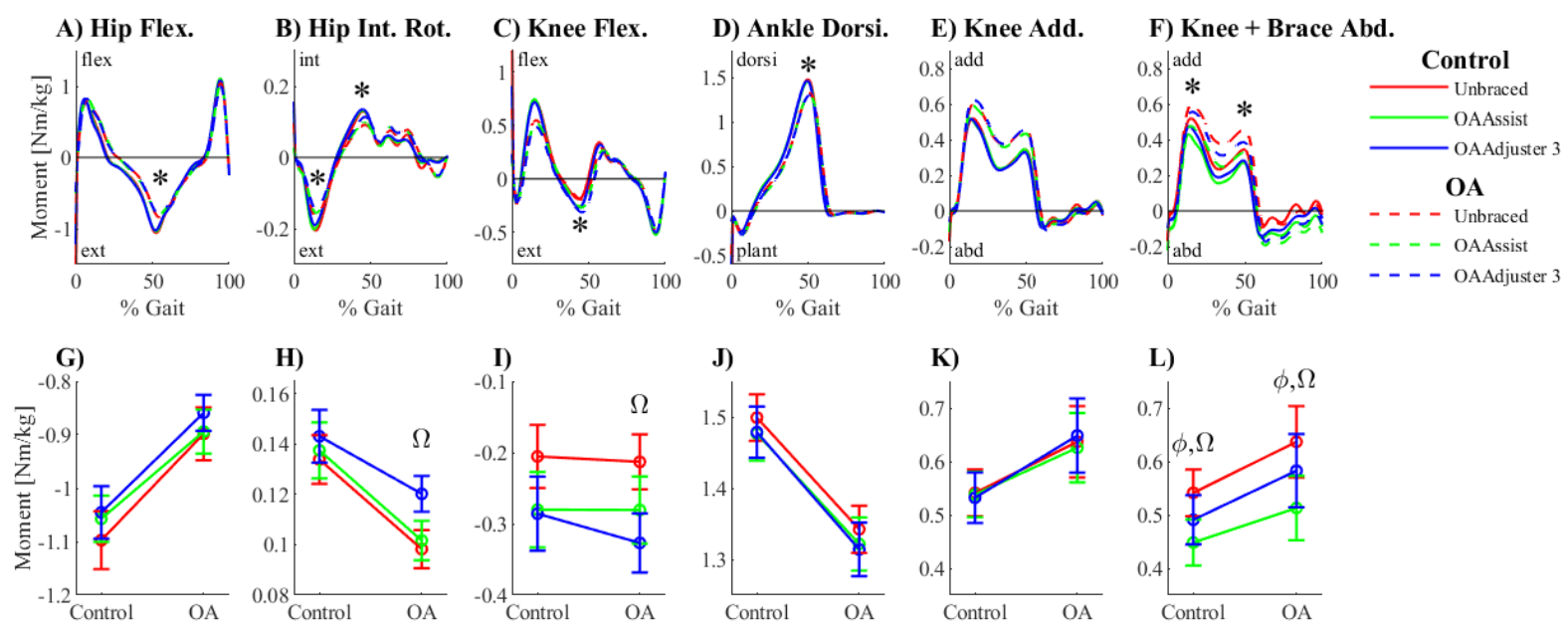

Figure 3D,J). Knee adduction moments, neglecting the brace moment, were unaffected by bracing $(\mathrm{P}>0.2$,
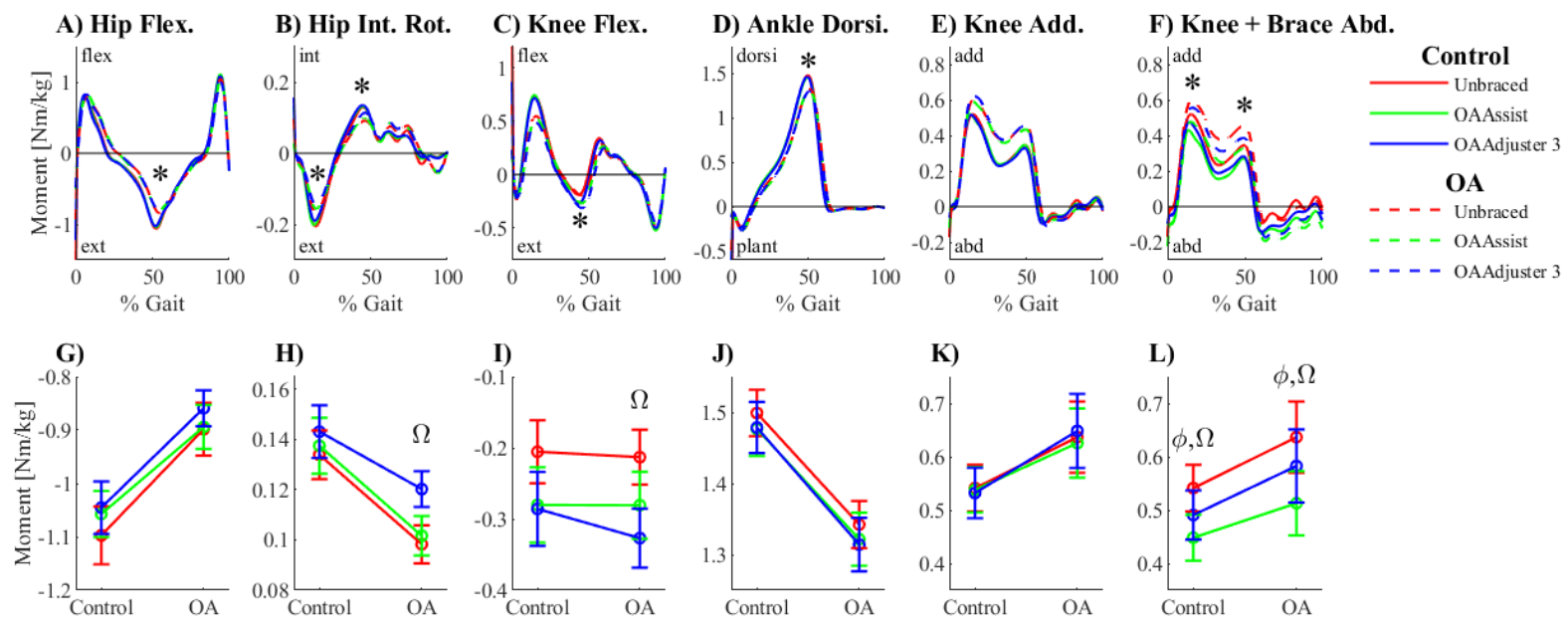

Figure 3E,K). However, net knee adduction moments, including the brace abduction moment, decreased throughout stance $(\mathrm{P}<0.01$, 

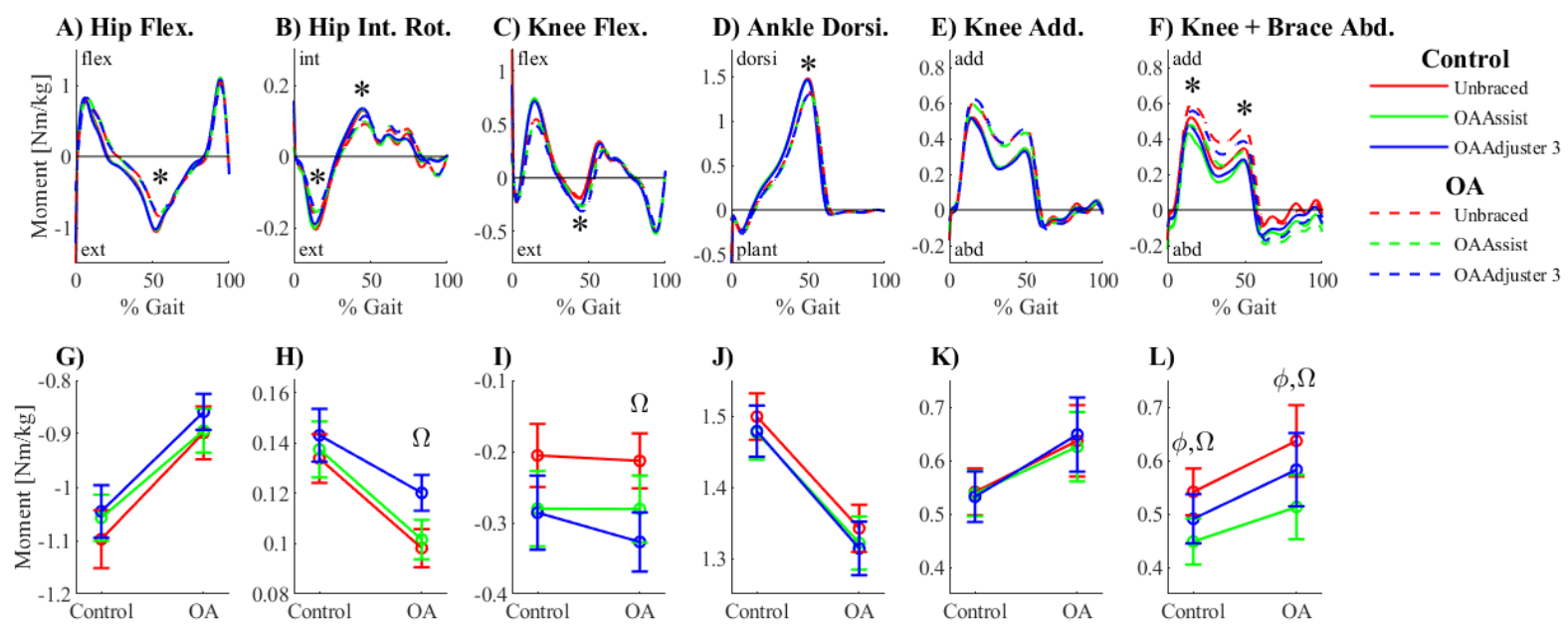

Figure 3F,L). Osteoarthritis subjects selected a larger brace abduction moment than control 
subjects for both braces, $(\mathrm{P}<0.01$,

A) OA Assist

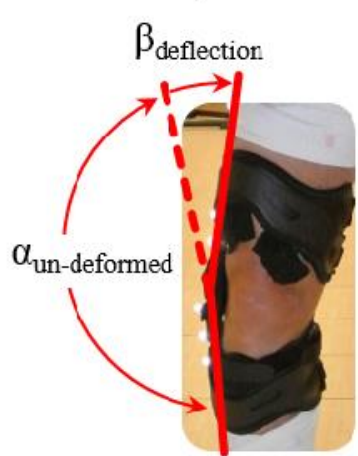

$\beta_{\text {deflection }}=\theta_{\text {measured }}-\alpha_{\text {un-deformed }}$
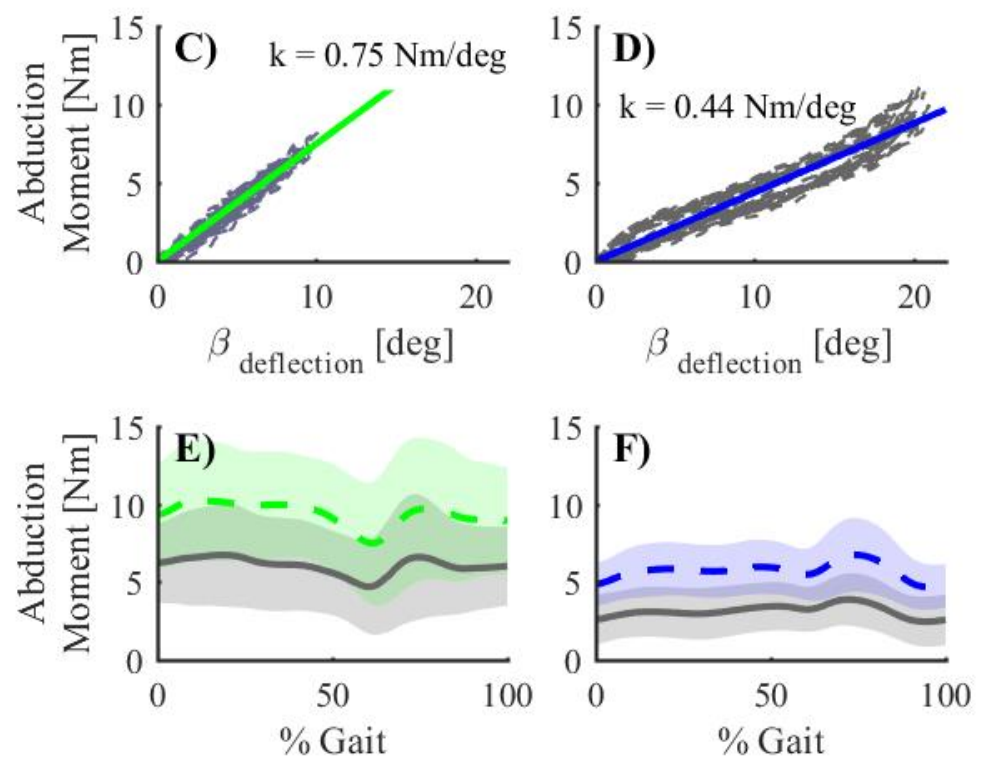
Figure 1E,F, Table 2) and both groups selected a larger brace moment with the OA Assist brace than the OA Adjuster 3 brace $(\mathrm{P}<0.01$,

\section{$\begin{array}{ll}\text { A) OA Assist } & \text { B) OA Adjuster } 3\end{array}$}
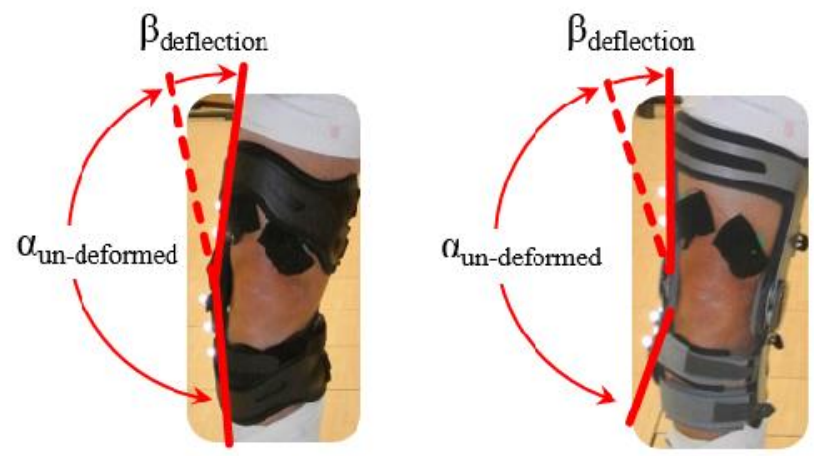

$$
\beta_{\text {deflection }}=\theta_{\text {measured }}-\alpha_{\text {un-deformed }}
$$
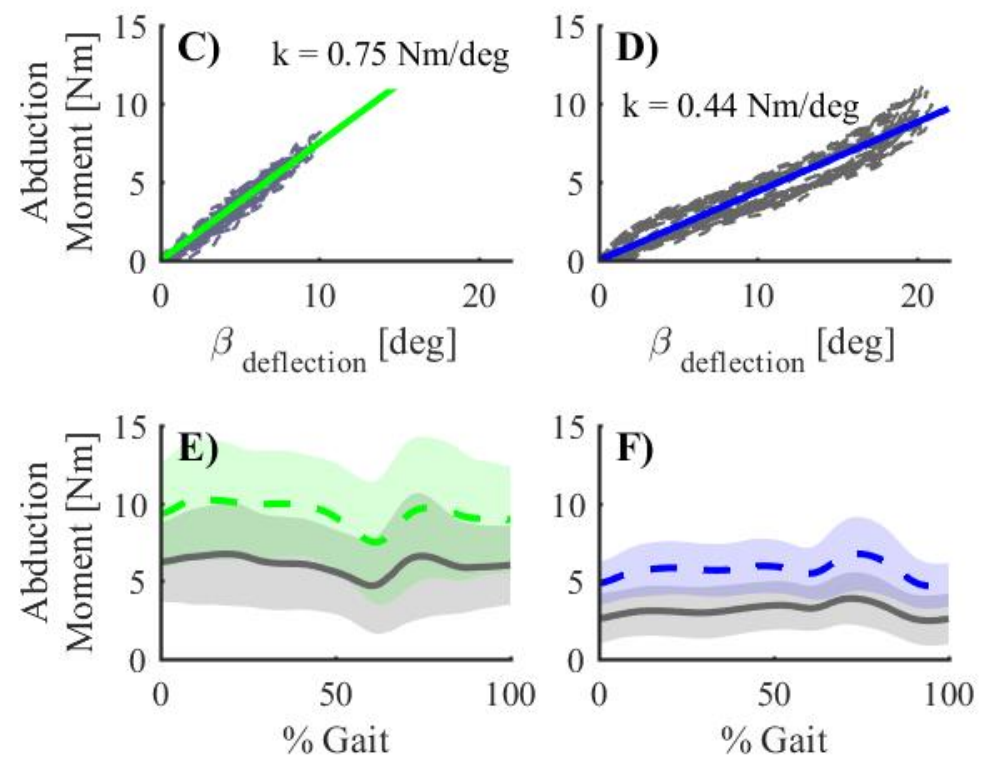

Figure 1E,F, Table 2).

\subsection{Knee Contact Forces}

Both braces shifted loading from the medial to lateral tibiofemoral compartment, with no change in the peak or cumulative total contact load ( 

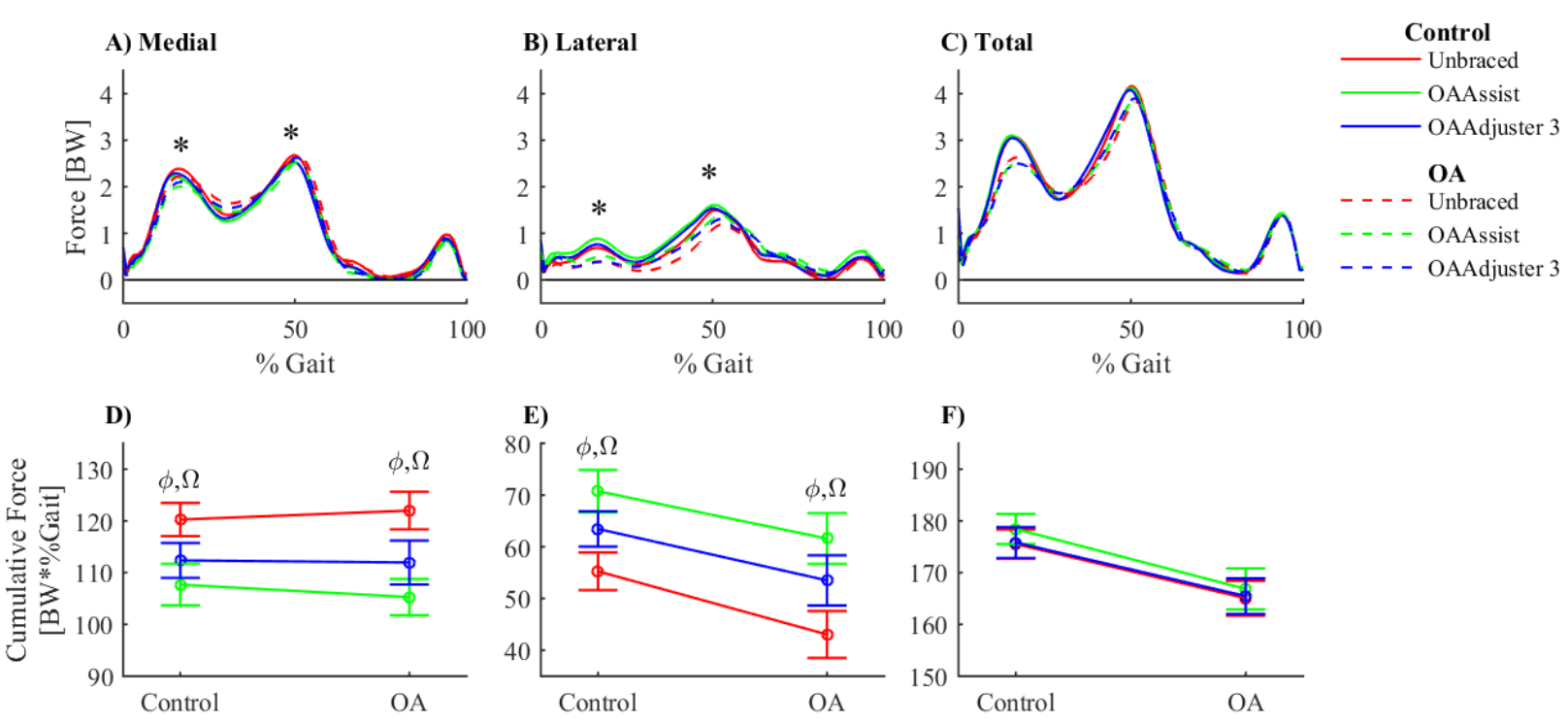

Figure 4C,F, Table 3). Early- and late-stance peak medial contact loads were reduced by 0.1 to $0.3 \mathrm{BW}$, or roughly $10 \%(\mathrm{P}<0.01$, Table 3 ,
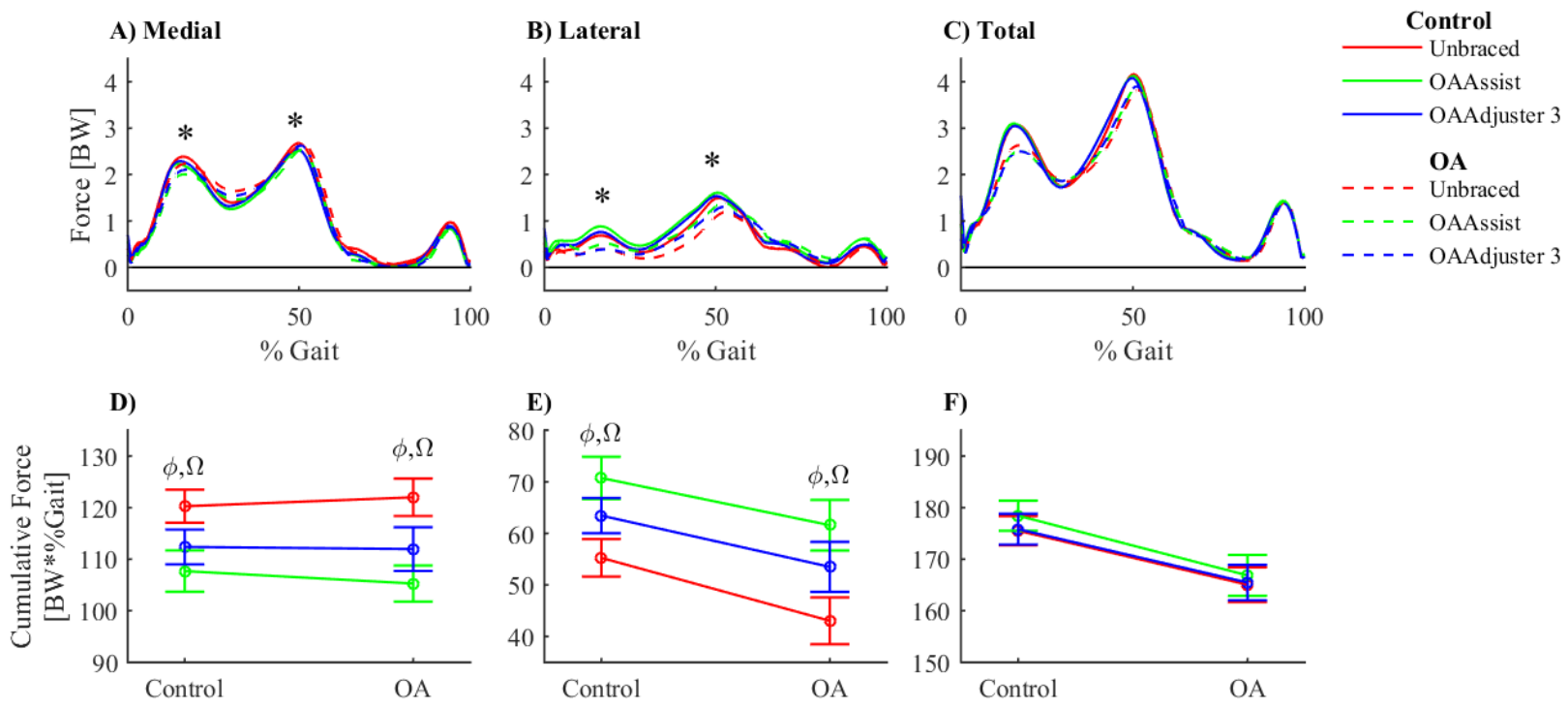

Figure 4A), with no difference between braces $(\mathrm{P}>0.37)$ or between subject groups $(\mathrm{P}>0.74$,

Table 3). Conversely, bracing increased early- and late-stance peak lateral contact loads by 0.03 to $0.2 \mathrm{BW}$, $(\mathrm{P}<0.01$, Table 3, 

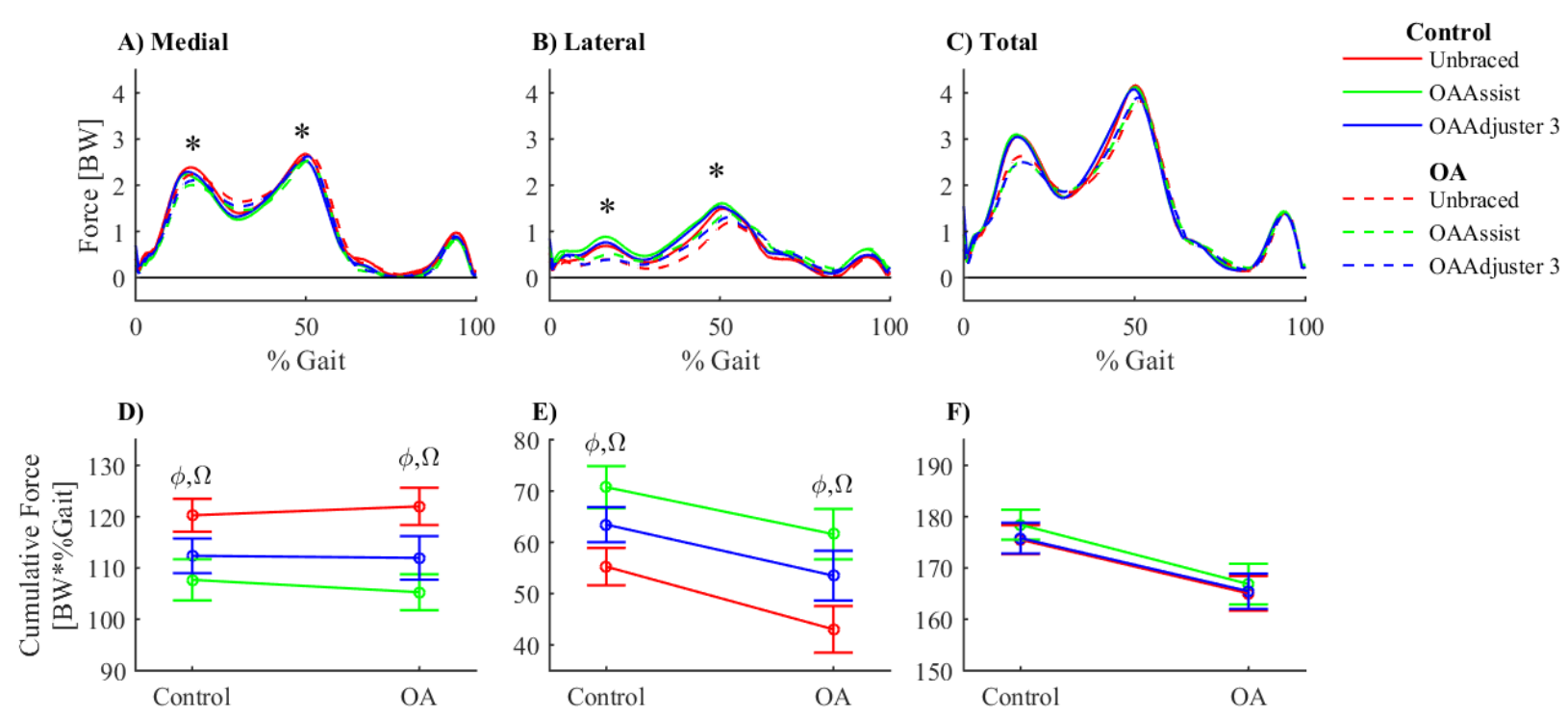

Figure 4B). The load shift was most apparent from the cumulative load (integral) throughout gait: medial loads decreased by $8-17 \mathrm{BW} * \%$ Gait, while lateral loads increased by a nearly-equal magnitude (8-19 BW*\%Gait, Table 3,
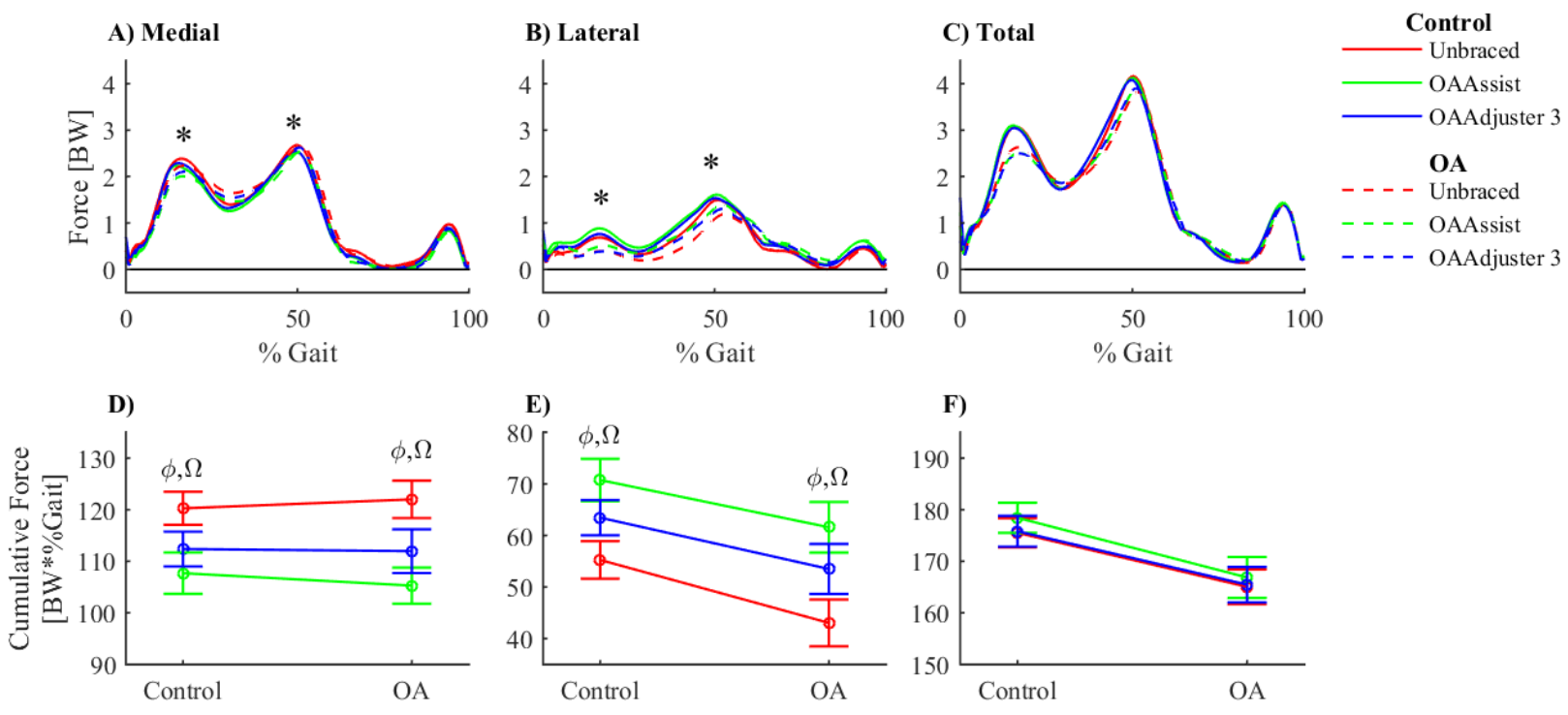

Figure 4D,E), with no difference between control and osteoarthritis groups. The cumulative medial load was reduced more for the OA Assist than the OA Adjuster 3 brace (between braces: $\mathrm{P}<0.01 \mathrm{OA}, \mathrm{P}=0.08$ Control, 

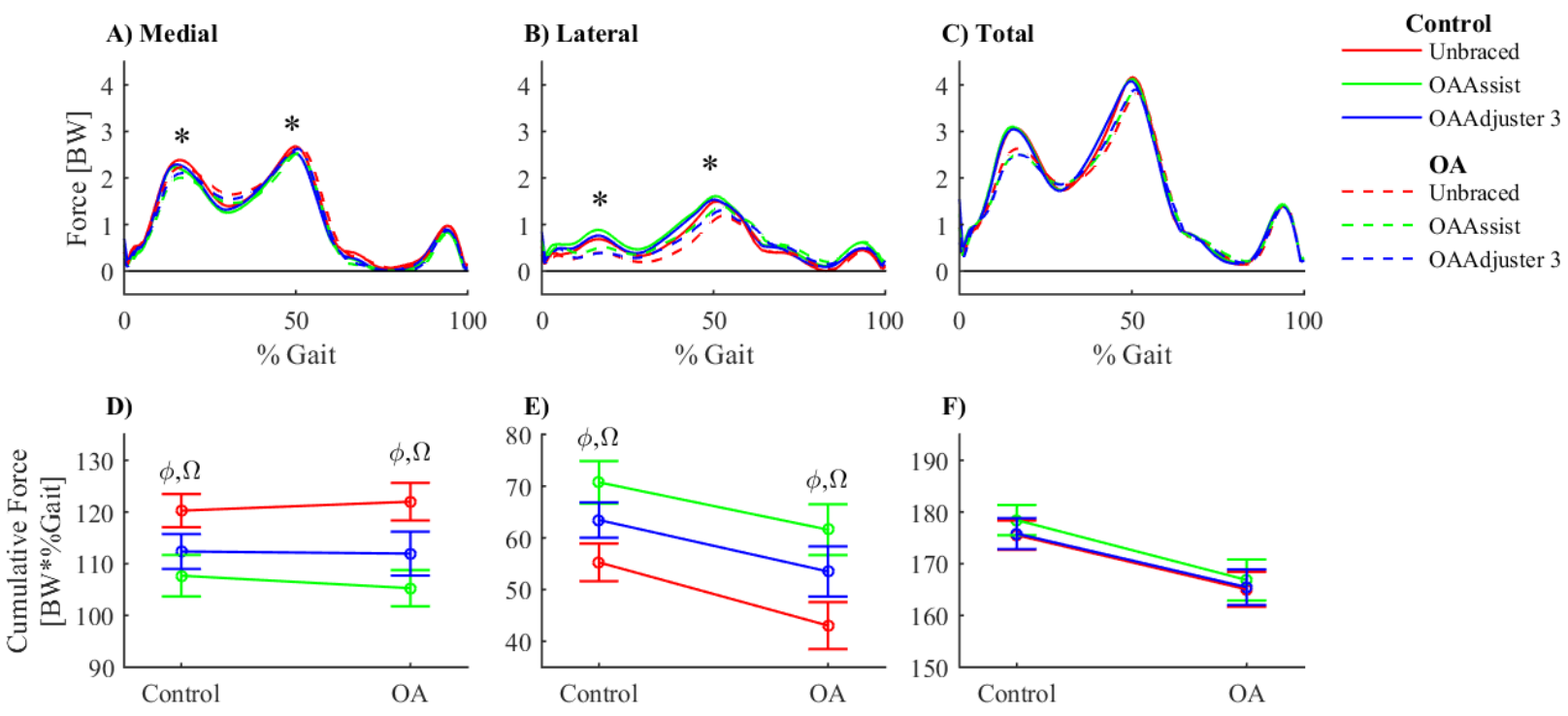

Figure 4D, Table 3).

\subsection{Muscle Activation and EMG}

The magnitude and timing of predicted muscle activations agreed well with experimental EMG (Supplementary Material). EMG was significantly affected by bracing for one muscle (biceps femoris,
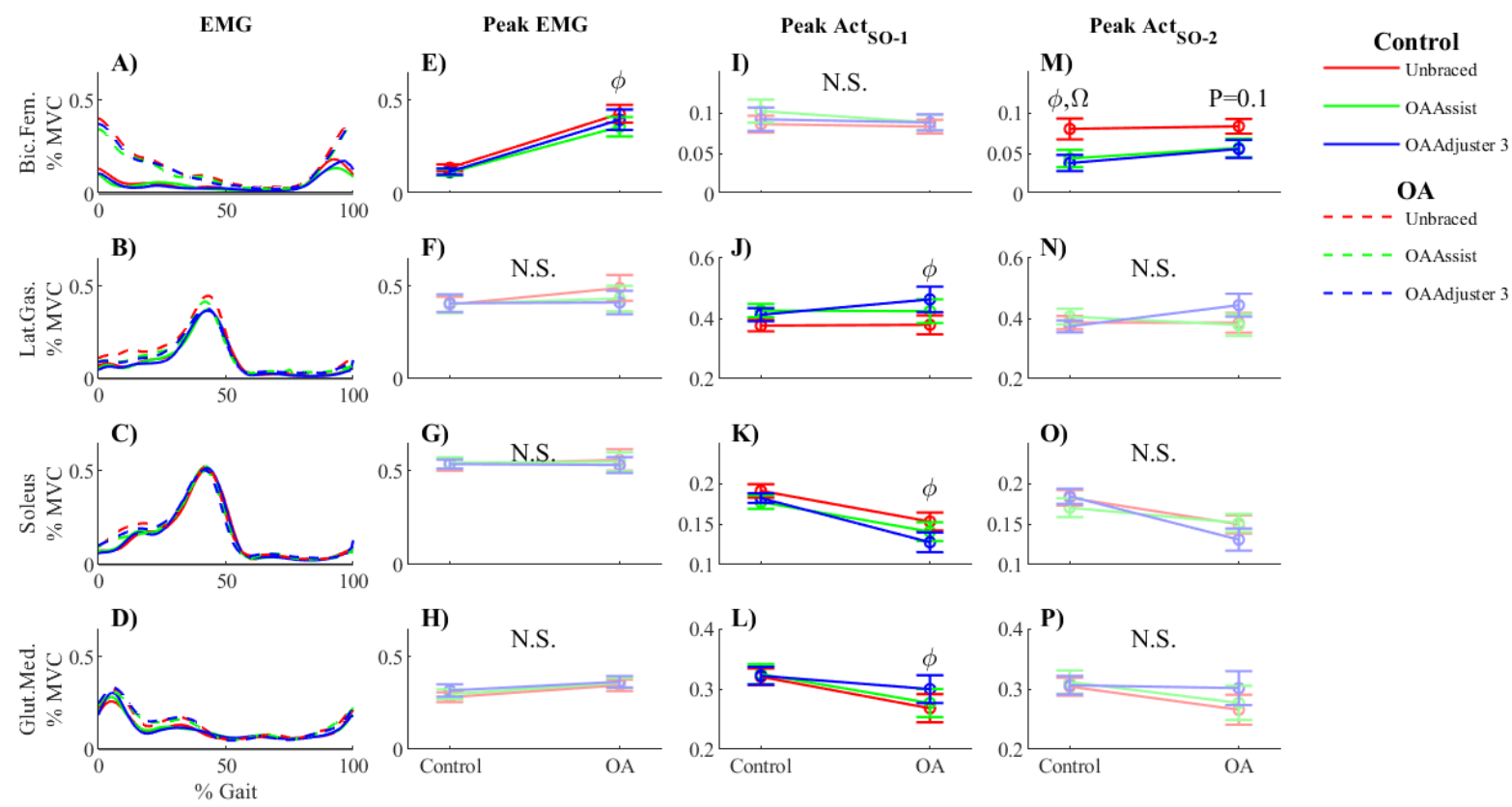
Figure 5A,E). However, this decrease was not predicted by the initial static optimization solution $(\mathrm{SO}-1, \mathrm{P}=0.35$,
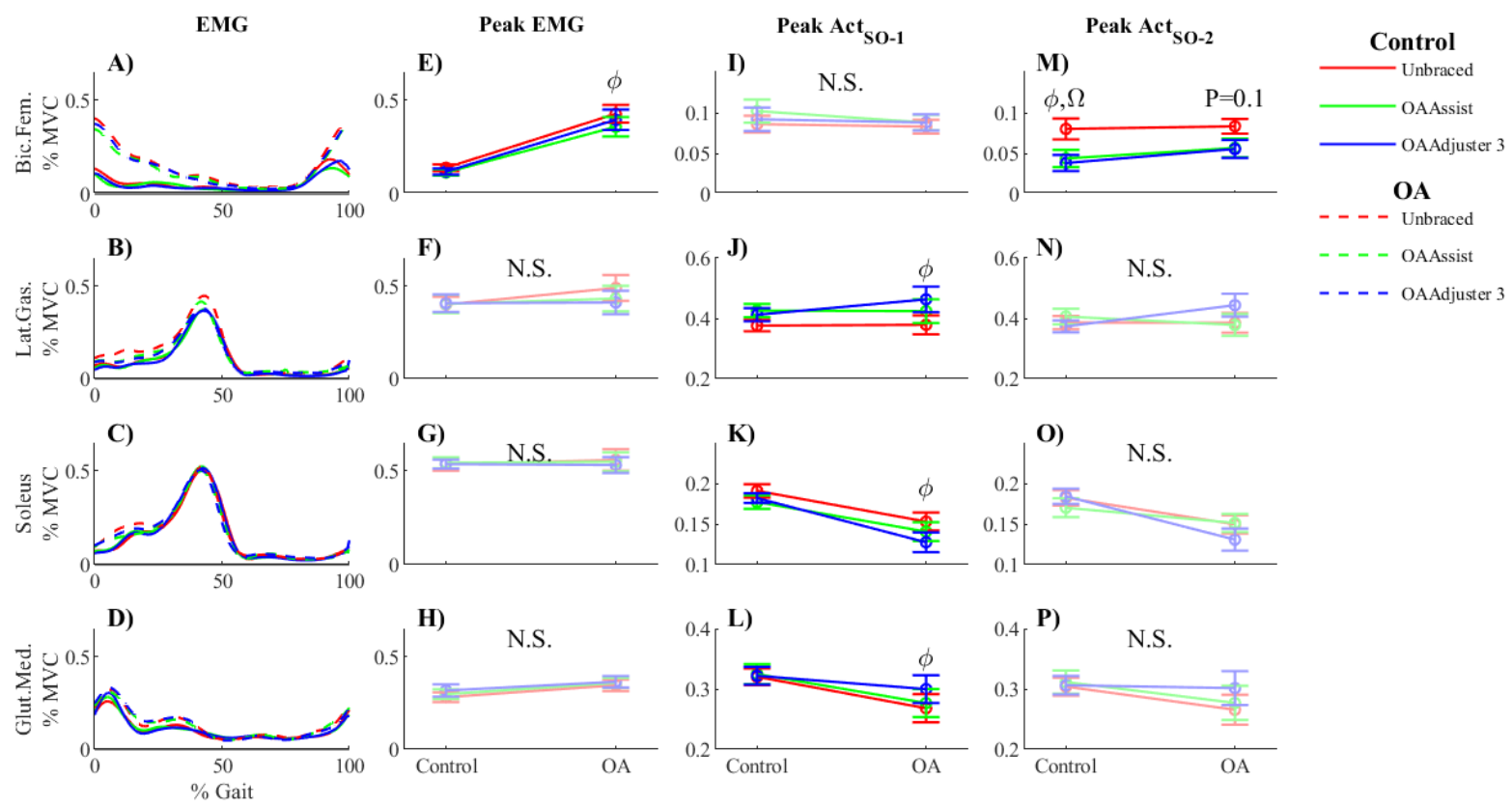

Figure 5I). Furthermore, in contrast with EMG measurements, the initial solution (SO-1) predicted significant differences in muscle activation for lateral gastrocnemius (
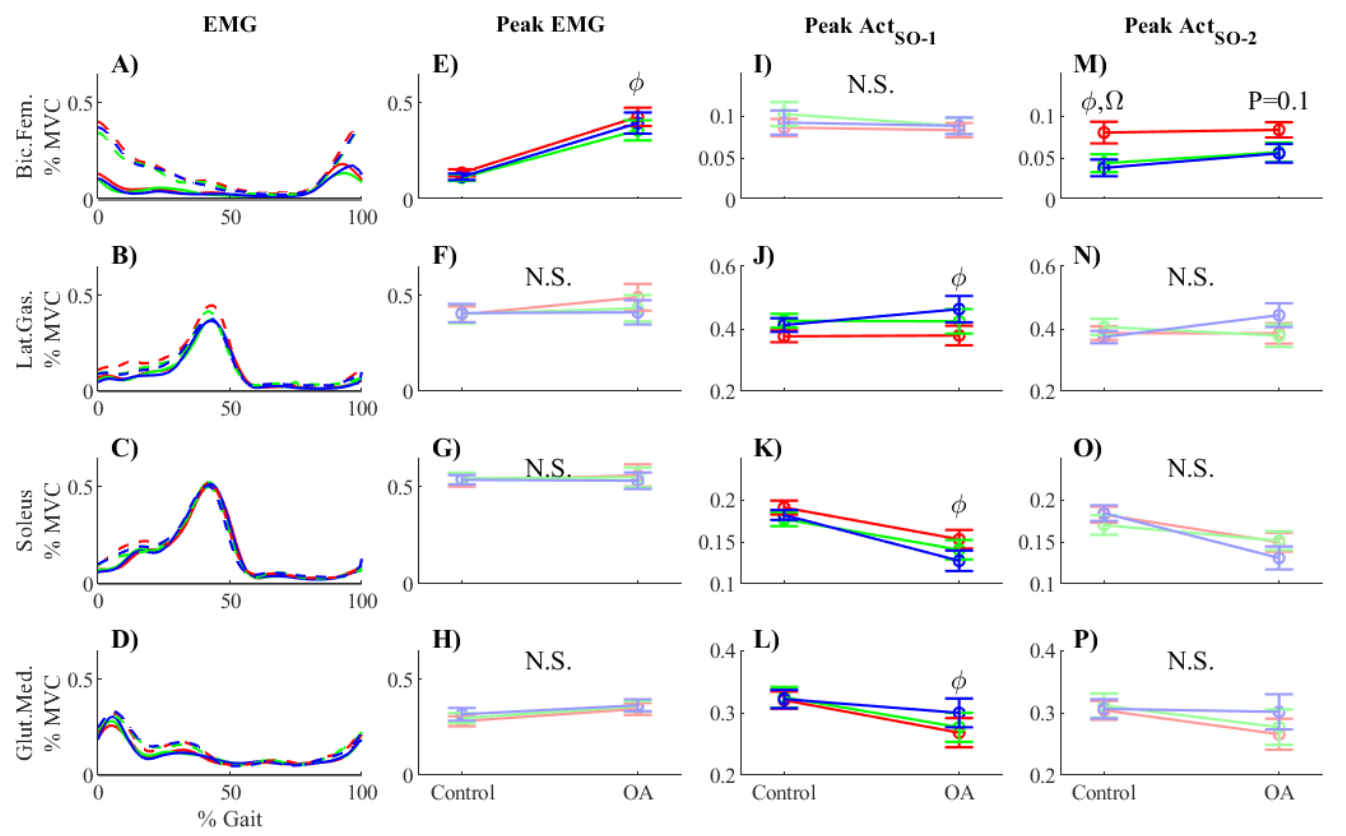

Figure 5J), soleus ( 

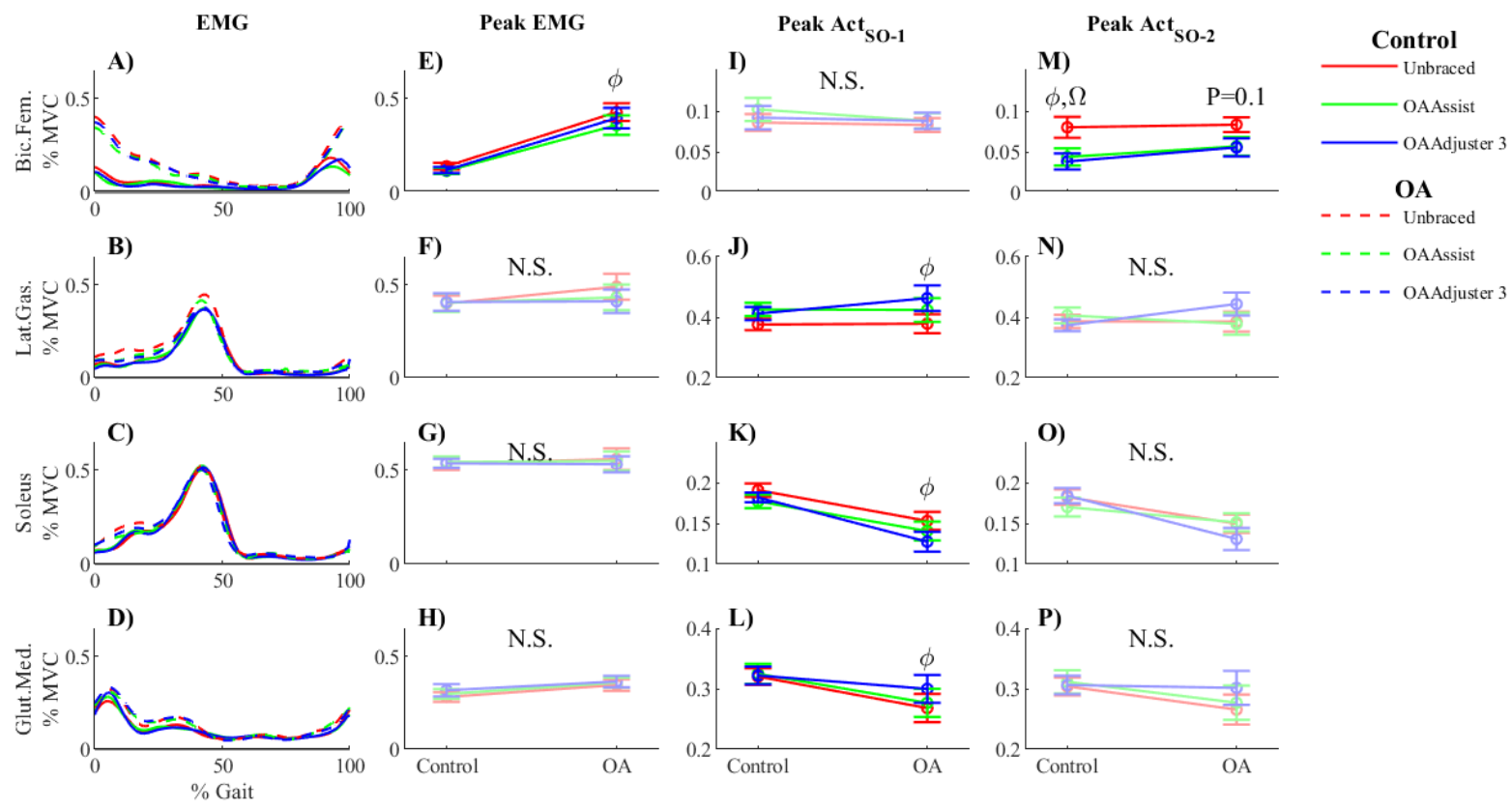

Figure 5K), and gluteus medius (
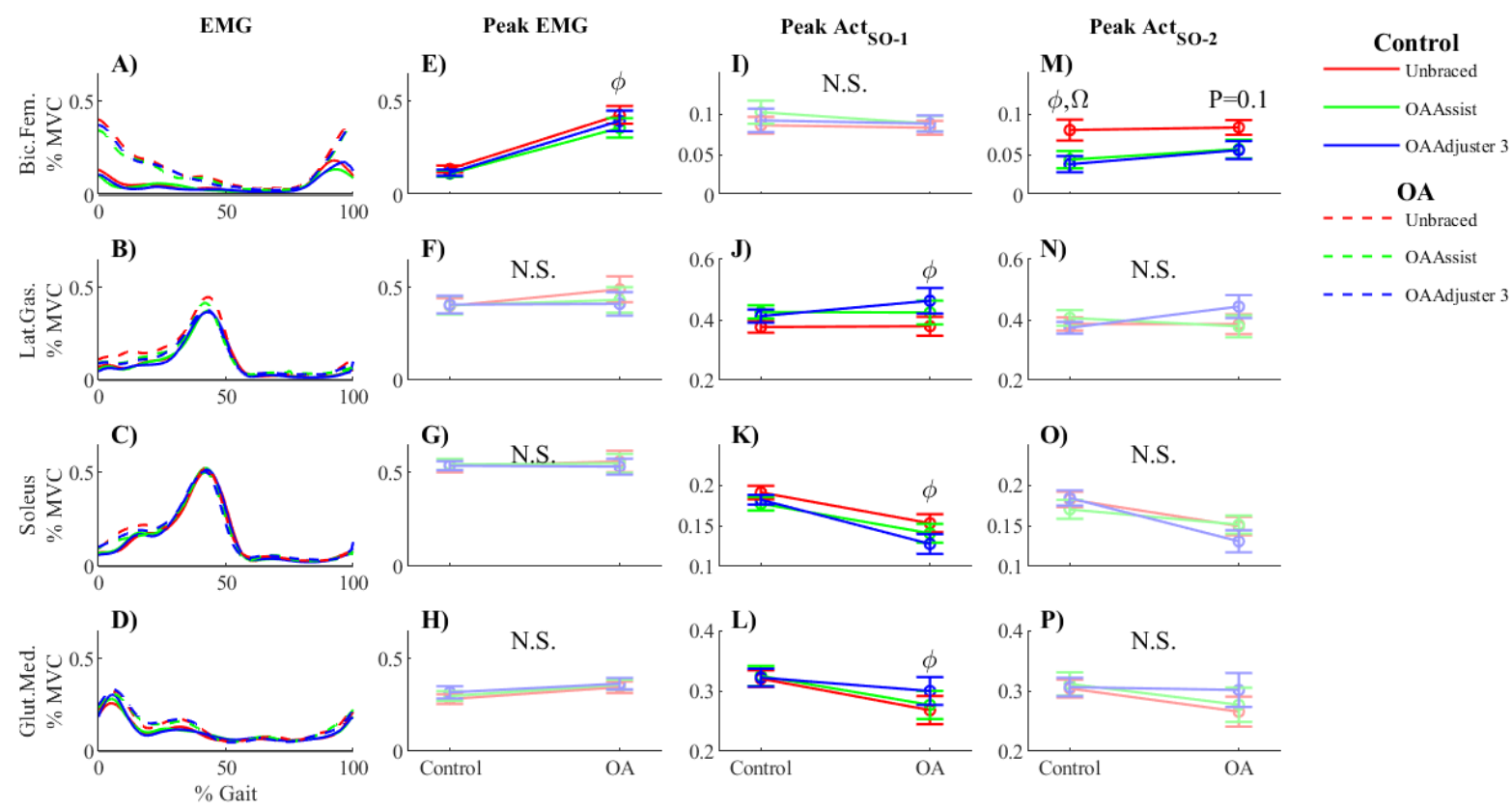

Figure 5L). 


\subsection{Follow-up simulation}

When muscle activations were constrained to resolve these discrepancies with EMG

(Supplementary Material), predicted activations (SO-2,
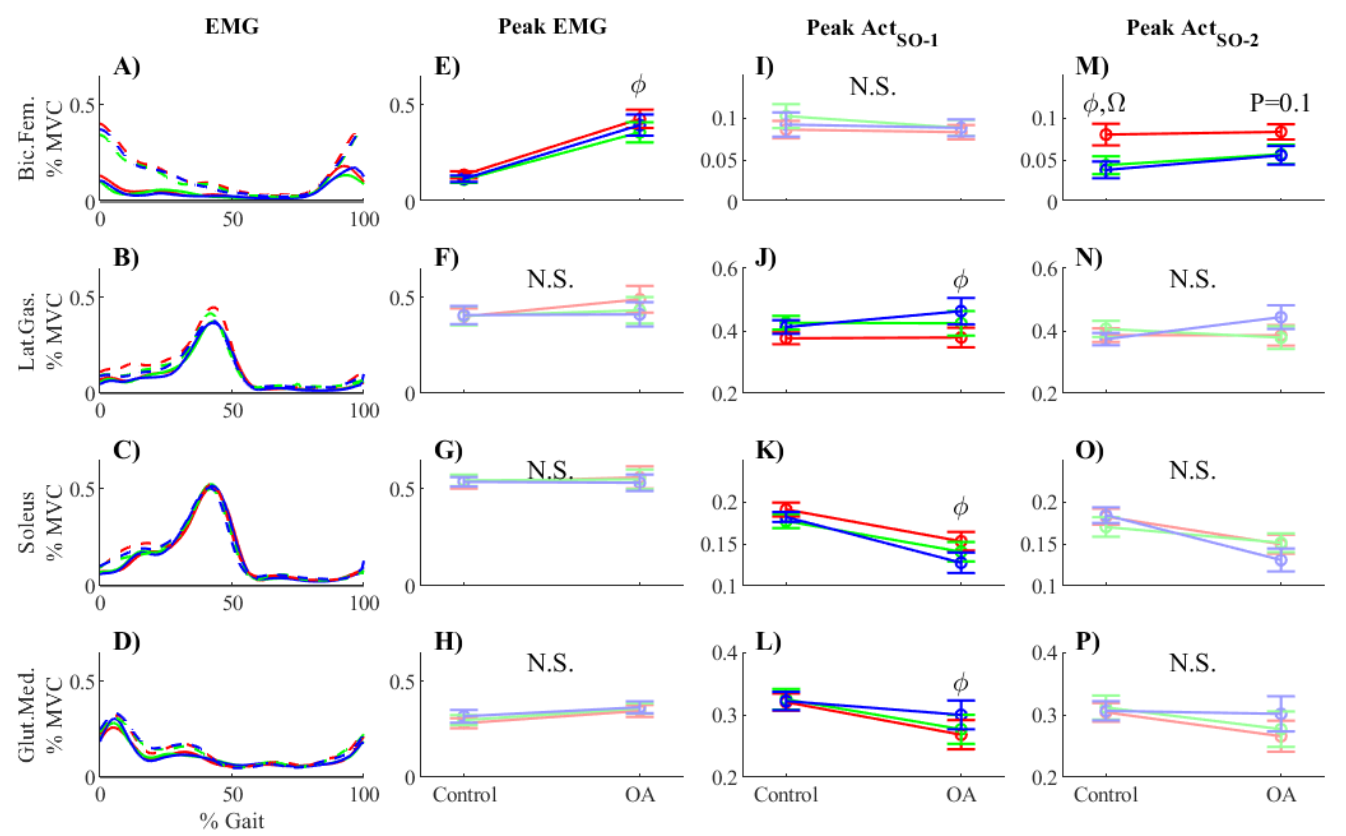

Figure 5M-P) were similar in magnitude to the original solution (SO-1,
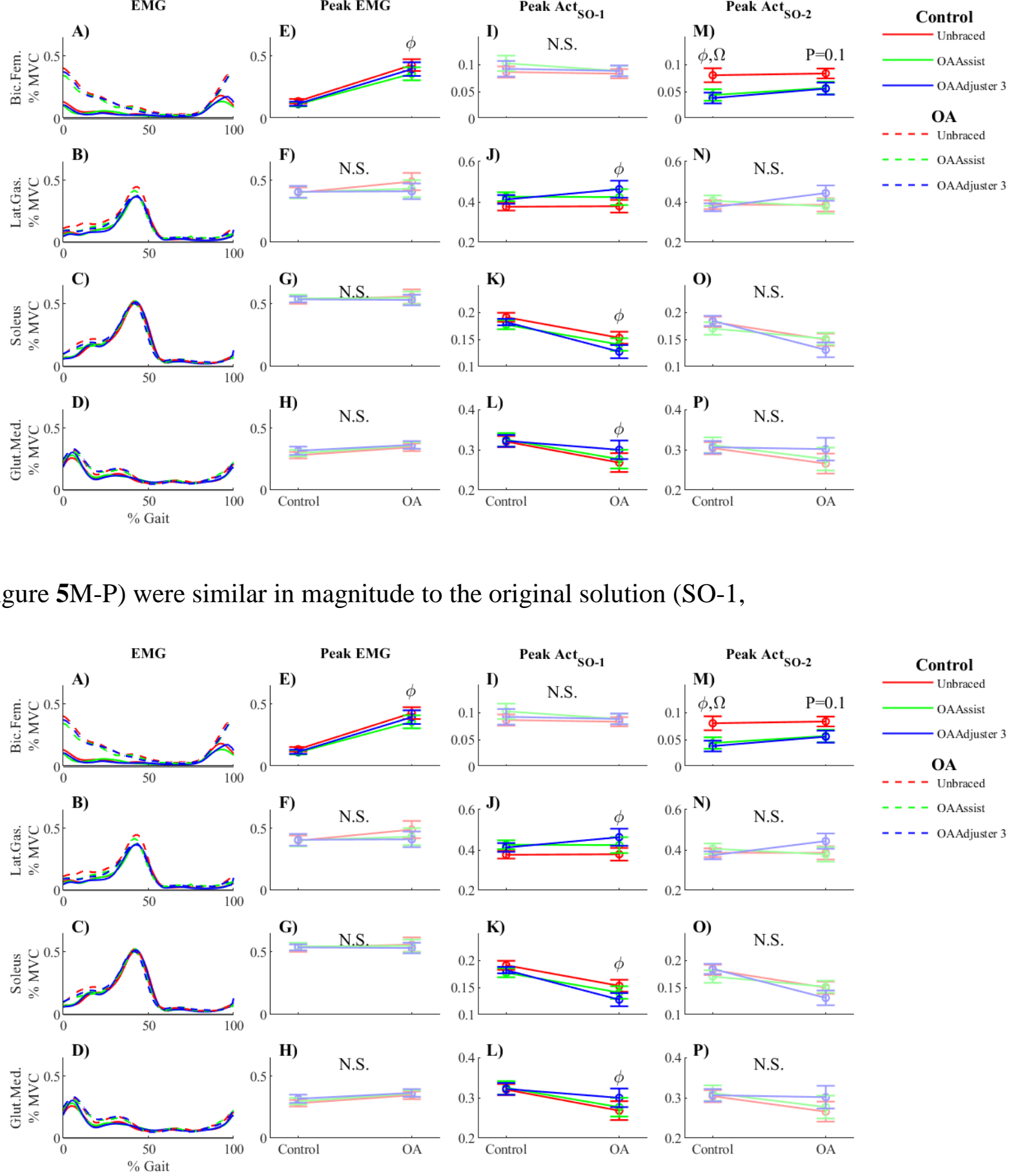

- - OAAdjuster 
Figure 5I-L), and the change in contact force between braced and unbraced conditions was unaltered $(\mathrm{P}>0.76$, 

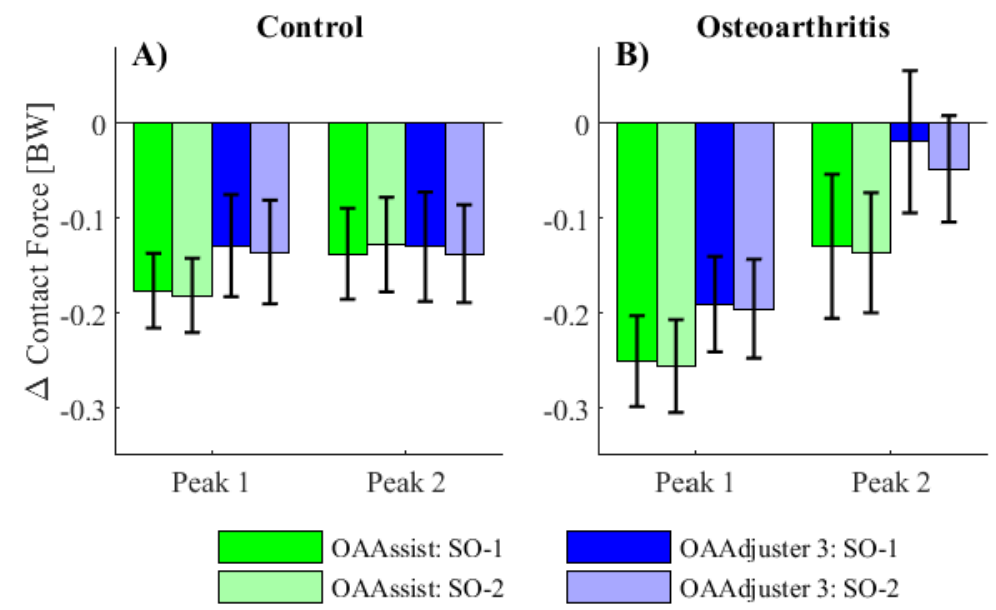

Figure 6). Therefore, contributions to joint contact loads were interpreted only for the initial (SO-1) static optimization.

\subsection{Contributions to Joint Contact Loads}

In the unbraced condition, the primary contributor to medial knee contact loading was the external adduction moment ( 

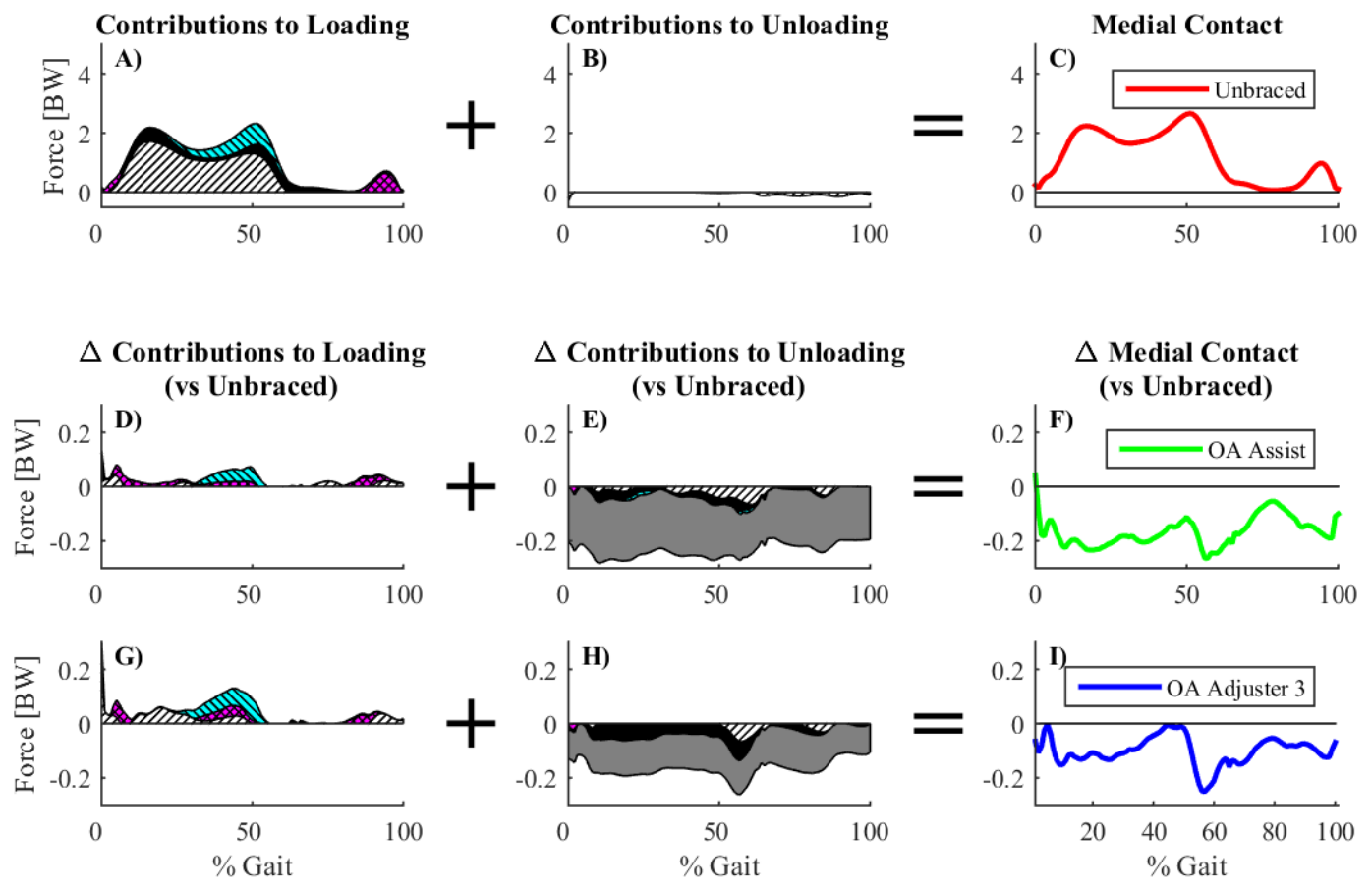

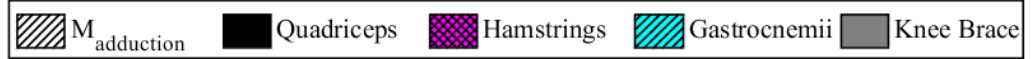

Figure 7A-C). Medial contact forces were increased by quadriceps forces in early-stance and gastrocnemii in late-stance. Hamstrings played a minor role at the start and end of the gait cycle, which was not aligned with early- or late-stance peak loads.

When subjects were wearing either knee brace, the abduction moment applied by the knee brace was the primary contributor to unloading the medial knee joint ( 

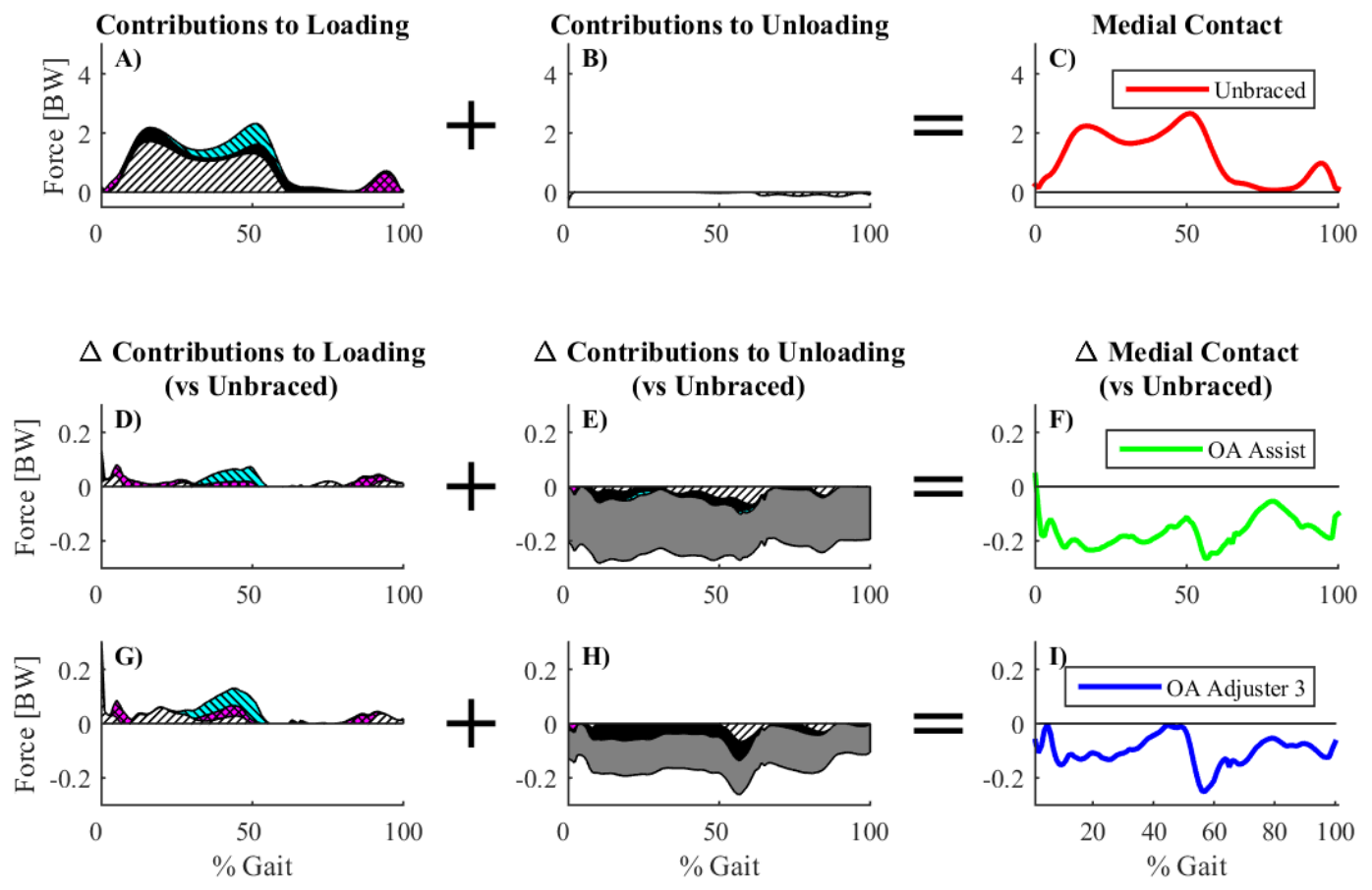

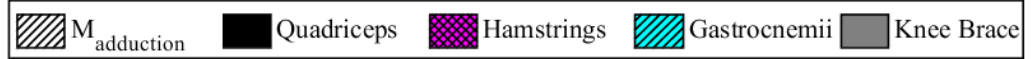

Figure 7E,H). The quadriceps contribution decreased in braced versus unbraced conditions, which indicates slightly decreased quadriceps force ( 

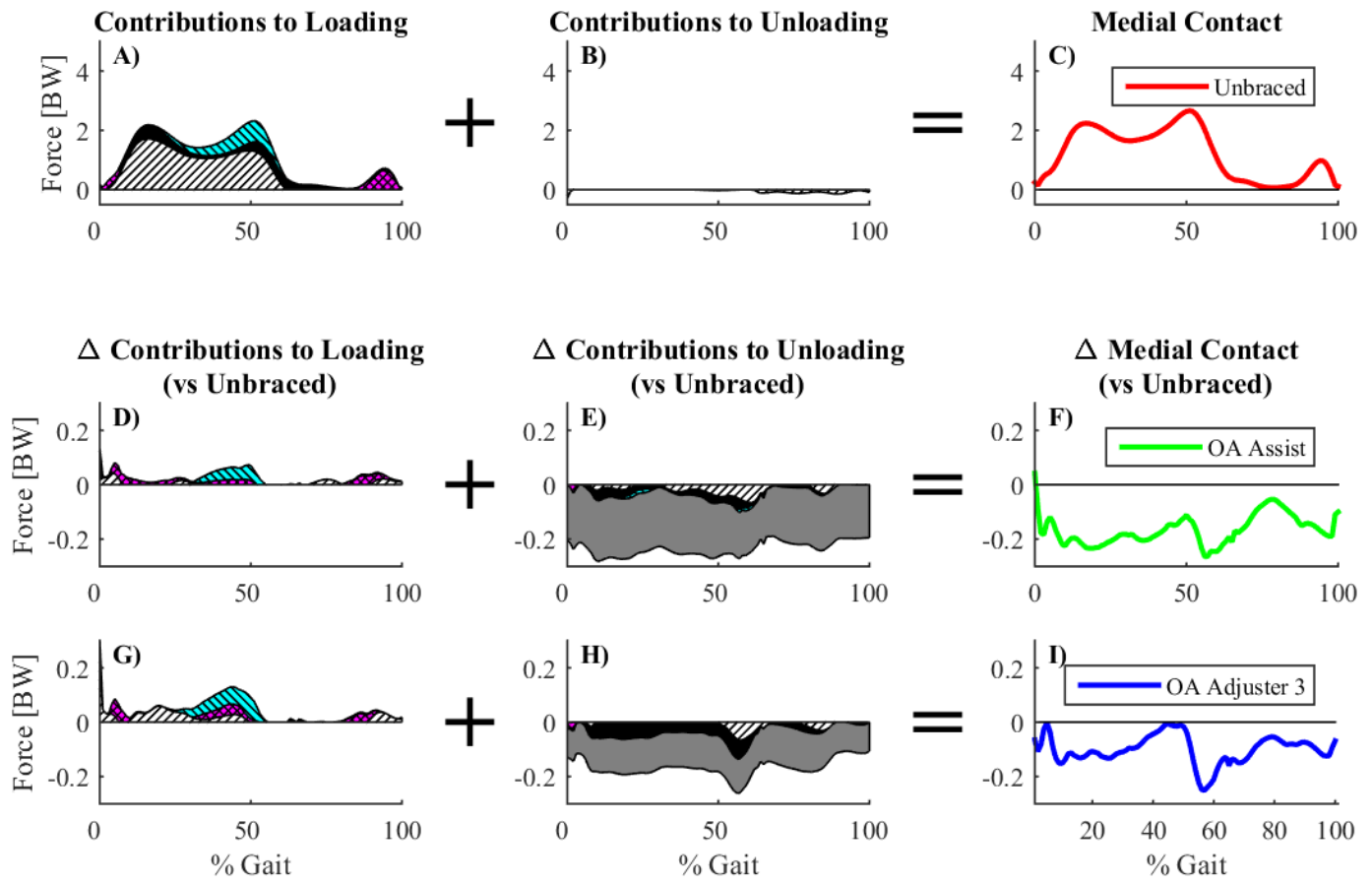

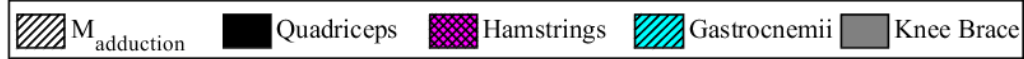

Figure 7E,H) due to statistically insignificant $(\mathrm{P}>0.3)$ decreases in first peak knee flexion moments (
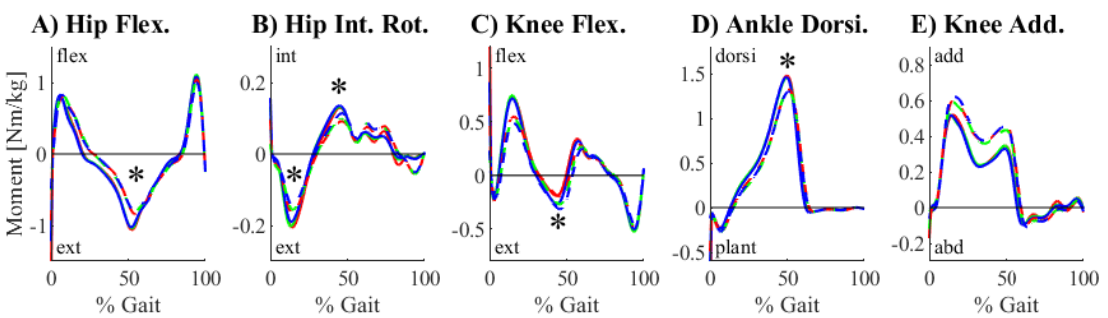

F) Knee + Brace Abd.
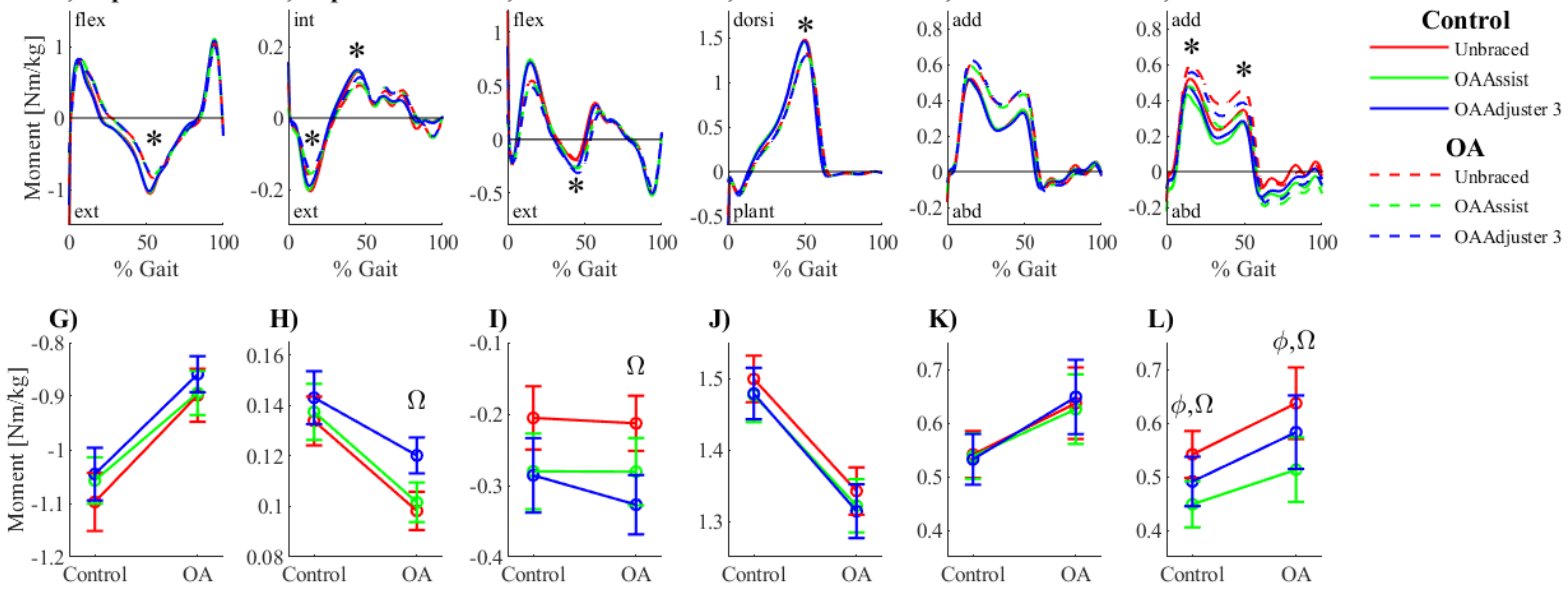

Figure 3C). However gastrocnemii contributions were greater in braced than unbraced conditions ( 

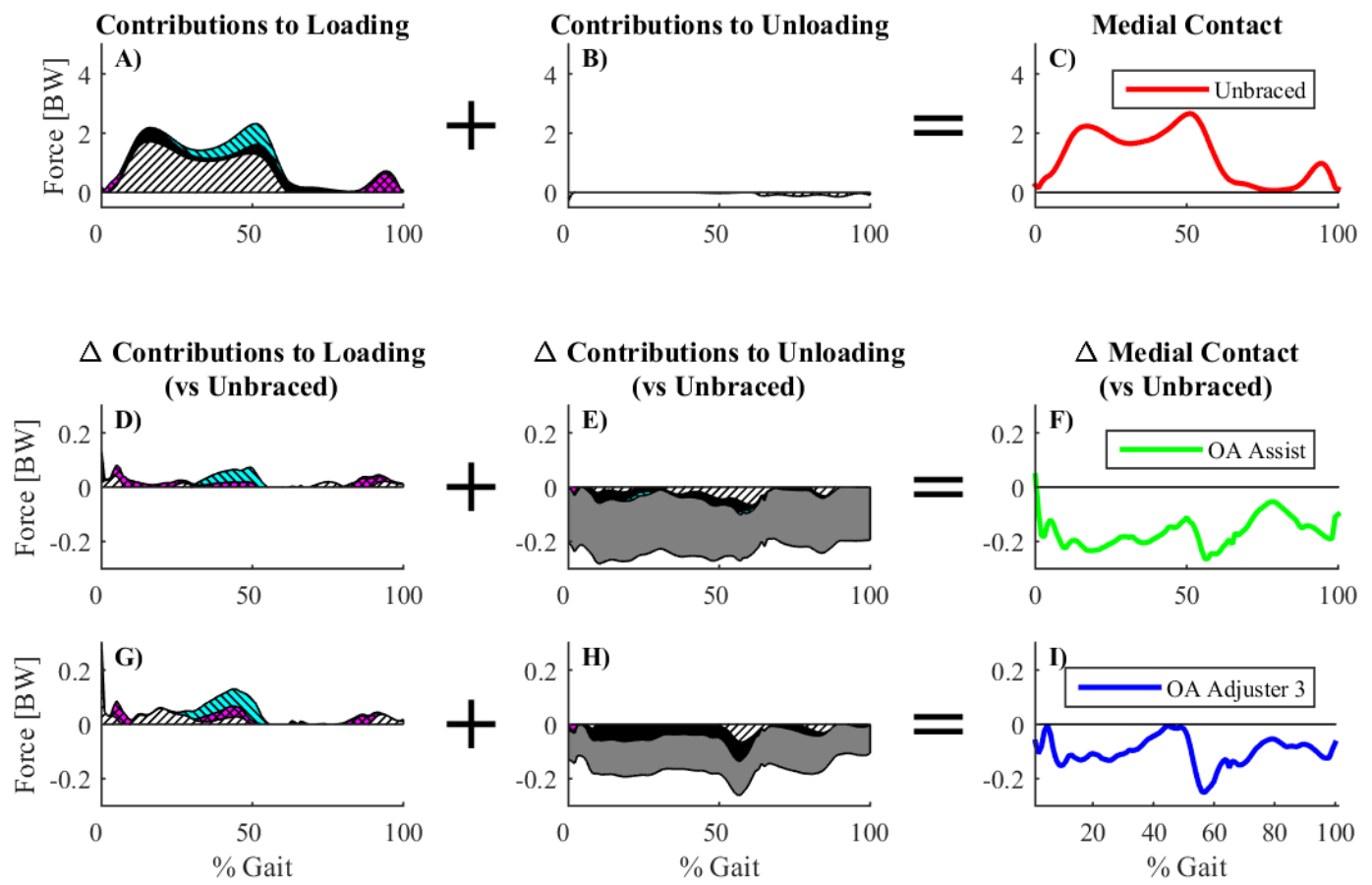

WIIA $\mathrm{M}_{\text {adduction }} \quad$ Quadriceps Hamstrings $\mathbb{Z}$ Gastrocnemii $\square$ Knee Brace

Figure 7D,G), which inhibited the unloading effect of the braces. The OA Assist brace achieved a greater reduction in medial contact force than the OA Adjuster 3 brace ( 

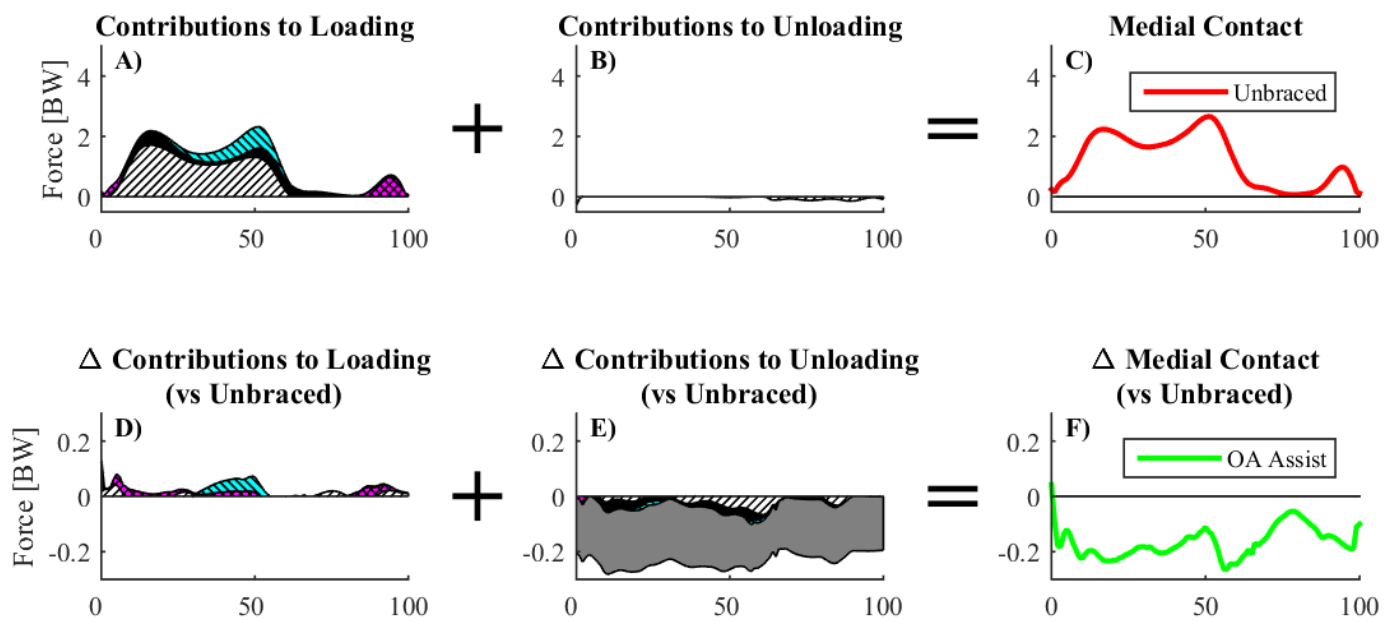

$\Delta$ Contributions to Unloading
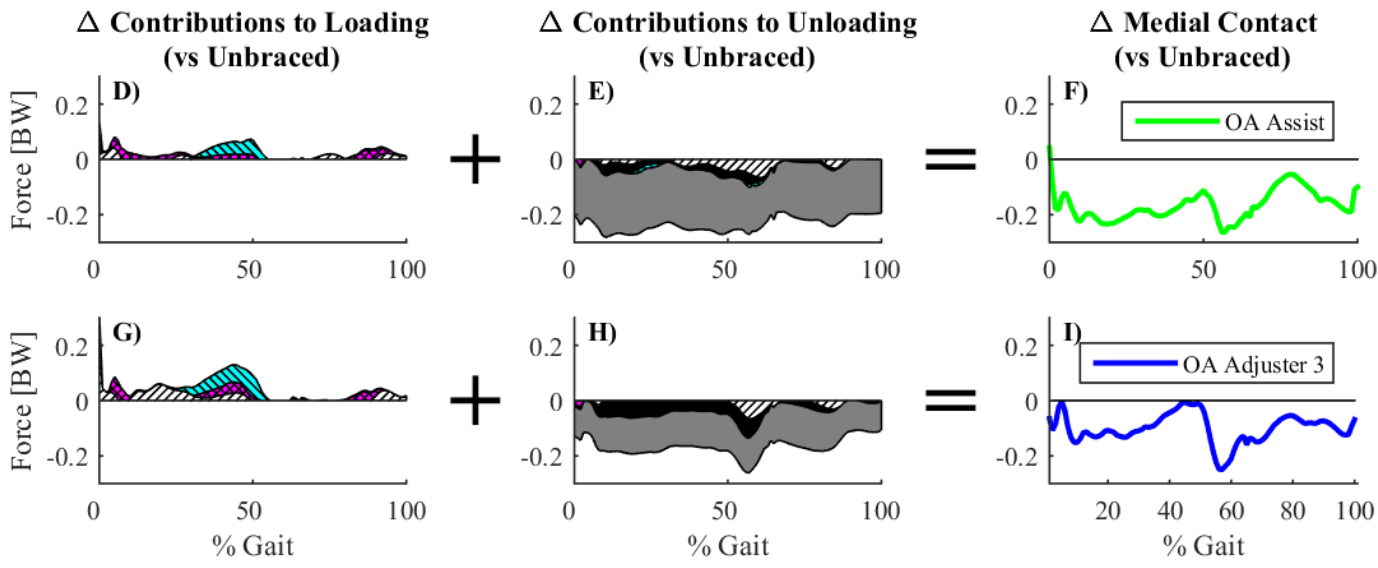

WIIA $\mathrm{M}_{\text {adduction }} \quad$ Quadriceps Hamstrings $\mathbb{Z}$ Gastrocnemii $\square$ Knee Brace

Figure 7F vs I) primarily by applying a greater abduction moment ( 

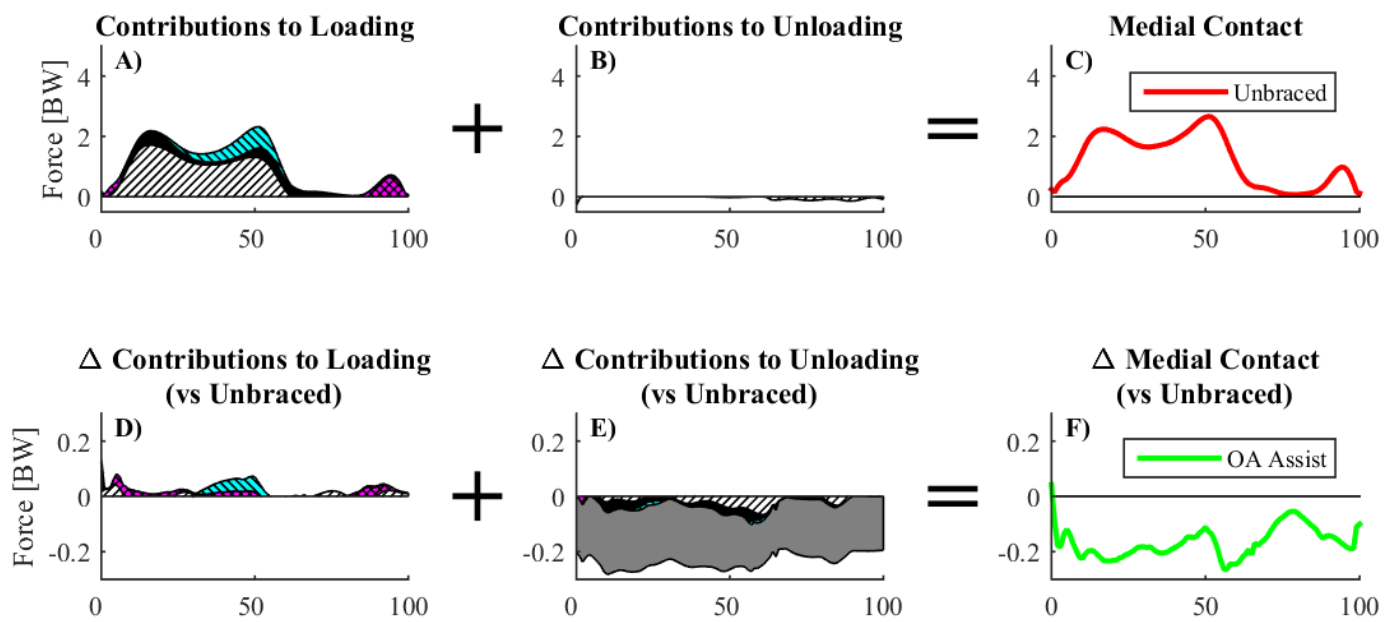

$\Delta$ Contributions to Unloading

$\Delta$ Medial Contact (vs Unbraced)

(vs Unbraced)
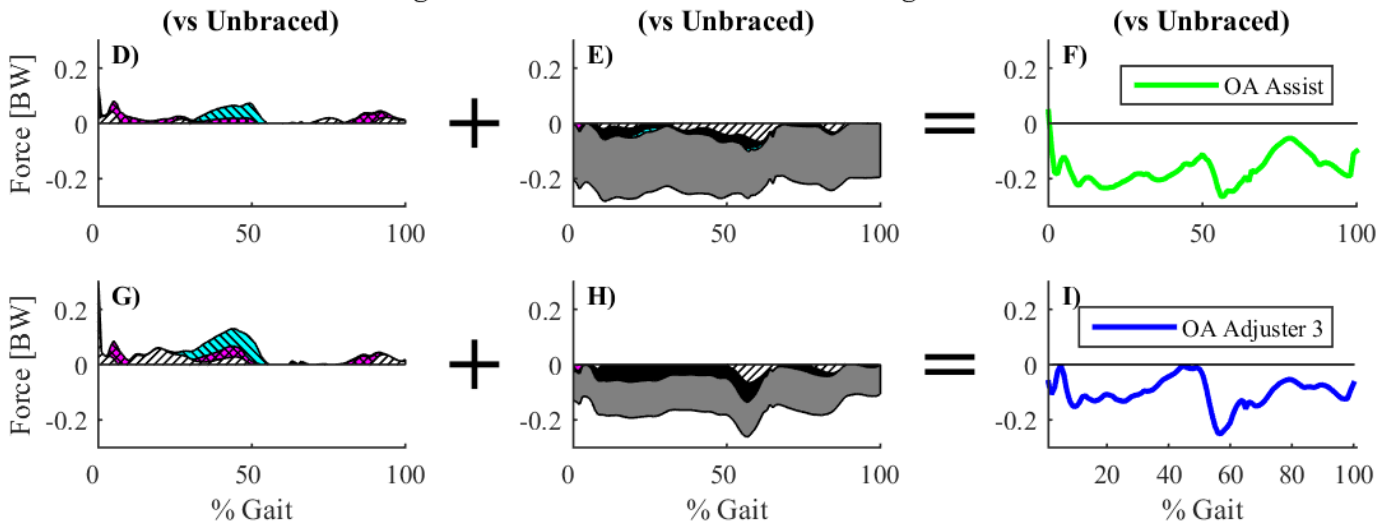

WIIA $\mathrm{M}_{\text {adduction }} \quad$ Quadriceps Hamstrings $\mathbb{Z}$ Gastrocnemii $\square$ Knee Brace 
A) OA Assist

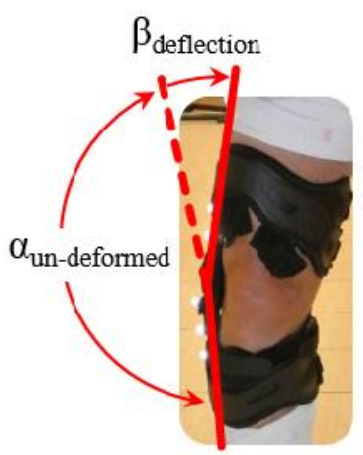

B) OA Adjuster 3
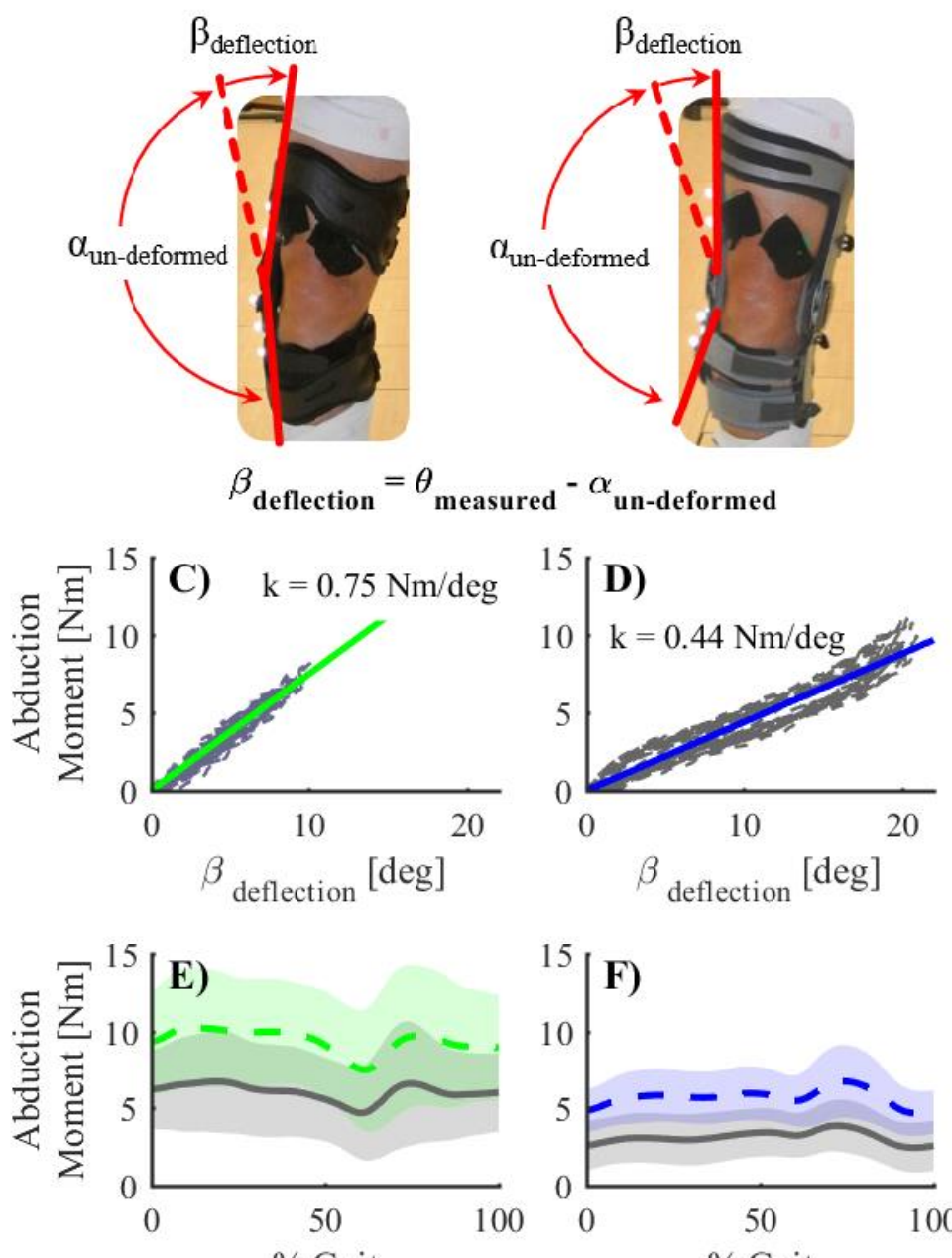

$\%$ Gait

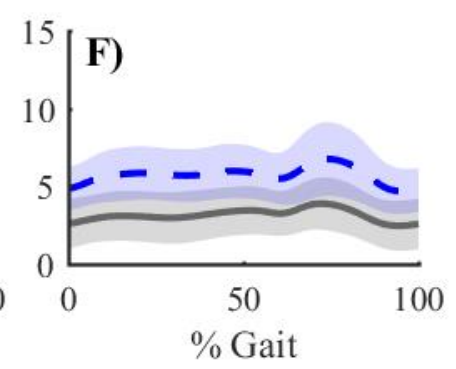

Figure 1E vs F).

\section{Discussion}

Knee bracing decreased peak medial knee contact loads by an estimated 0.1-0.3 BW (Table 3) in osteoarthritis and control subjects during overground gait. This medial load reduction was primarily caused by the unloading abduction moment applied by the brace to the knee joint ( 

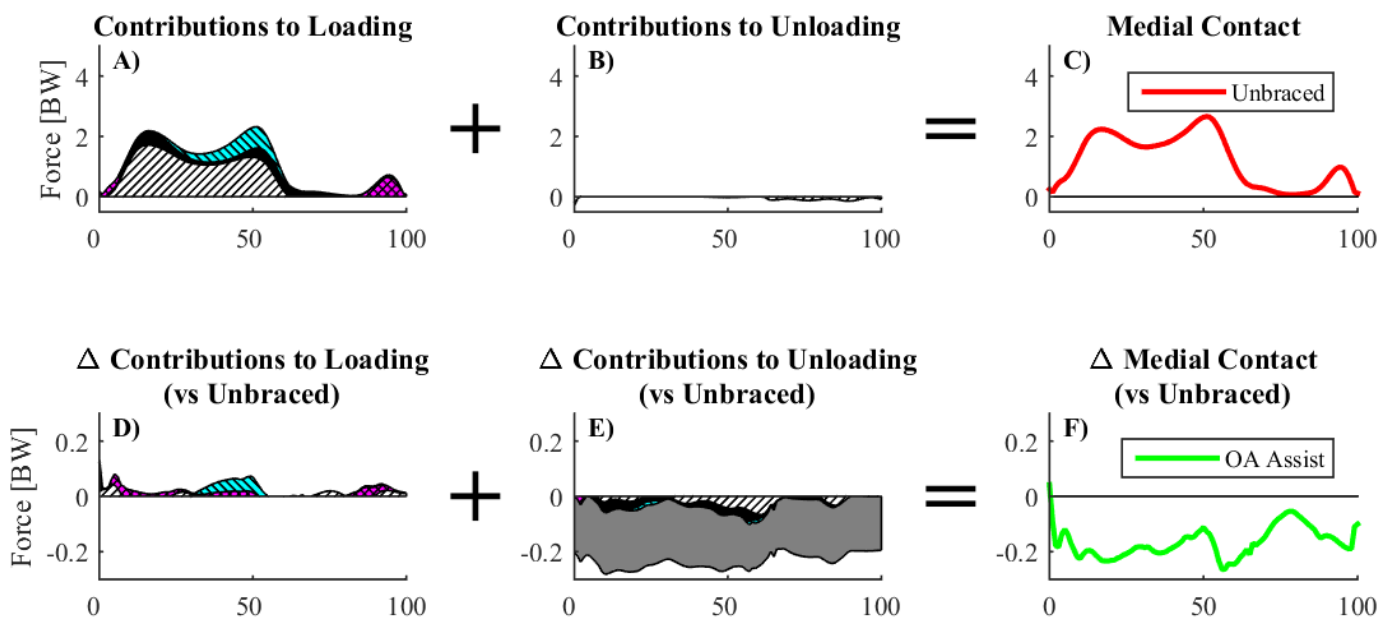

$\Delta$ Contributions to Unloading (vs Unbraced)
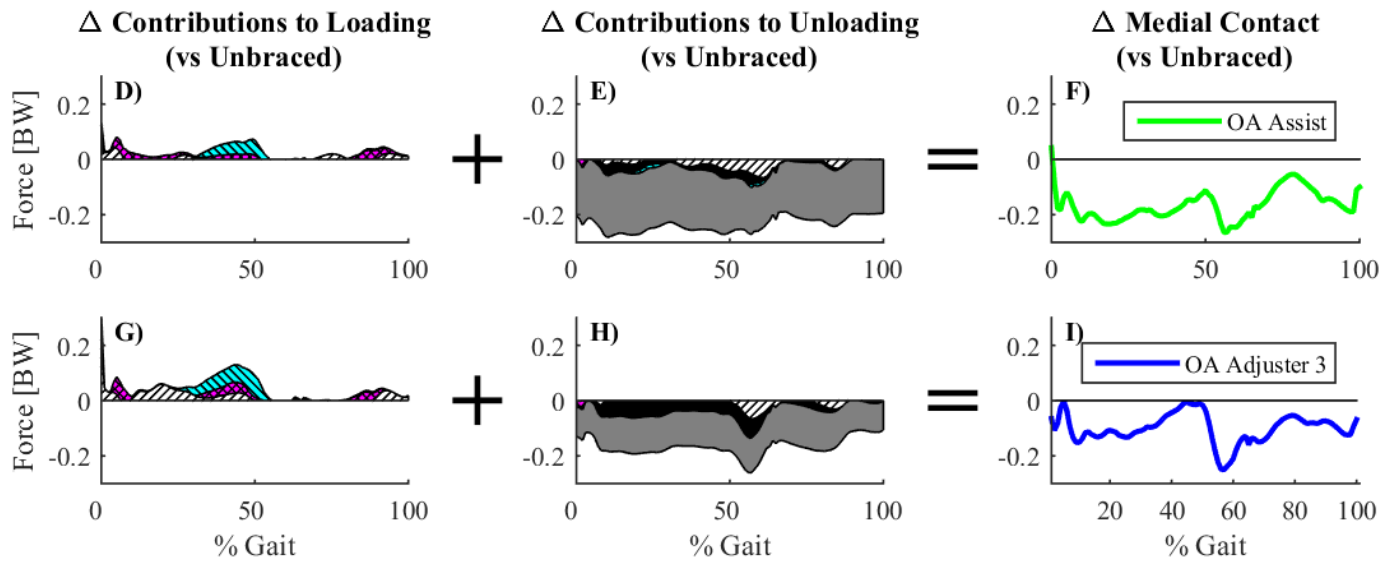

WIIA $\mathrm{M}_{\text {adduction }} \quad$ Quadriceps Hamstrings

Figure 7). The model revealed no change in the total knee contact load; therefore, the primary effect of bracing was to shift loading from the medial to the lateral condyle (
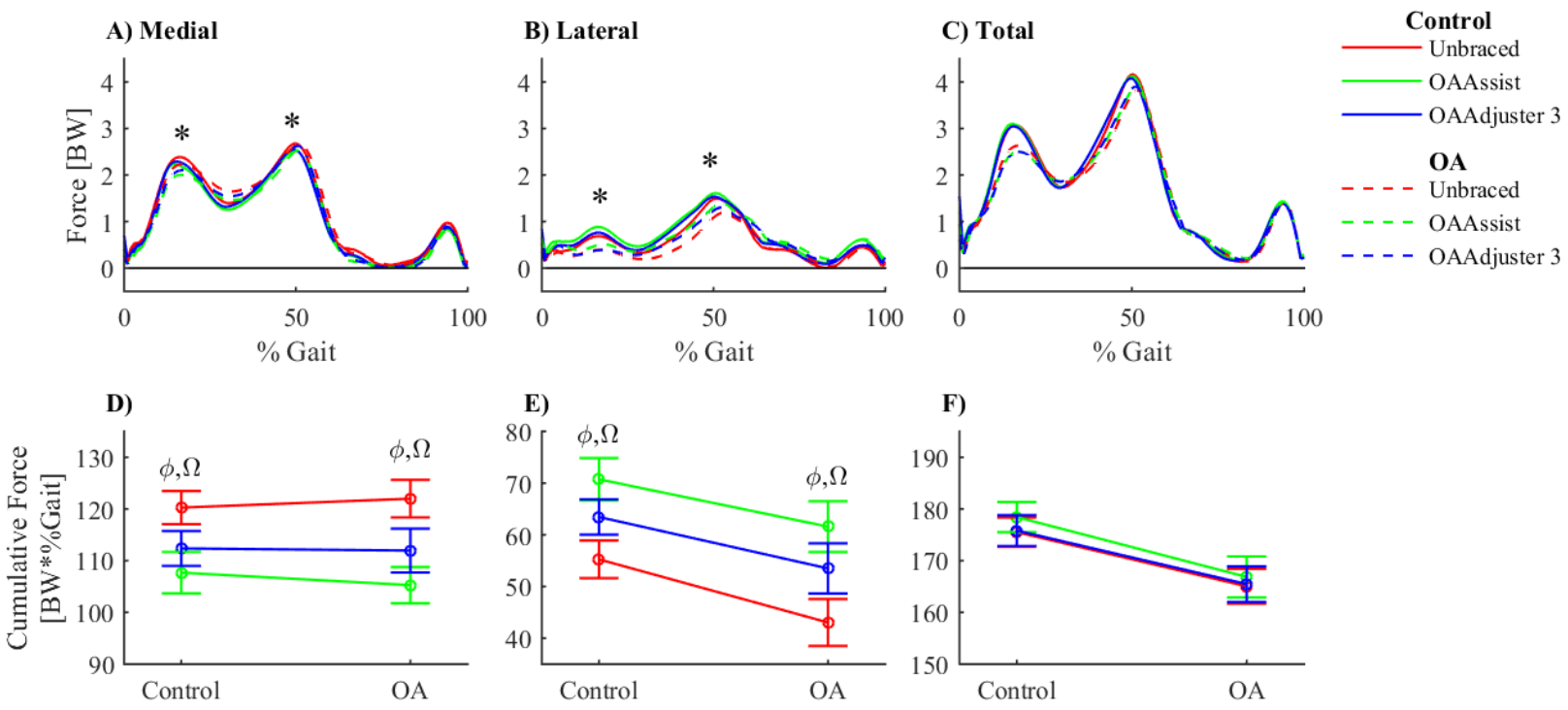

Figure 4). 
Unbraced peak contact loads predicted in this study $($ medial $=2.7 \mathrm{BW}$, total $=4.6 \mathrm{BW}$,
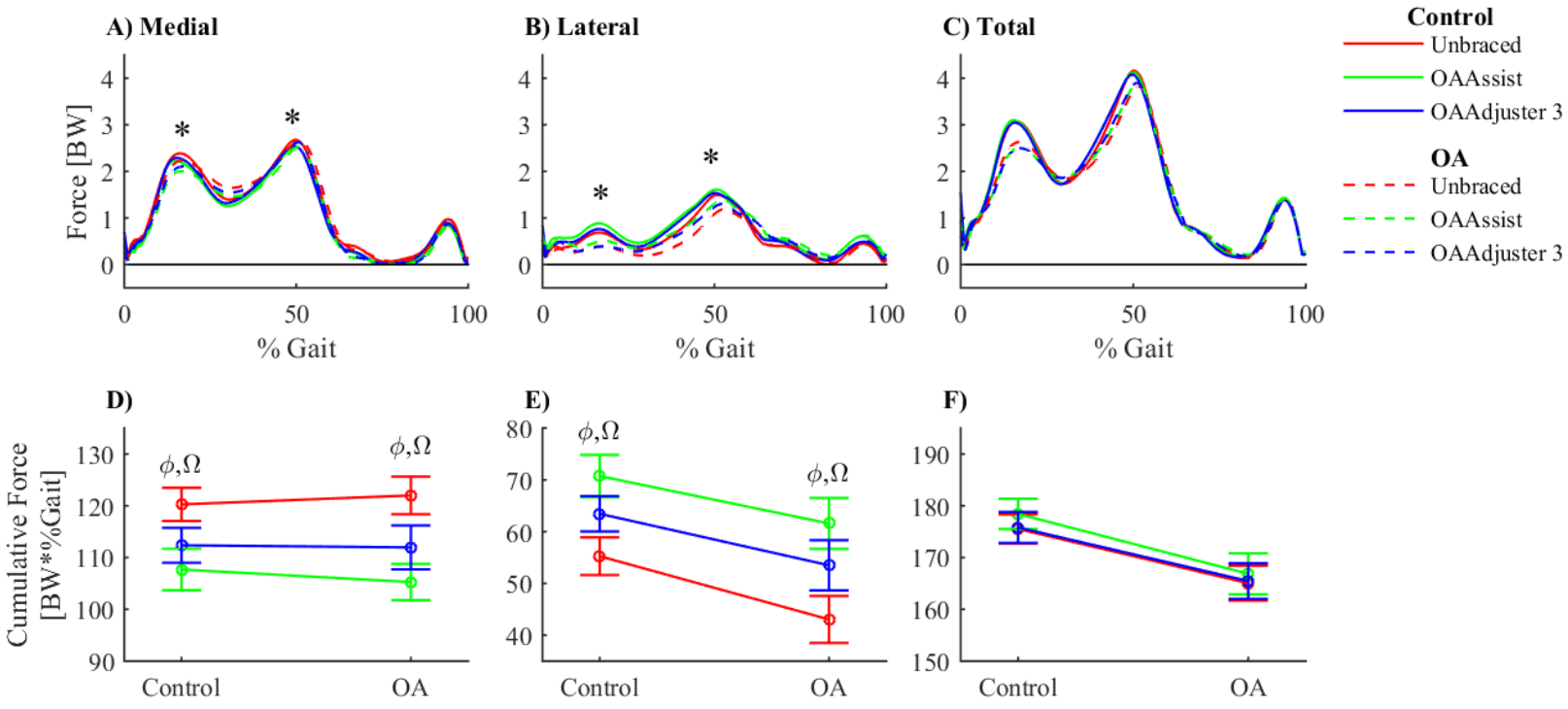

Figure 4) were similar to previous model estimates (total $=4.4 \mathrm{BW}$, Richards and Higginson 2010), but greater than in vivo medial and total loads of $1.95 \mathrm{BW}$ and $2.6 \mathrm{BW}[50,51]$. Walking speeds in the current study $(\sim 1.4 \mathrm{~m} / \mathrm{s})$ were faster than those reported for the in vivo study $(1.1 \mathrm{~m} / \mathrm{s}$, Kutzner et al. 2013) which would slightly increase joint loads [52]. However, the majority of this error can likely be attributed to the use of a simplified musculoskeletal model [53], and in particular excessive recruitment of bi-articular gastrocnemius versus uni-articular soleus muscles [54]. Interestingly, osteoarthritis subjects walked approximately $0.2 \mathrm{~m} / \mathrm{s}$ slower than healthy controls (Table 1). While slower walking has been proposed as a mechanism to alleviate both knee contact forces and pain [49], osteoarthritis subjects in the present study did not significantly reduce their peak medial knee contact loads ( 

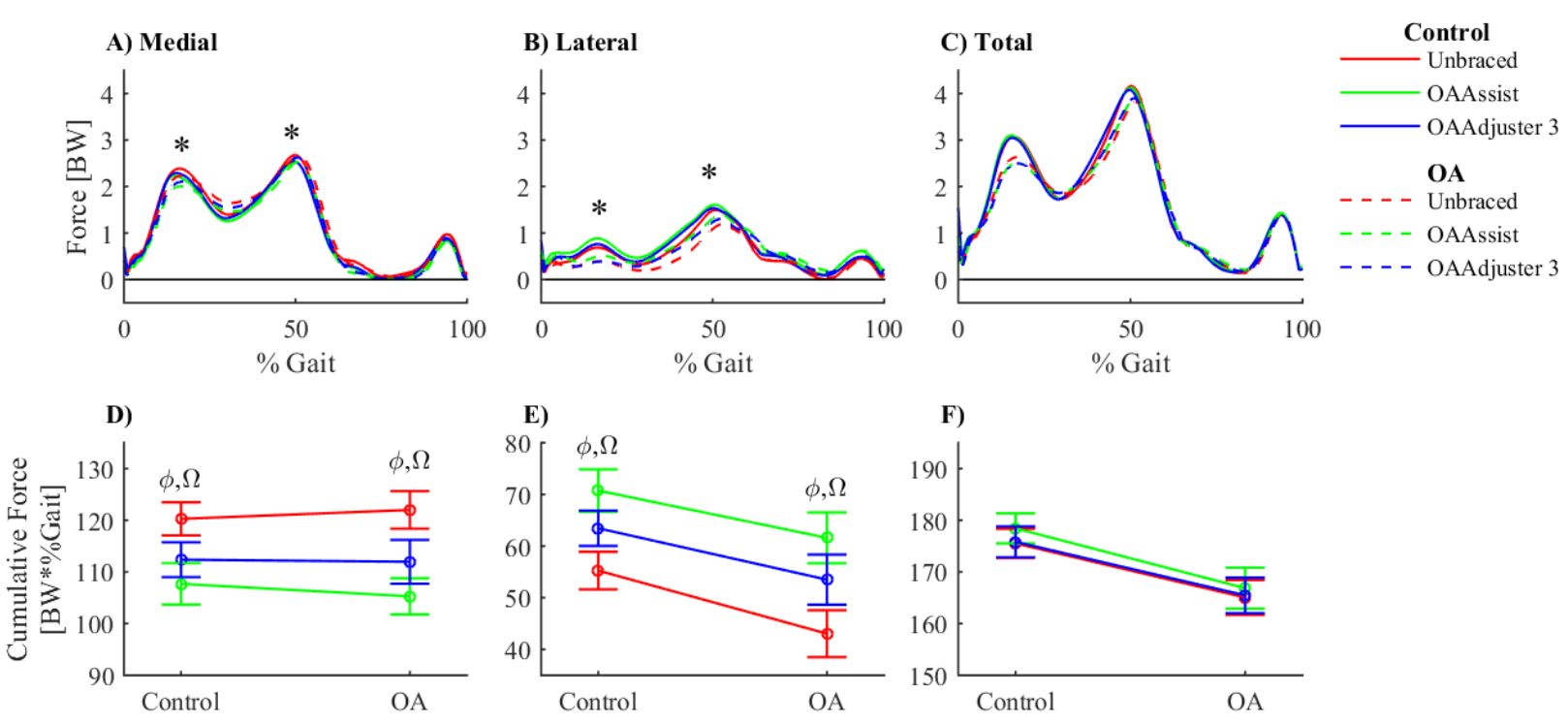

Figure 4). This observation further motivates clinical need for bracing as a load-relief device.

Despite potential errors in predictions of contact force magnitude, our previous validation against in vivo data [40] suggested that even when absolute contact force predictions were inaccurate, relative changes in contact between gait conditions may be more accurate (i.e. within $0.03 \mathrm{BW}$ ). For the braced conditions, we predicted mean reductions in medial loading of roughly 5 to $15 \%$ (Table 3), which is similar to the range of 8 to $17 \%$ reported using a different brace design and a simplified musculoskeletal model [12]. By comparison, in vivo measurements in three subjects revealed mean reductions in medial knee loads of 7 to $23 \%$ during gait, with a range of 0 to $40 \%$, for two different brace designs [10]. In this study, we found a similar range of 0 to 35\%; thus, our model provided realistic predictions of medial load reduction due to knee bracing.

This study was motivated by the hypothesis that medial knee loads are not simply reduced by the applied brace moment, but are also influenced by concomitant changes in gait dynamics and neuromuscular control. However, our detailed musculoskeletal model revealed that the largest contributor to medial load reduction was the abduction moment applied by the brace to the knee ( 

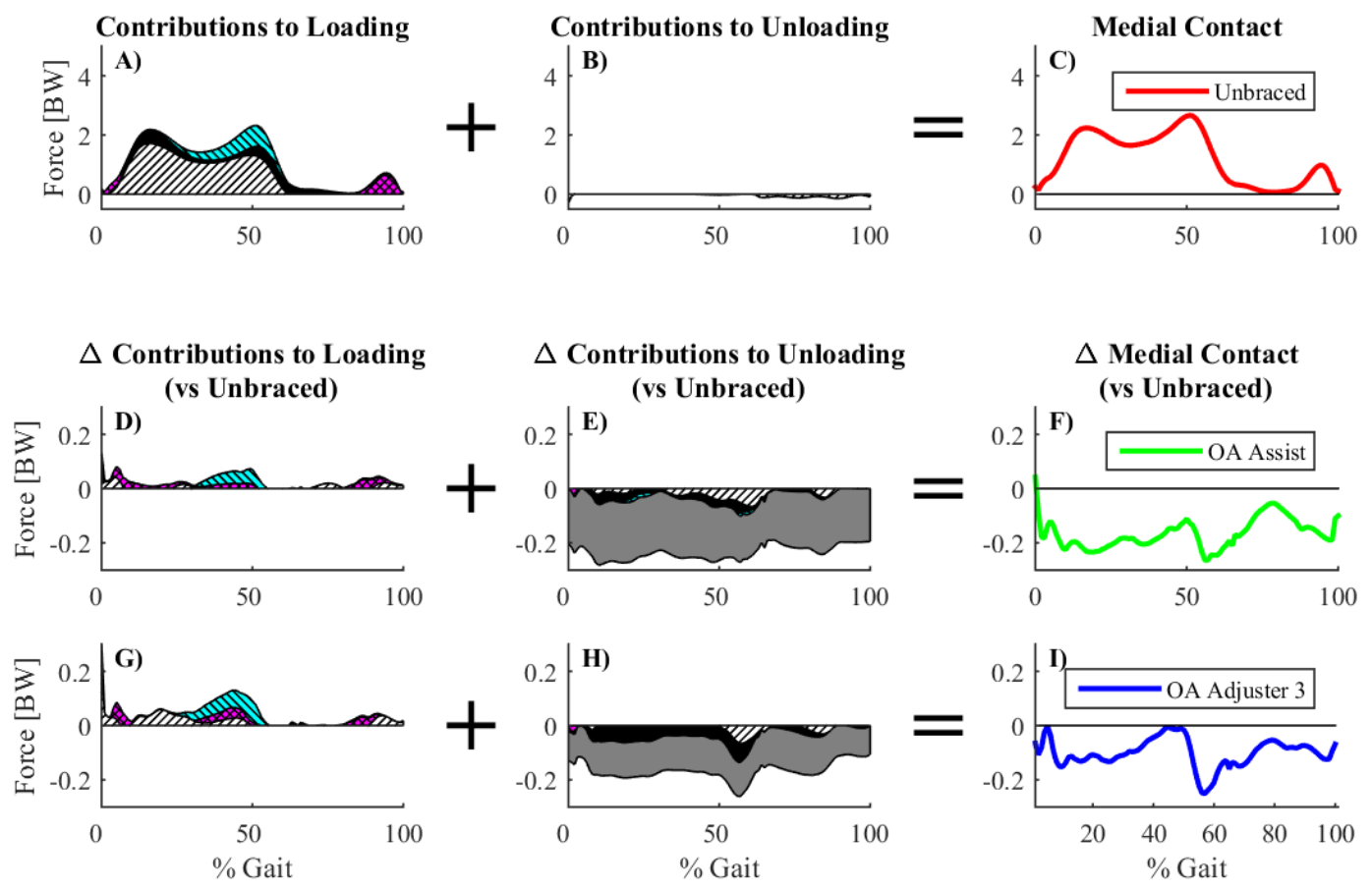

WIIA $\mathrm{M}_{\text {adduction }}$ Quadriceps Hamstrings

Figure 7E,H). Previous studies have reported a decrease in antagonist co-contraction of hamstrings, gastrocnemii, and quadriceps muscles when subjects use an unloader knee brace $[17,20]$. As these muscles span the knee joint, changes in their activation will directly alter compressive joint loads $[55,56]$. In this study, we detected a small decrease only in biceps femoris EMG, which was significant (post-hoc) only for the osteoarthritis subjects. However, due to their timing of activation at the start and end of the gait cycle ( 

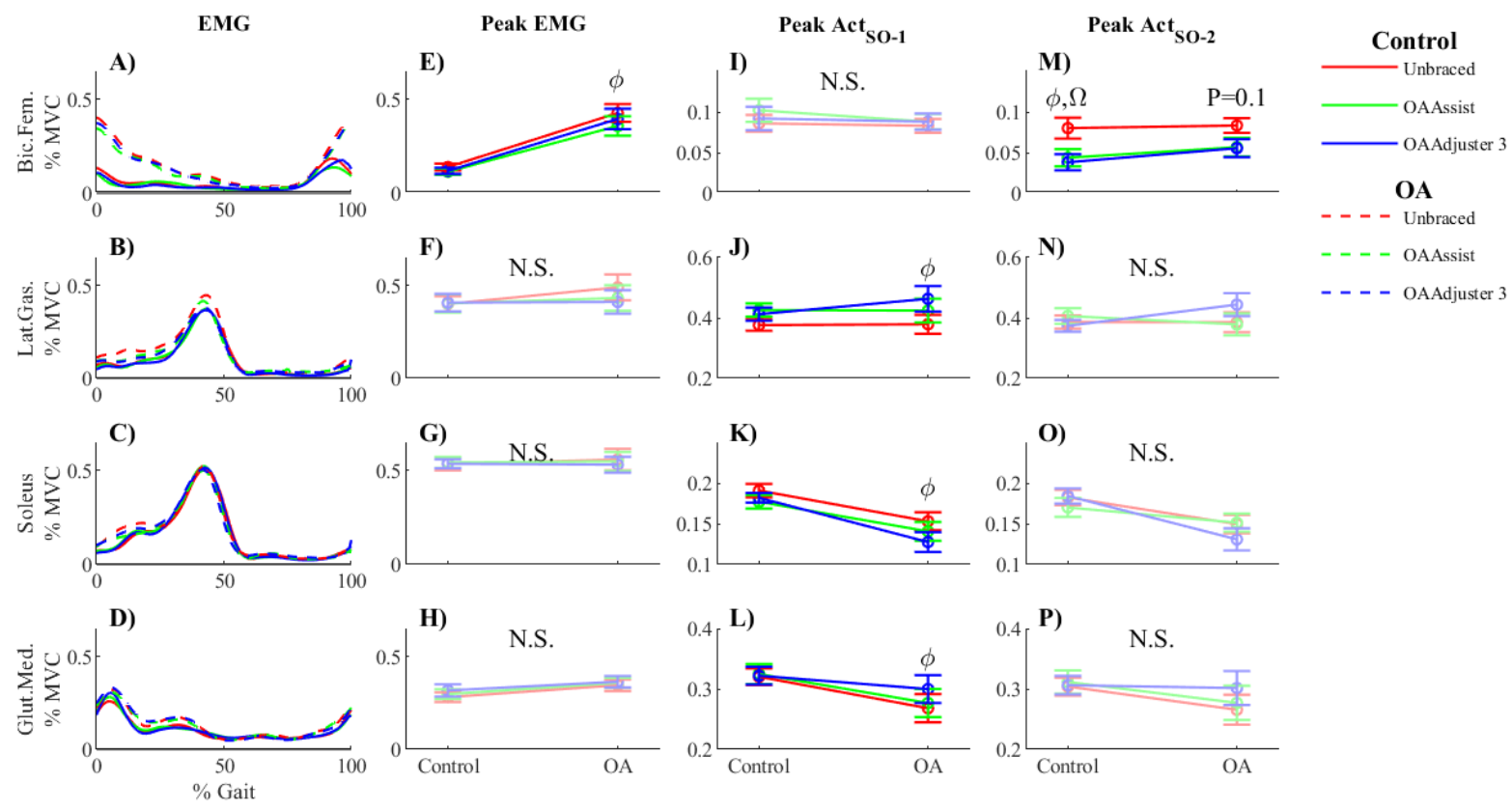

Figure 5) hamstrings muscles did not affect the braces' ability to reduce peak medial loads (
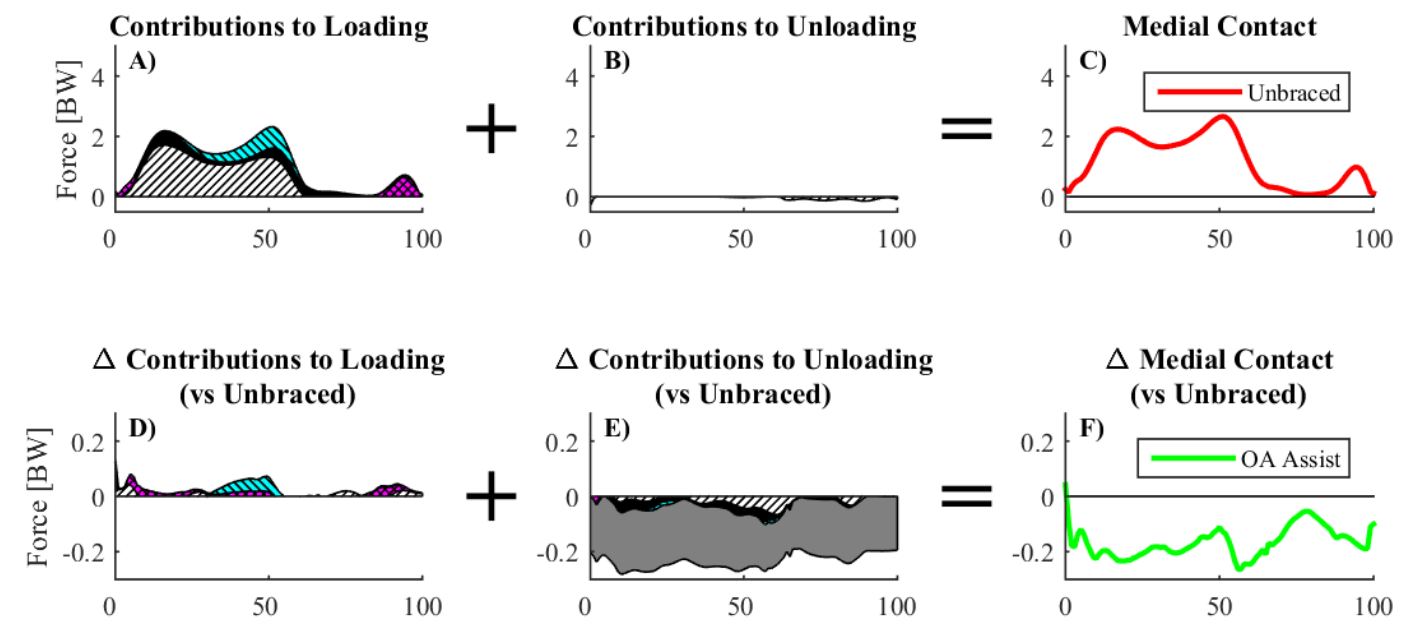

$\Delta$ Contributions to Unloading (vs Unbraced)
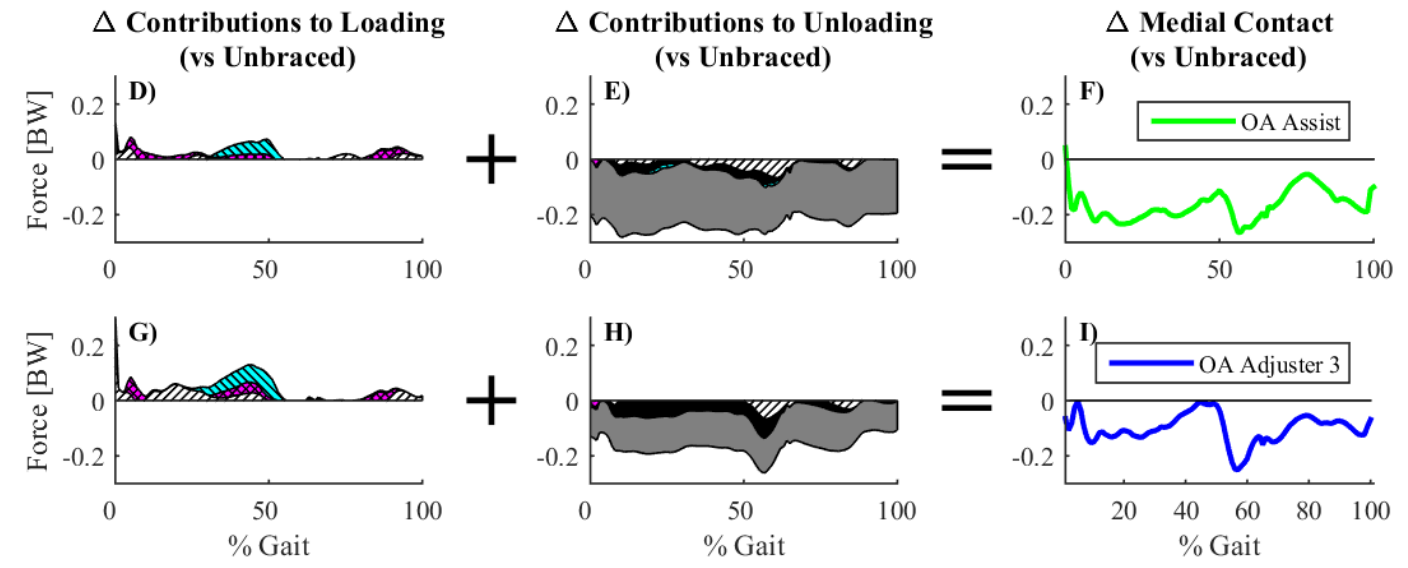

Wla $\mathrm{M}_{\text {adduction }}$ Quadriceps Hamstrings $\quad$ Gastrocnemii $\square$ Knee Brace

Figure 7). In literature, the effect of bracing on the external knee adduction moment (ignoring the load applied by a brace) is unclear; some studies found a reduction $[18,19,57]$ while others 
found no change $[12,58,59]$. In this study, there was no change in the knee adduction moment (
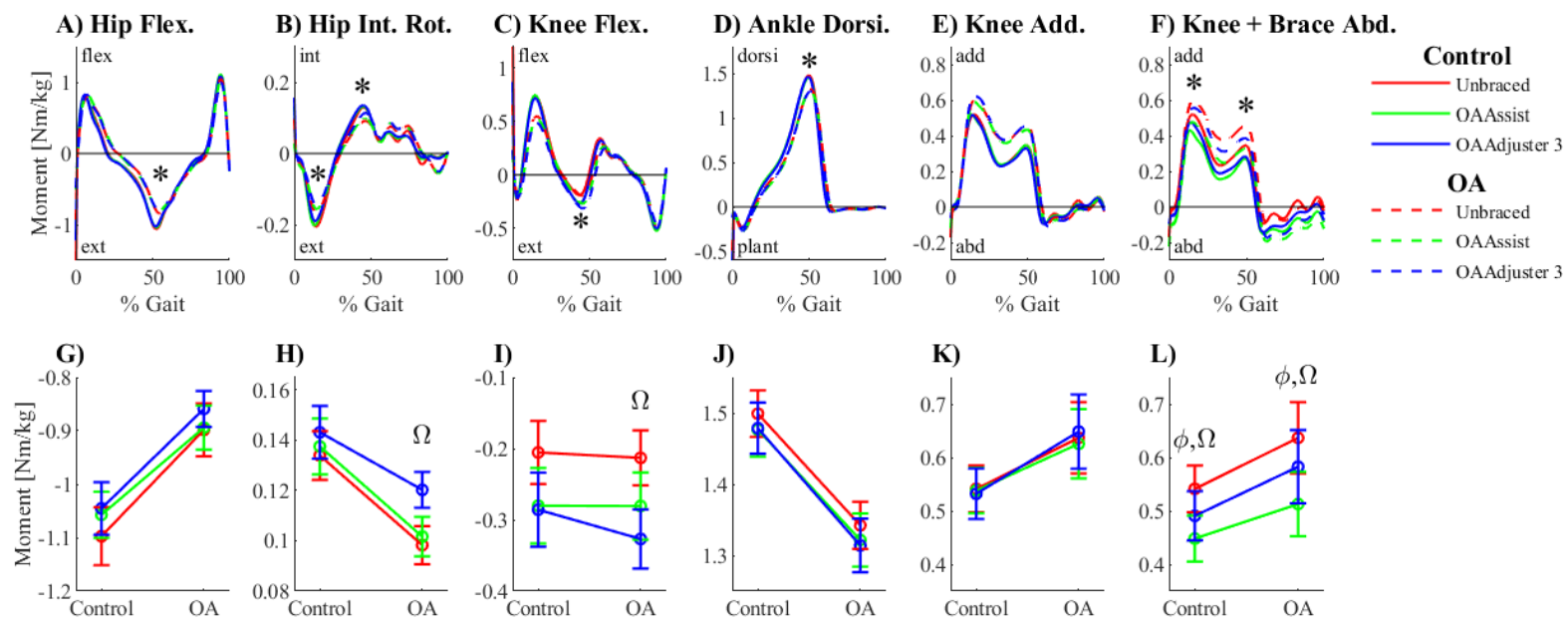

Figure 3E), and therefore a negligible contribution of the inverse dynamics load to changes in the medial and lateral knee contact forces due to bracing (
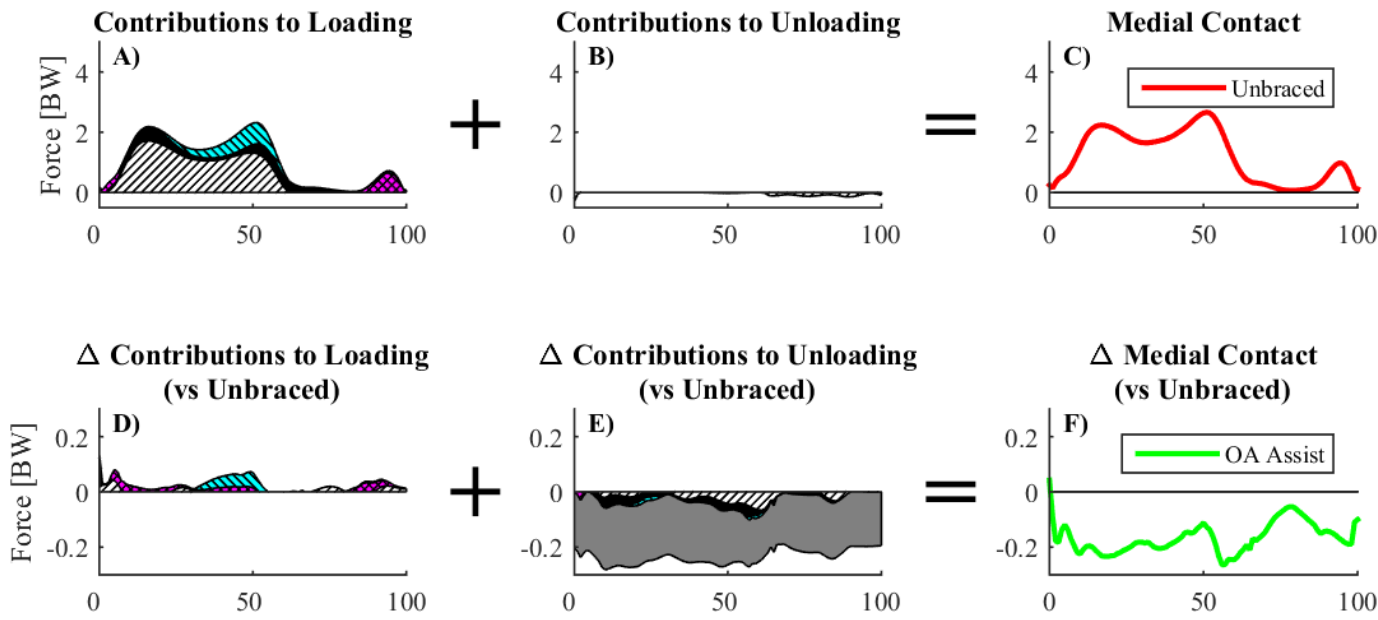

$\Delta$ Contributions to Unloading
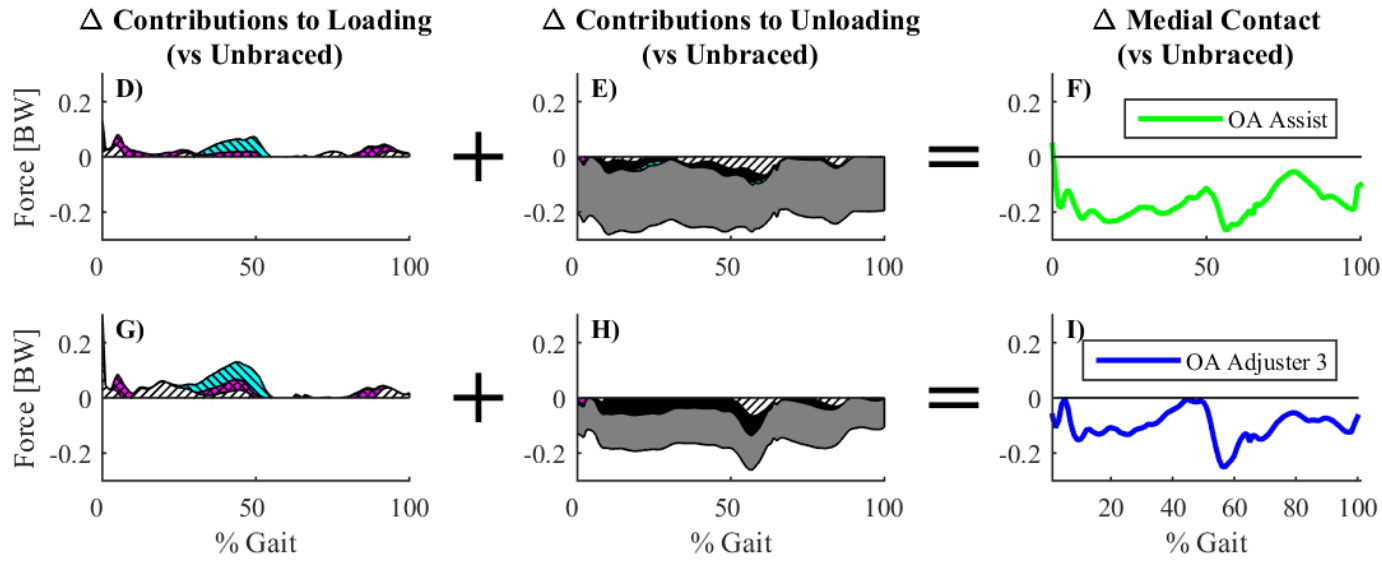

WIA $\mathrm{M}_{\text {adduction }}$ Quadriceps Hamstrings

Figure 7). Thus, both braces reduced medial contact loads without substantially changing gait dynamics ( 

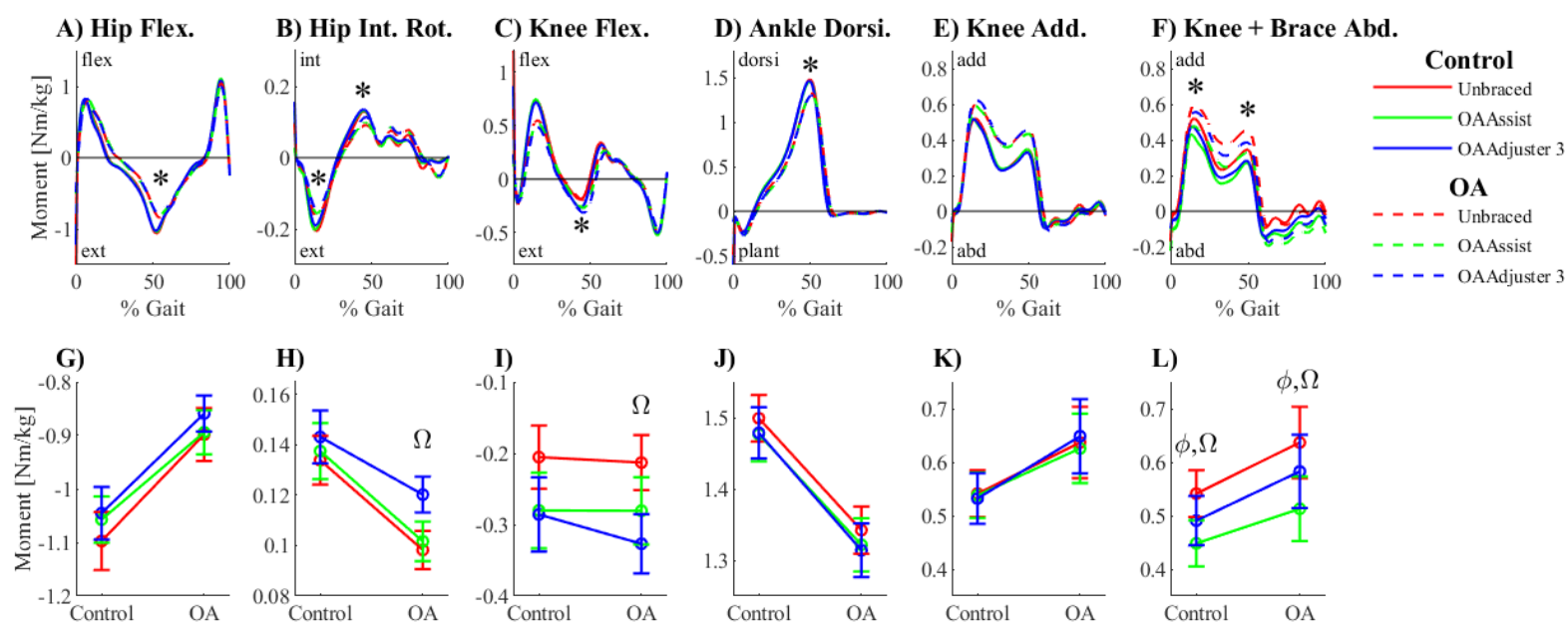

Figure 3) or muscle contributions to loading (
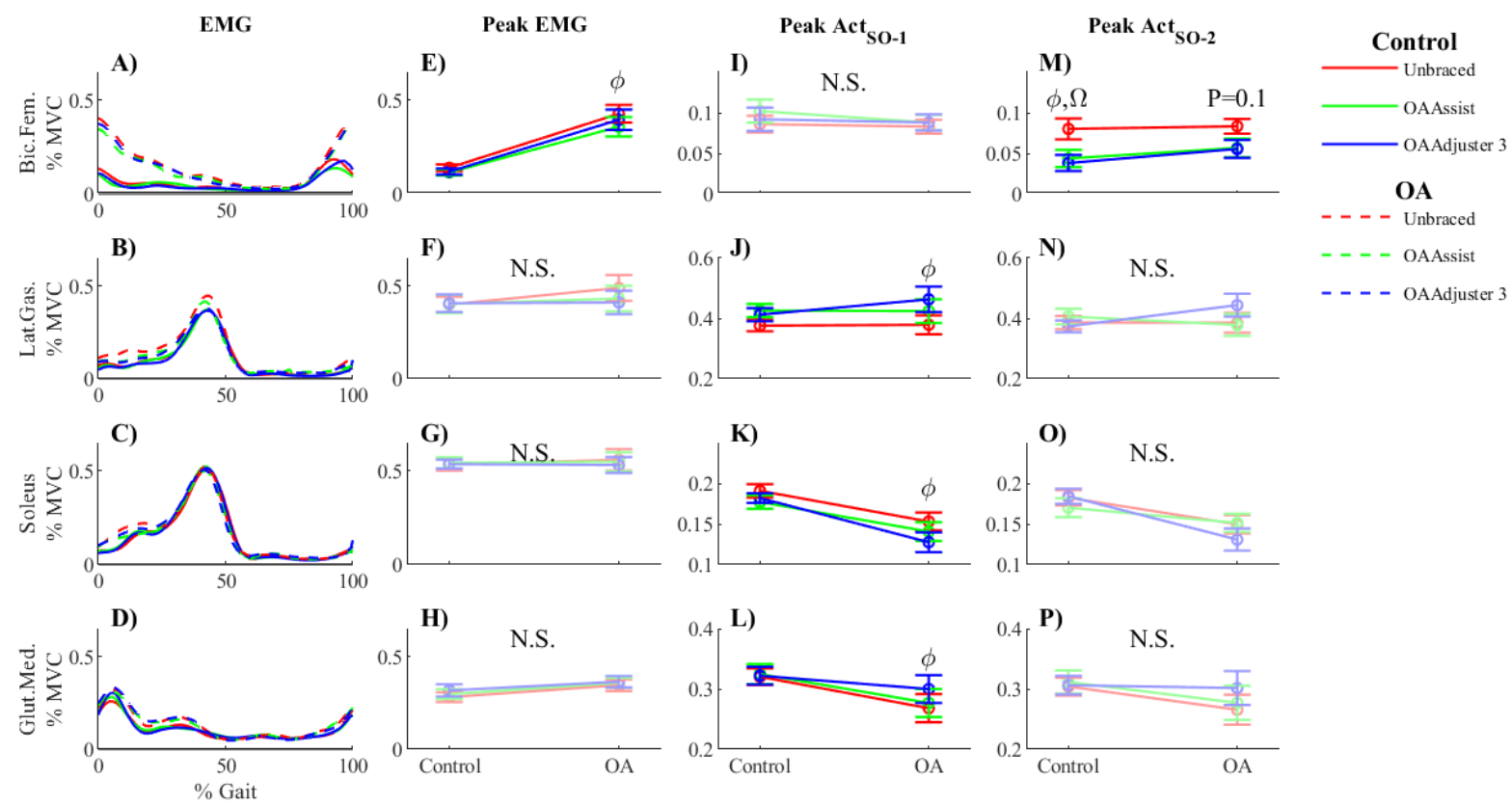

Figure 5, 

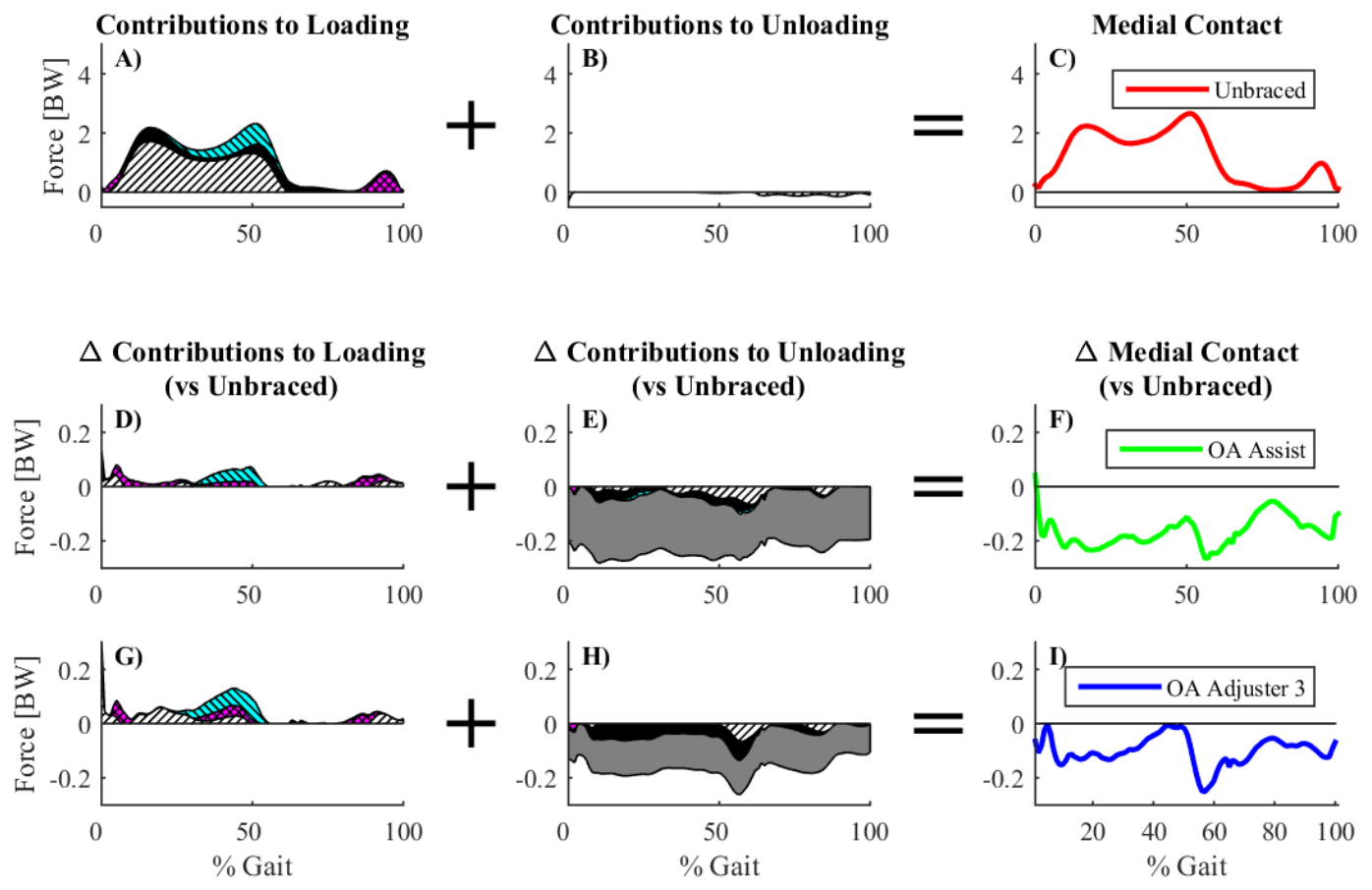

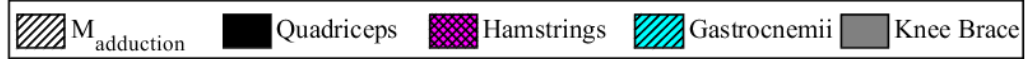

Figure 7). Based on these findings, it remains unclear why other studies $[7,10]$ have found such inter-individual variance in brace effectiveness. Future work should investigate whether subgroups exist with unique dynamics or muscle activation that were masked by group averaging. Indeed, we recently identified the external hip adduction moment as a potential "biomarker" to explain why some subjects achieve a greater medial load reduction [60]. 
A secondary objective of this study was to contrast the function of two different brace designs (

A) OA Assist

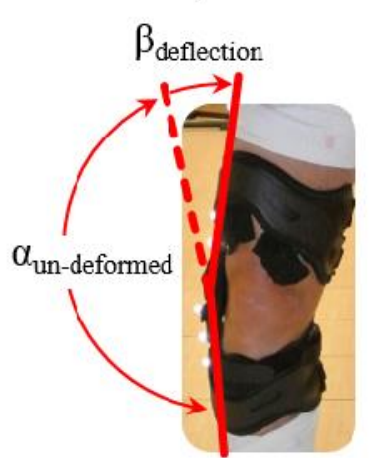

$\beta_{\text {deflection }}=\theta_{\text {meas }}$
B) OA Adjuster 3
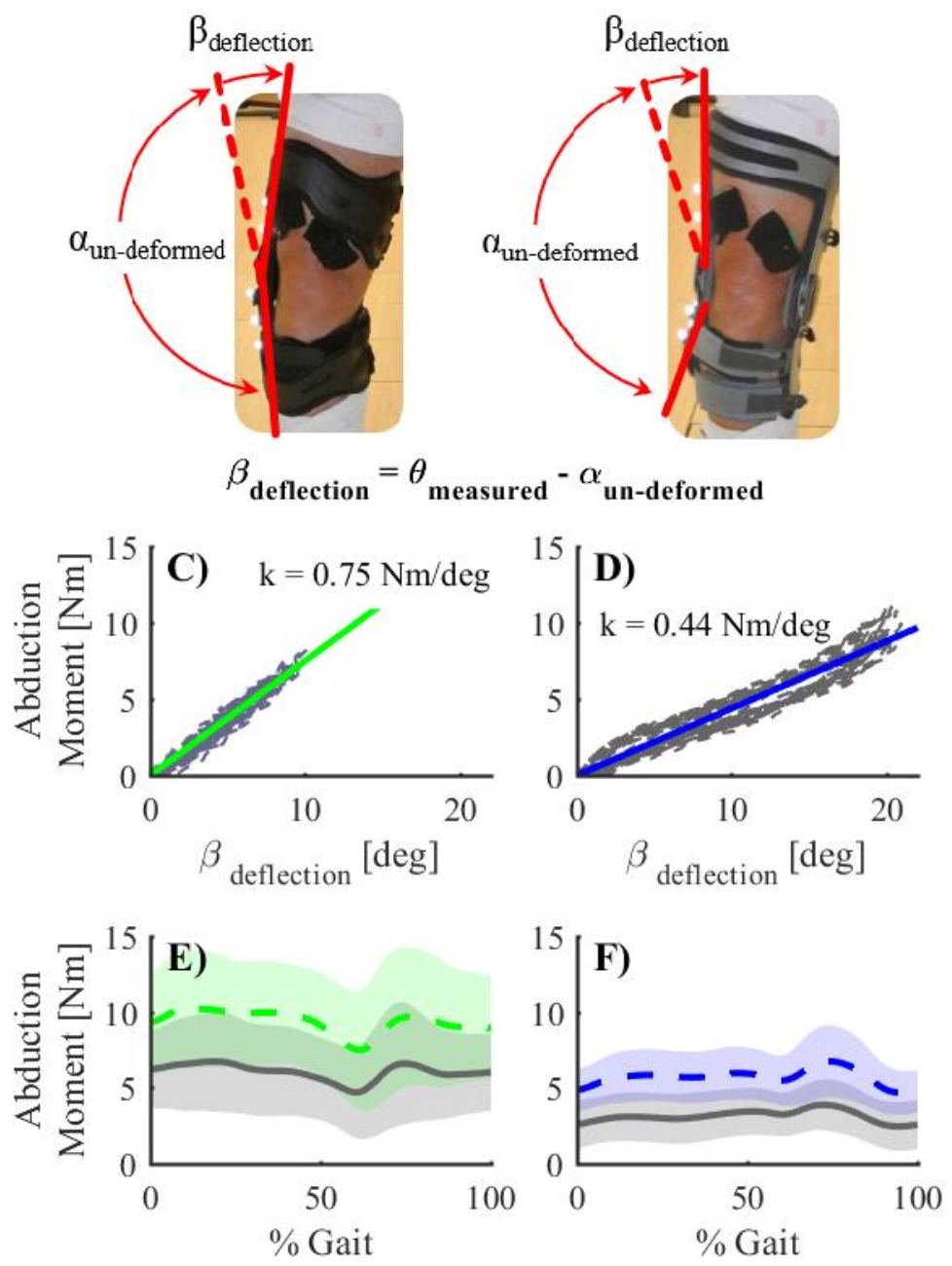

Figure 1). Previous studies have standardized brace loading by counting the number of 'turns' of the tightening screw $[10,17]$. In this study, brace deflection angles were measured dynamically, which allowed adjustment of each brace based on each participant's perceived comfort. Across all subjects, the single-beam OA Assist applied a greater unloading moment to the knee than the 
dual-beam OA Adjuster 3 brace (

A) OA Assist

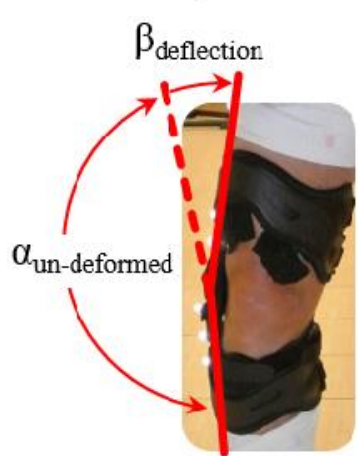

B) OA Adjuster 3
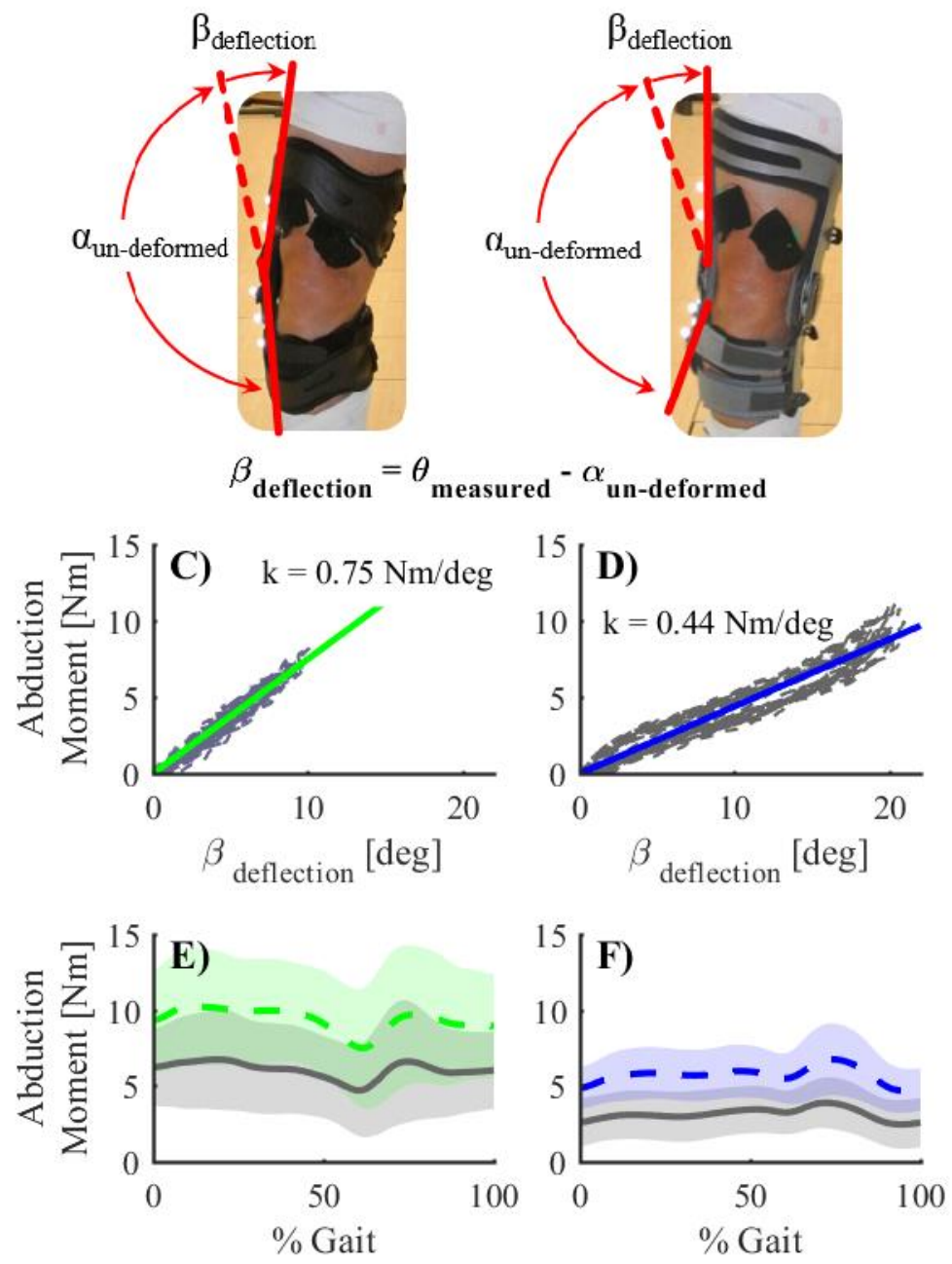
Figure 1, Table 2), which resulted in a greater reduction in medial loading (Table 3,
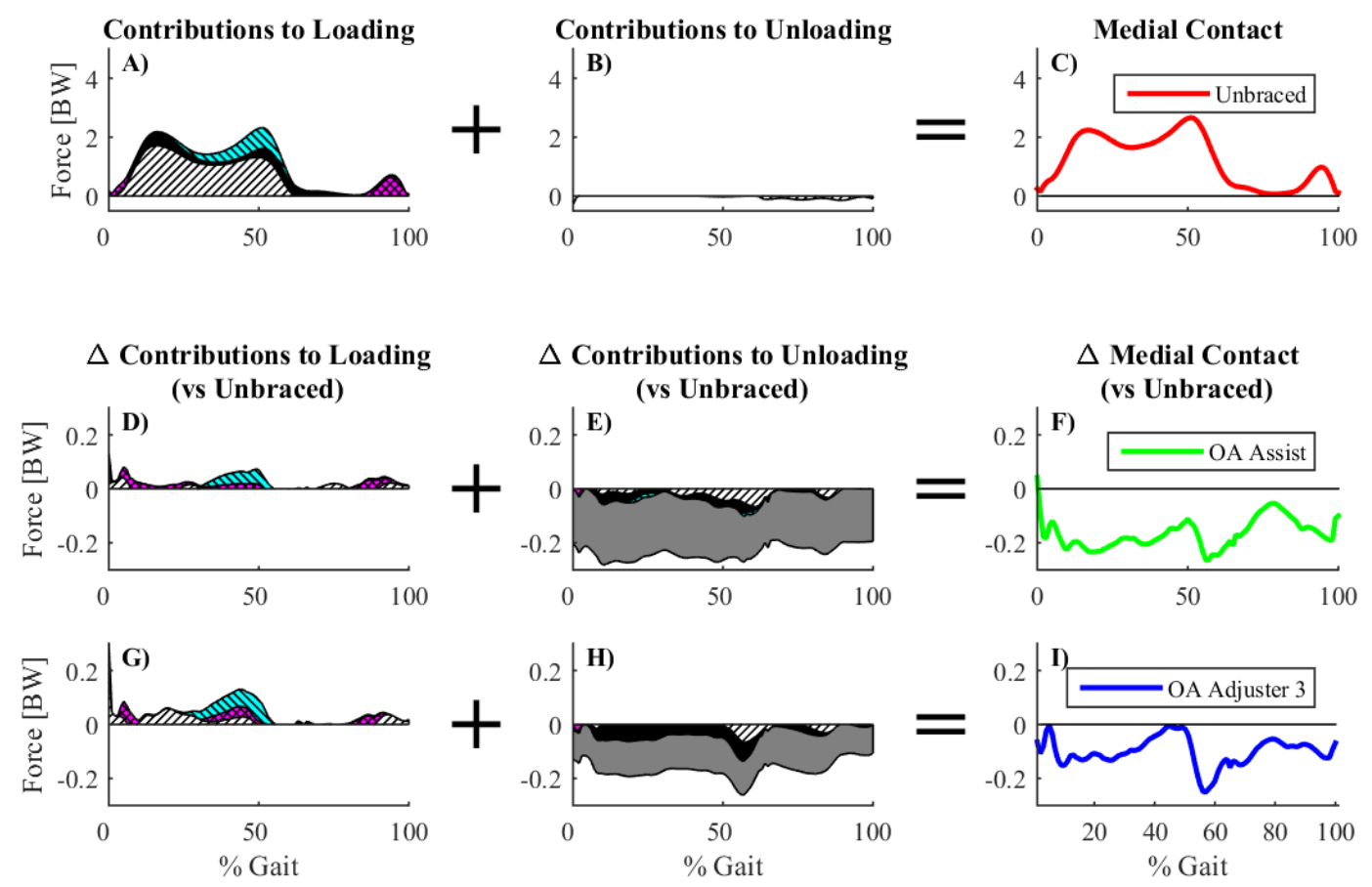

WIIA $\mathrm{M}_{\text {adduction }}$ Quadriceps Hamstrings 
Figure 7). However, both braces are capable of similar unloading moments (

A) OA Assist

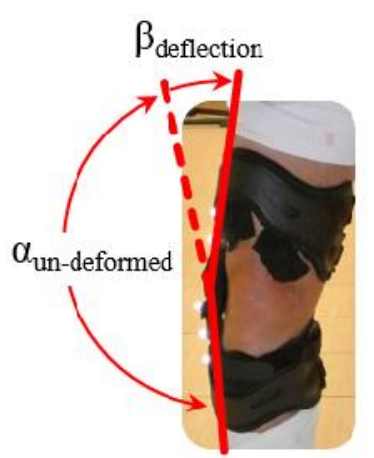

$\beta_{\text {deflection }}=\theta_{\text {meas }}$
B) OA Adjuster 3

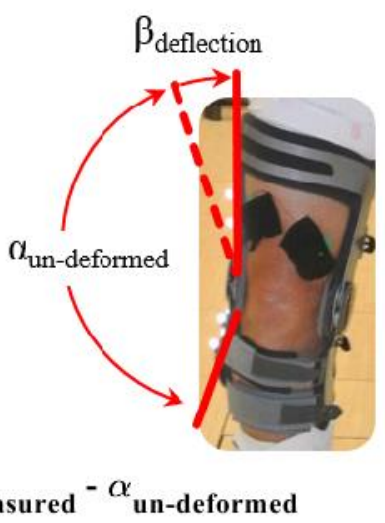

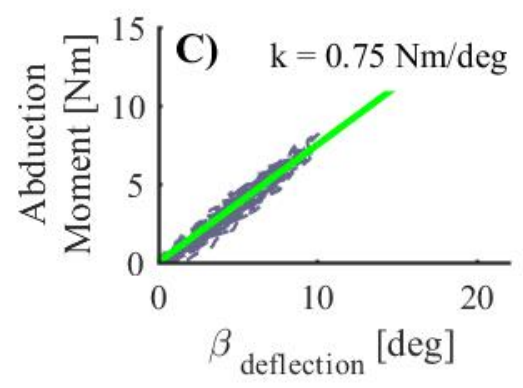
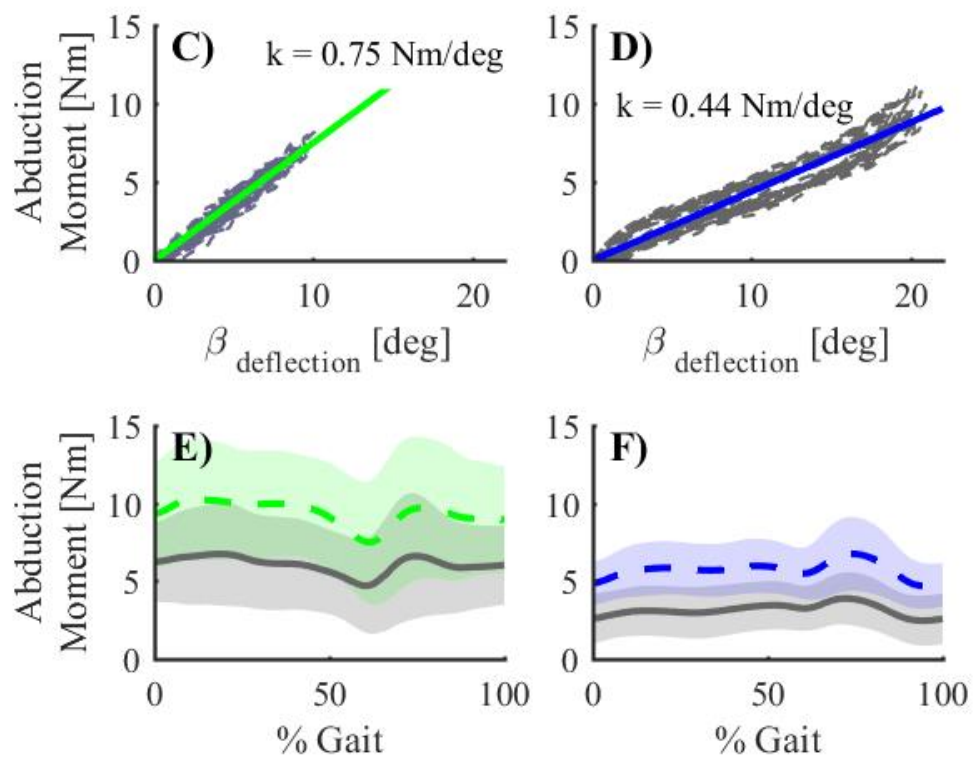

Figure 1), and it is unclear why subjects selected a larger moment with the OA Assist brace. We speculate that this may represent an acclimatization effect, since the OA Assist brace was always tested last to avoid dislodging sensors ( $\$ 2.4$ Procedure). Future work could employ pressure sensors to assess comfort, and should include both randomization and a longer acclimatization period to fully elucidate differences between braces.

A primary limitation of this study is the use of a static optimization model to estimate muscle forces. This type of model does not inherently capture changes in antagonist muscle co- 
contraction [61] as may occur with bracing [17] or knee osteoarthritis [21]. Indeed, we identified four muscles for which static optimization predictions did not match EMG measurements (
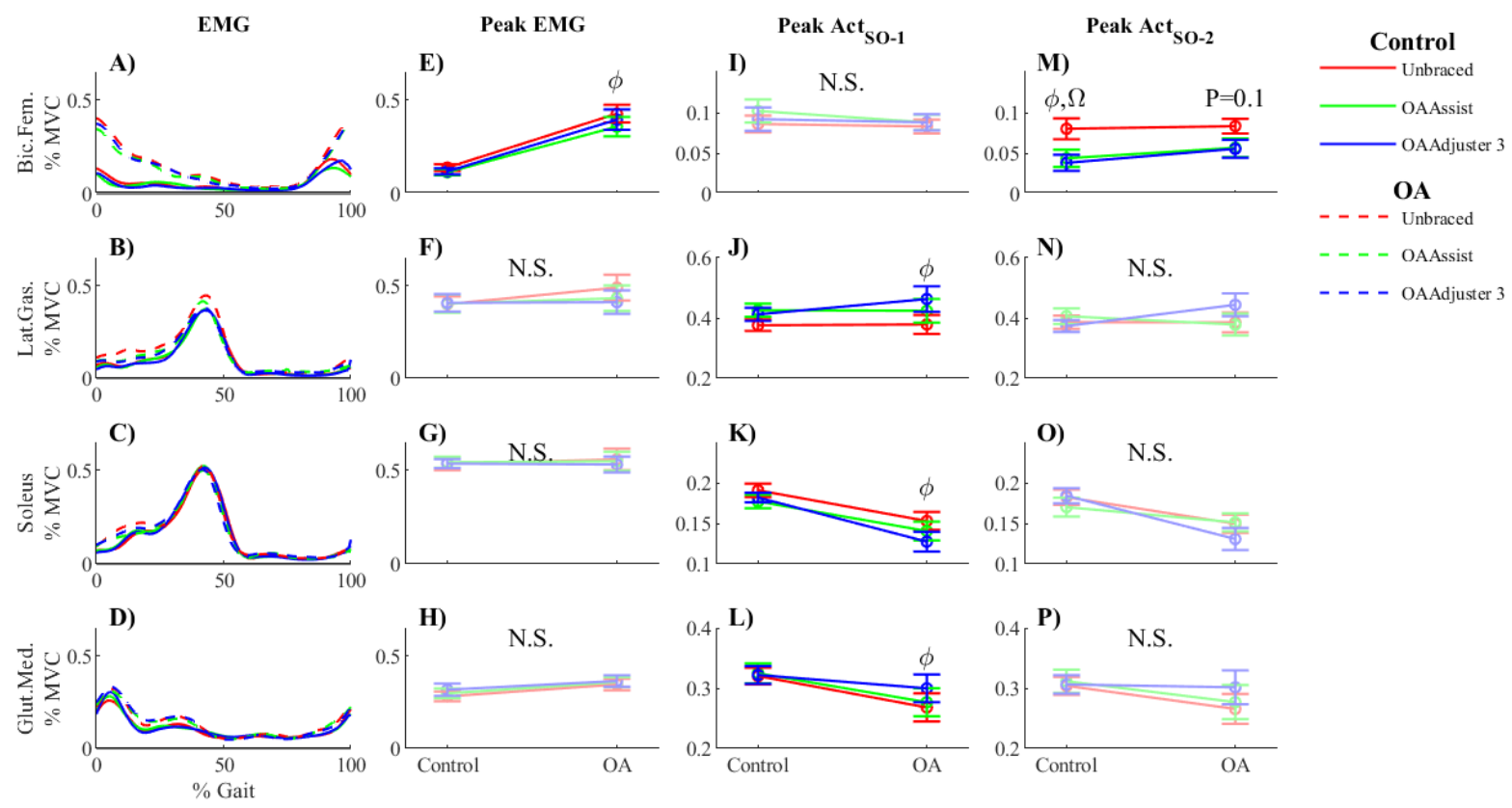

Figure 5). To assess whether these discrepancies confounded our results, we performed a follow-up simulation where the change in activation from unbraced to braced conditions was constrained to match measured EMG data (SO-2,
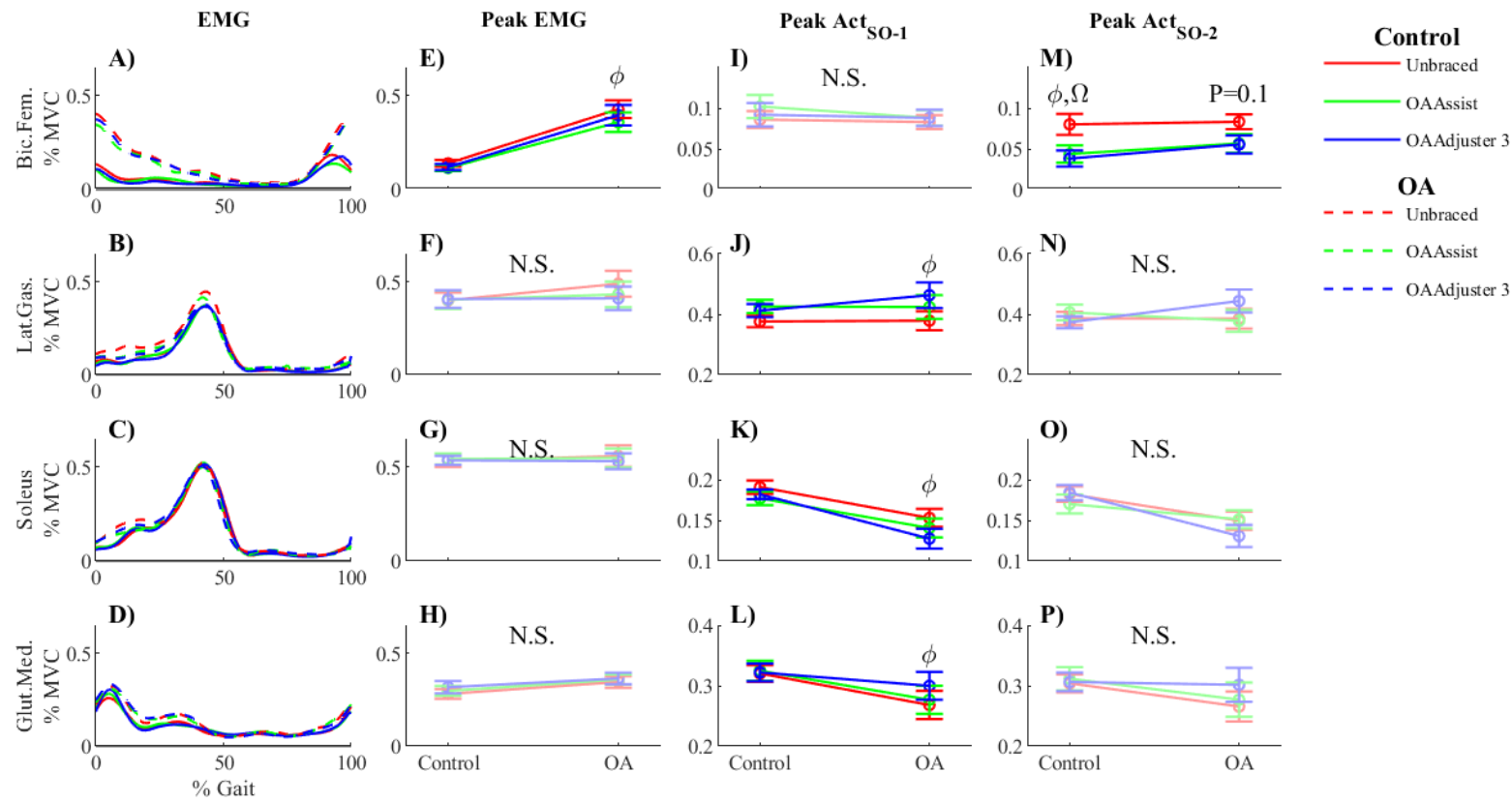
Figure 5, Supplementary Material). The change in medial loading did not differ between the original and follow-up simulations and both simulations, which supported our observation that the brace moment is the primary contributor to reducing medial knee loads ( 

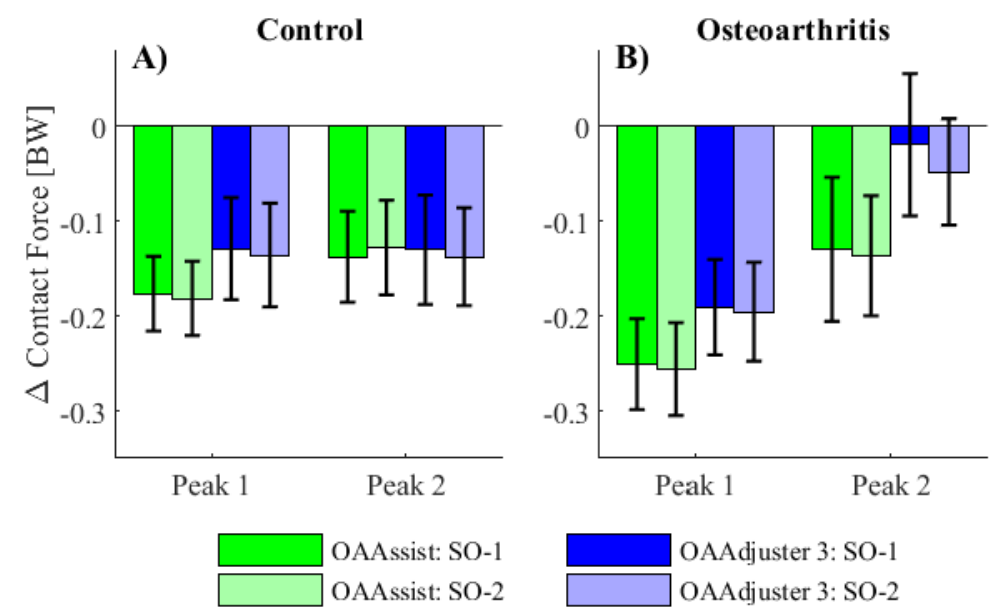

Figure 6, Supplementary Material). While an EMG-driven model is more suitable for capturing patient-specific muscle co-contraction [62], it is unlikely to have revealed different conclusions in the present study where mean changes in EMG were too small to significantly alter contact loads.

A second, related, limitation is the use of a 1-DOF knee joint model that does not predict changes in knee alignment or muscle activation due to knee abduction moments. Valgus bracing may reduce the knee adduction angle by approximately 1-2 degrees during gait [7], which could reduce medial contact load predictions by roughly $0.05-0.1 \mathrm{BW}$, or $2-4 \%$ [63]. Further, changes in knee adduction angles or moments may reduce strain in passive tissues (e.g. ligaments) and thus reduce contact forces while also altering muscle forces [64]. Indeed, mild co-contraction (10-20\%) of quadriceps and hamstrings has been observed during isolated ab/adduction loading [65], but it is unclear whether this is a neuromuscular strategy to resist abduction loads or an artifact of the overall coordination pattern (primarily hip muscle activation). Further, subjects exhibit a significant muscle reflex response to positional knee abduction perturbations $\left(+7^{\circ}\right.$ at $60^{\circ} / \mathrm{sec}, \sim 100 \mathrm{Nm}$ ), but it is unclear how this translates to dynamic movements [66]. Future work should investigate mechanisms to accurately predict changes in knee muscle activation due to 
dynamic abduction moment and/or angle perturbations; this requires knee models with additional DOF $[64,67,68]$. However, it is important to consider that EMG data did not reveal substantial differences between braced and unbraced conditions (
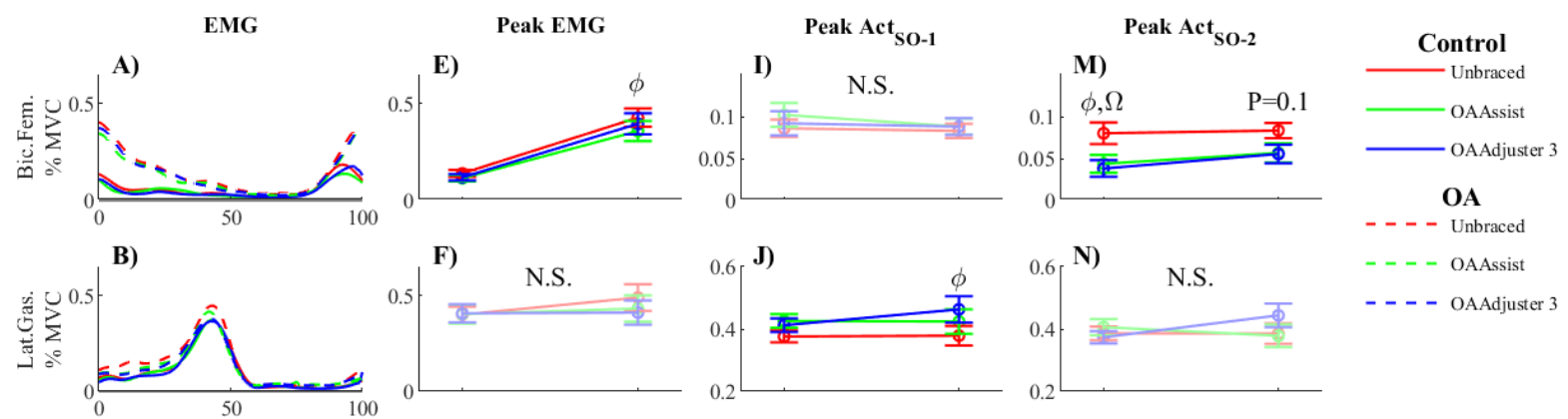

- - - OAAssist
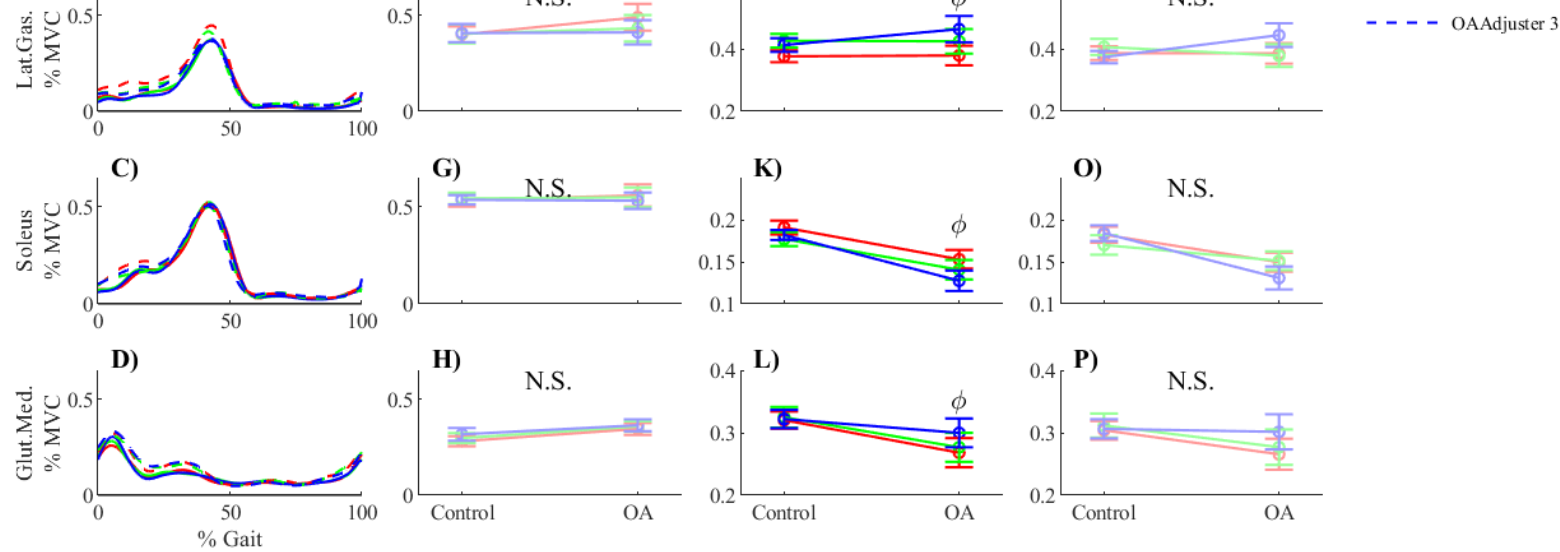

Figure 5); therefore the use of a 1-DOF knee joint is unlikely to have limited our ability to understand the primary mechanisms of unloader brace effectiveness.

Finally, it is important to note that maximal EMG amplitude was observed during MVIC trials in only $83 \%$ of muscles, across all subjects. It is widely known that isometric tasks, while generally repeatable [33], do not always yield maximal neuromuscular activation [38]. In this study, the use of a different normalisation approach would have changed the absolute magnitude of activation for $17 \%$ of muscles. Nevertheless, since normalization was constant across brace conditions, it is unlikely to have affected our primary conclusions about the relative importance of muscle forces for brace effectiveness. 
This study has important implications for both clinicians and brace designers. Firstly, while a previous study [17] suggested that braces with neutral alignment (i.e. zero abduction moment) perform as well as or better than braces with valgus alignment (i.e. applying an abduction moment) in reducing measures including pain and muscle co-contraction, the current study suggests that application of a brace abduction moment is crucial to reduce medial knee contact loads. Future work should investigate mechanisms to increase the brace abduction moment without discomfort, while considering the possible dissociation between reduction in pain and reduction in mechanical loading. Secondly, despite the dominance of the brace abduction moment effect, the current study reveals that even small changes in gait mechanics and muscle activation can enhance, or inhibit, brace effectiveness. For example, even without a significant change in EMG or muscle activation, a small decrease in late-stance hip and early-stance knee flexion moments (
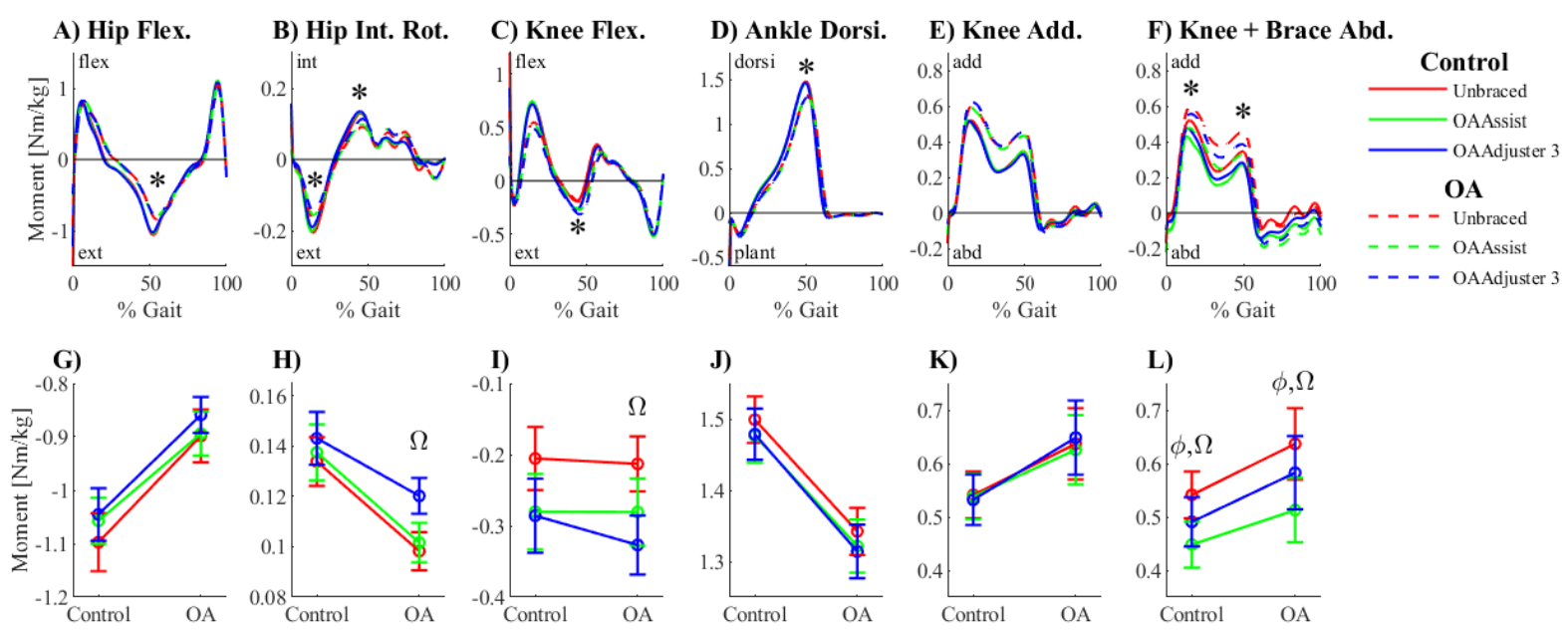

Figure 3), caused the model to predict a slight decrease in the quadriceps contribution to medial contact loads ( 

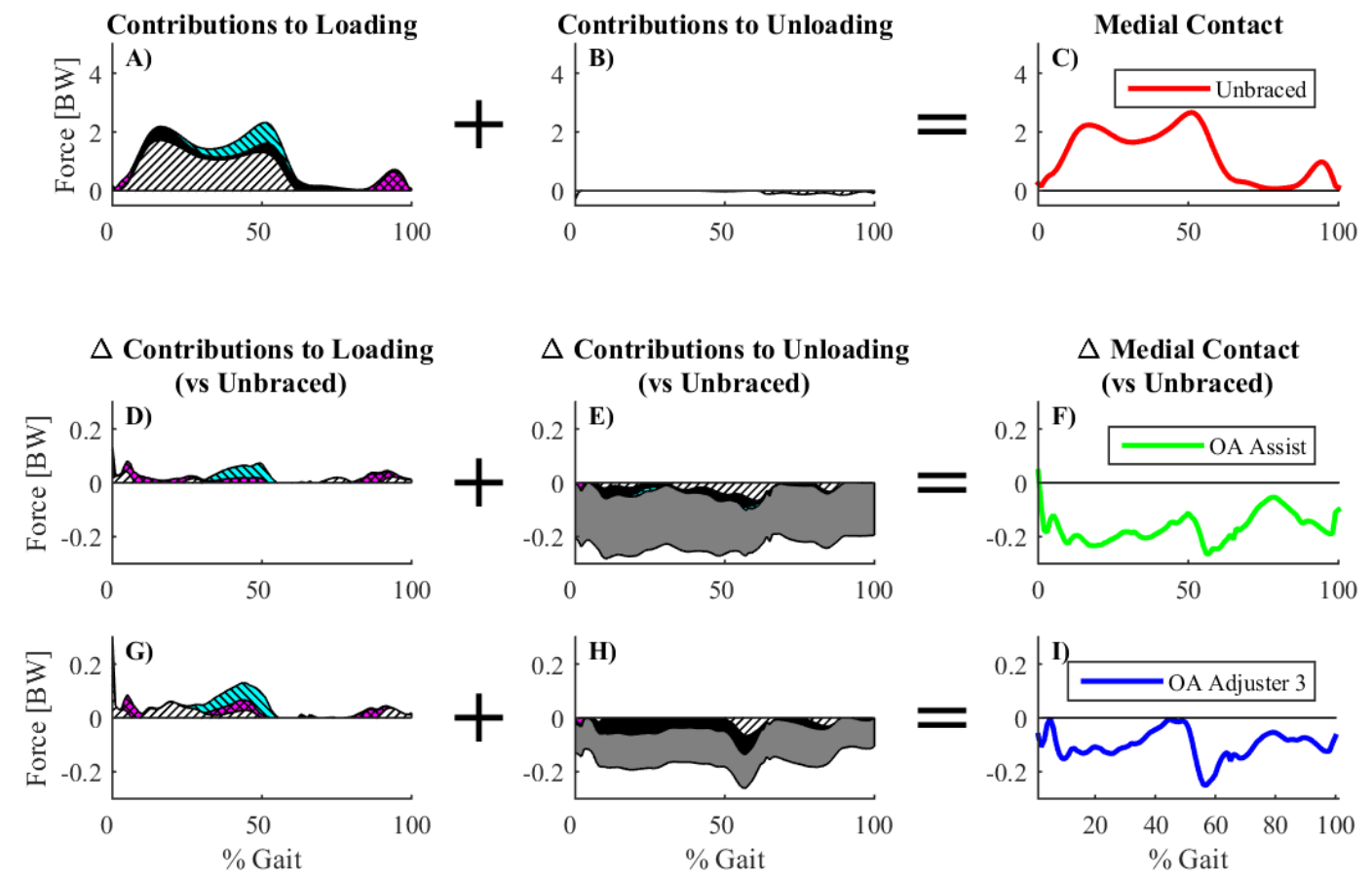

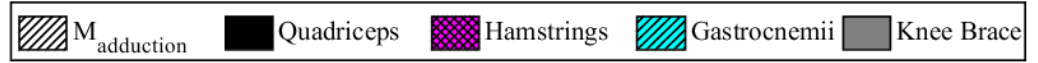

Figure 7) which enhanced medial load reduction. Conversely, a small increase in ankle moments
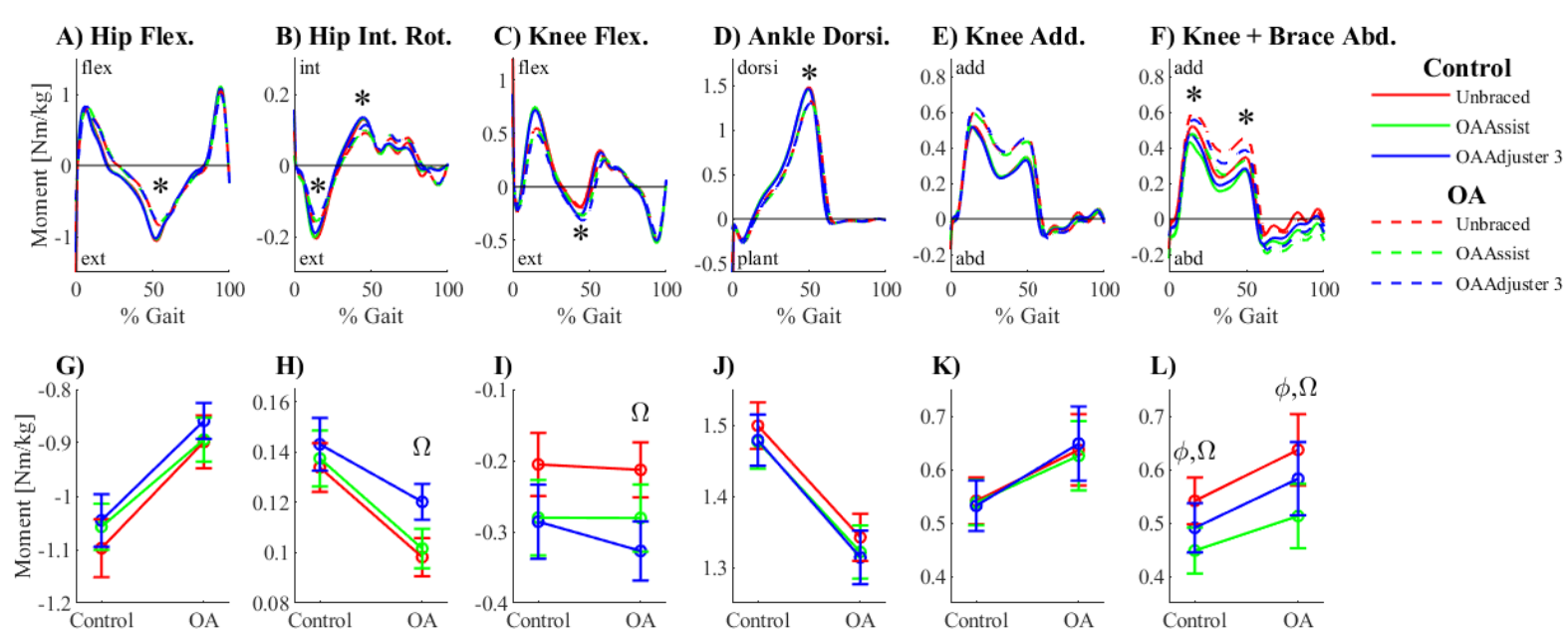

Figure 3D) caused an undesirable increase in gastrocnemii contributions to late-stance medial knee loads during braced conditions ( 

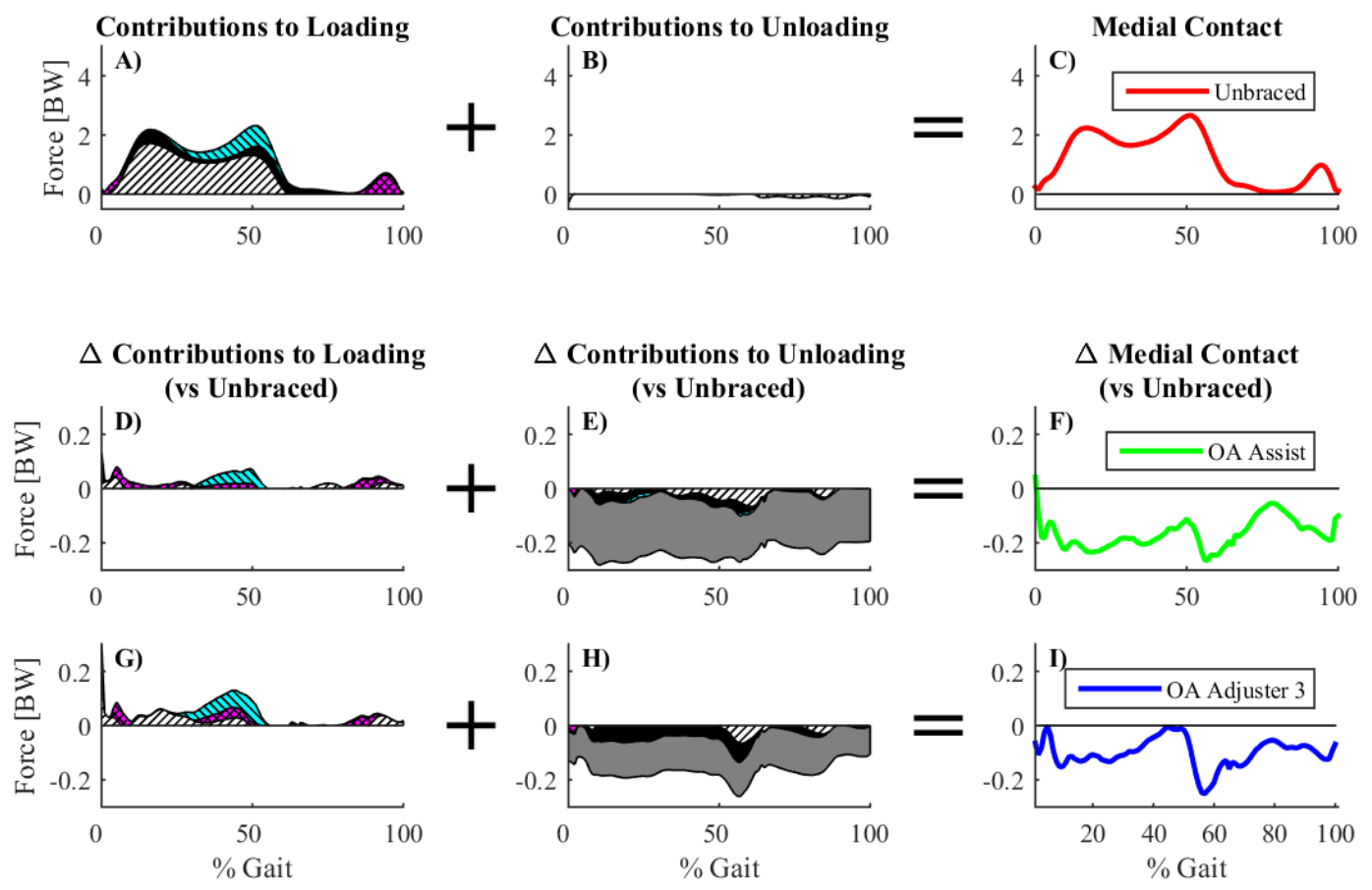

WIIA $\mathrm{M}_{\text {adduction }}$ Quadriceps Hamstrings $\mathbb{Z}^{\text {Gastrocnemii }} \square$ Knee Brace

Figure 7). These observations, which were consistent between both original (SO-1) and EMGinformed (SO-2) static optimization solutions, highlight the importance of considering both frontal and sagittal plane moments (the latter via muscle forces) in assessing knee contact loads [69]. Further, it appears that brace effectiveness might be enhanced by including mechanisms or rehabilitation training to modify both gait patterns and neuromuscular coordination [70].

In conclusion, a detailed musculoskeletal model revealed that knee unloader braces are effective in reducing medial knee loads primarily through the application of an external abduction moment. While these braces in this study were both from the same manufacturer (DJO Global, Vista, CA, USA), it is likely that the dominance of the applied abduction moment in reducing medial loads ( 

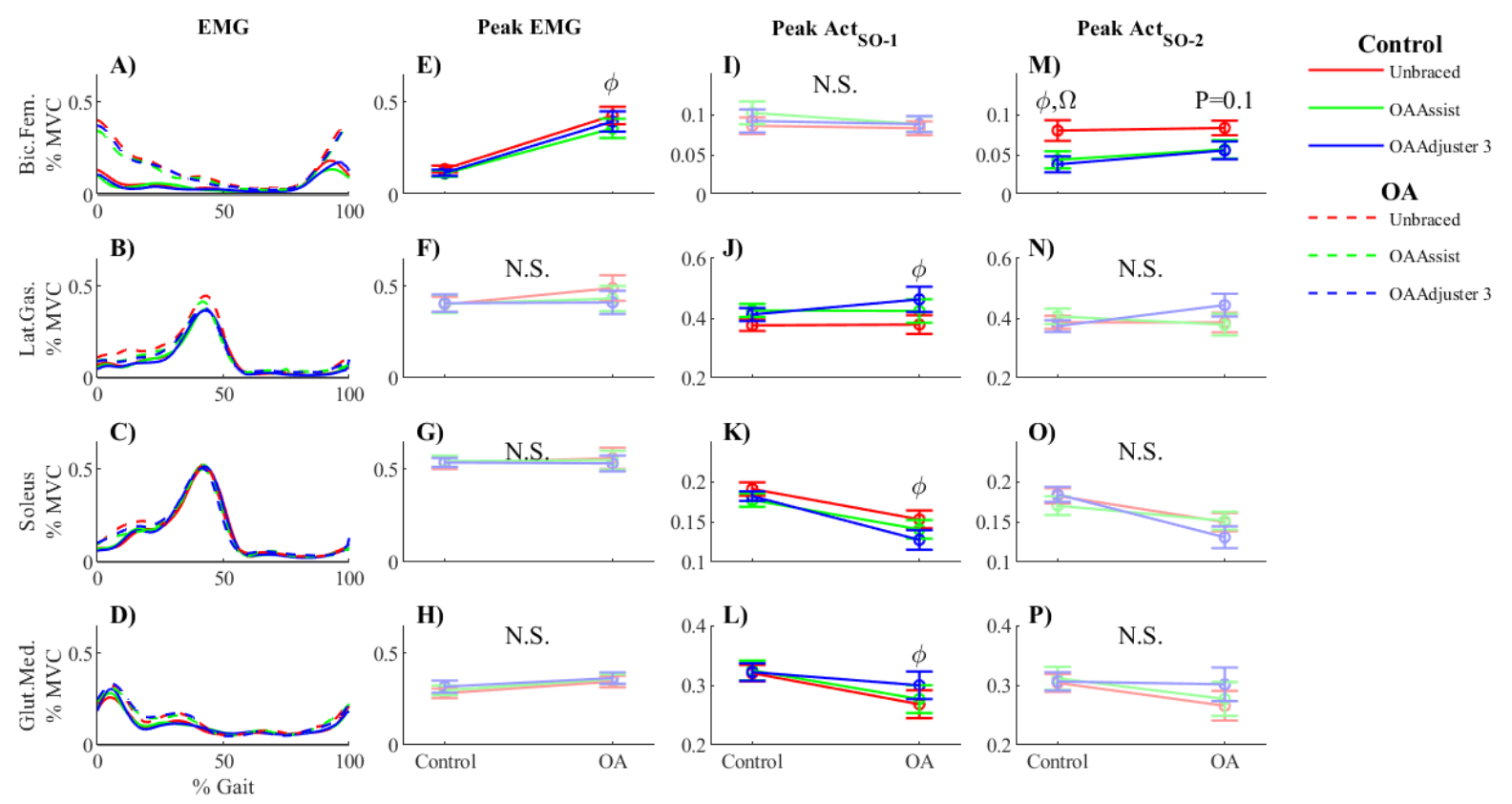

Figure 5) can be generalized to other unloader braces which apply the same principle of threepoint bending.

\section{Acknowledgements}

The sixteen knee braces used in this study were supplied by DJO Global Inc. This study was funded by NSERC.

\section{Conflict of Interest}

The authors have no conflict of interest to declare. 


\section{A) OA Assist}

\section{B) OA Adjuster 3}
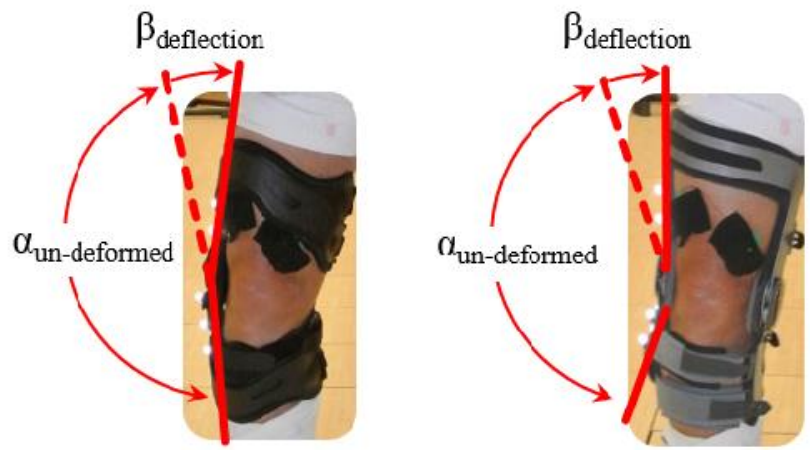

$$
\beta_{\text {deflection }}=\theta_{\text {measured }}-\alpha_{\text {un-deformed }}
$$
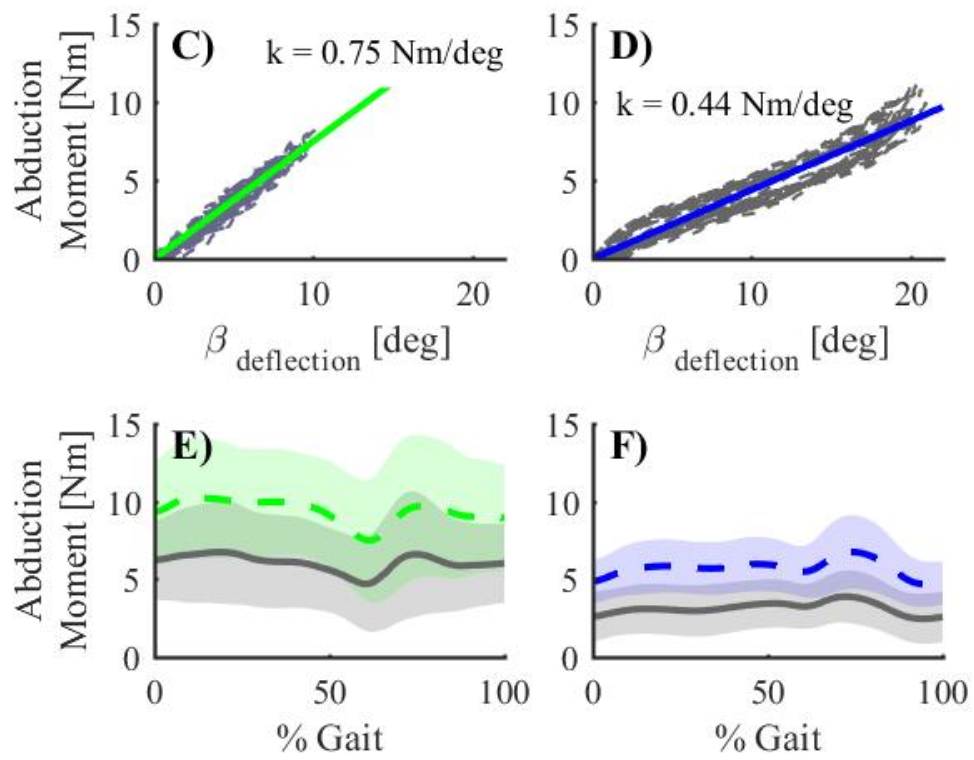

Figure 1: OA Assist (A) and OA Adjuster 3 (B) knee braces (DJO Global, Vista, CA, USA) used in this study, shown for example on a varus-aligned subject with medial knee osteoarthritis. EMG electrodes on the vasti are visible below distal to the brace strap, but proximal to the knee. Reflective markers, visible along the lateral beam of each brace, were used to compute brace

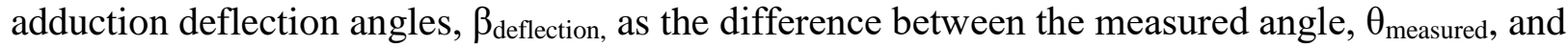
the known un-deformed angle, $\alpha_{\text {un-deformed. }}$ Braces were calibrated using a custom mechanical testing procedure to determine the linear relationship between brace adduction deflection angle, $\beta_{\text {deflection }}$ and the applied abduction moment. Dashed grey lines represent 16 load-unload cycles for each brace, the linear regression fit superimposed for OA Assist (solid green, C) and OA Adjuster 3 (solid blue, D) braces. Based on this calibrated stiffness, the abduction moment applied by the brace to each user's leg was computed throughout gait (E,F). Mean brace moments ( \pm shaded standard deviation) are shown for the control group (solid grey, E,F), and for the osteoarthritis group (dashed lines, E,F). For interpretation of the references to color in this figure legend, the reader is referred to the web version of this article. 

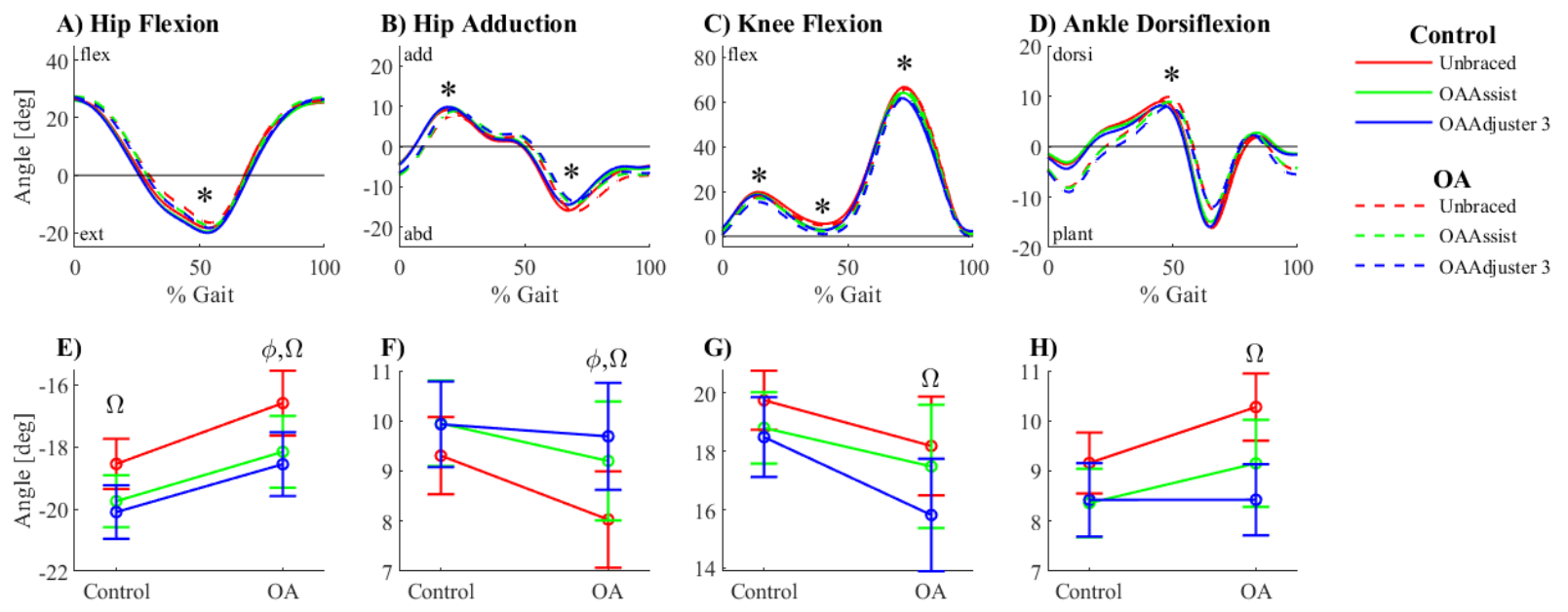

Figure 2: Mean joint angles for control (solid) and osteoarthritis (dashed) groups: hip flexion (A), hip adduction (B), knee flexion (C), and ankle dorsiflexion (D). OA Assist (green), and OA Adjuster 3 (blue) brace conditions were significantly different from the unbraced (red) condition for these angles $(*$, ANOVA $\mathrm{P}<0.05)$. An interaction plot $(\mathrm{E}-\mathrm{H})$ showing mean $\pm \mathrm{SEM}$ is presented for one of the significant peak measures from each waveform $(\mathrm{E}=$ hip flexion angle, $\mathrm{F}$ $=1^{\text {st }}$ peak hip adduction angle, $\mathrm{G}=1^{\text {st }}$ peak knee flexion angle, $\mathrm{H}=$ ankle dorsiflexion angle) . Significant pairwise comparisons $(\Phi=$ OAAssist vs unbraced, $\Omega=$ OAAdjuster 3 vs unbraced, $P$ $<0.05)$ between brace conditions were detected in both osteoarthritis and control groups for hip flexion angles, but only in the osteoarthritis subjects for hip adduction, knee flexion, and ankle dorsiflexion angles. For interpretation of the references to color in this figure legend, the reader is referred to the web version of this article. 

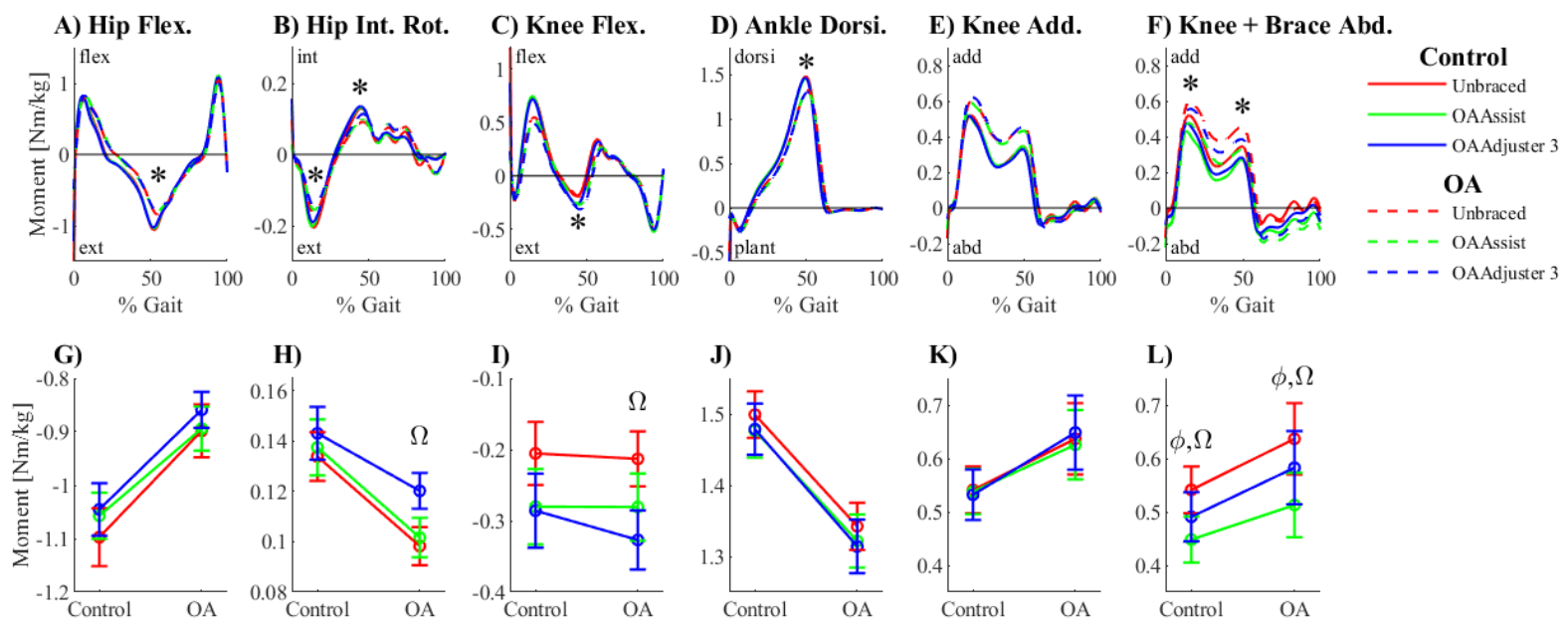

Figure 3: Mean joint moments for control (solid) and osteoarthritis (dashed) groups: hip flexion (A), hip internal rotation (B), knee flexion (C), ankle dorsiflexion (D), knee adduction (E, without including brace external moment) and net knee adduction ( $\mathrm{F}$, including brace external moment). OA Assist (green), and OA Adjuster 3 (blue) brace conditions were significantly different from the unbraced (red) condition for these moments $(*$, ANOVA P<0.05). An interaction plot $(\mathrm{G}-\mathrm{L})$ showing mean \pm SEM is presented for one of the significant peak measures from each waveform $\left(\mathrm{G}=2^{\text {nd }}\right.$ peak hip flexion moment, $\mathrm{H}=2^{\text {nd }}$ peak hip internal rotation moment, $\mathrm{I}=2^{\text {nd }}$ peak knee flexion moment, $\mathrm{J}=$ peak ankle dorsiflexion moment, $\mathrm{K}=$ knee adduction moment, $\mathrm{L}=2^{\text {nd }}$ peak net knee adduction moment including the brace abduction moment). Significant pairwise comparisons $(\Phi=$ OAAssist vs unbraced, $\Omega=$ OAAdjuster 3 vs unbraced, $\mathrm{P}<0.05)$ between brace conditions were detected in both osteoarthritis and control groups for the net knee adduction moment $(\mathrm{J})$, but only in the osteoarthritis subjects for hip internal rotation and knee flexion moments. Hip flexion and ankle dorsiflexion moments were not significant in post-hoc tests. For interpretation of the references to color in this figure legend, the reader is referred to the web version of this article. 

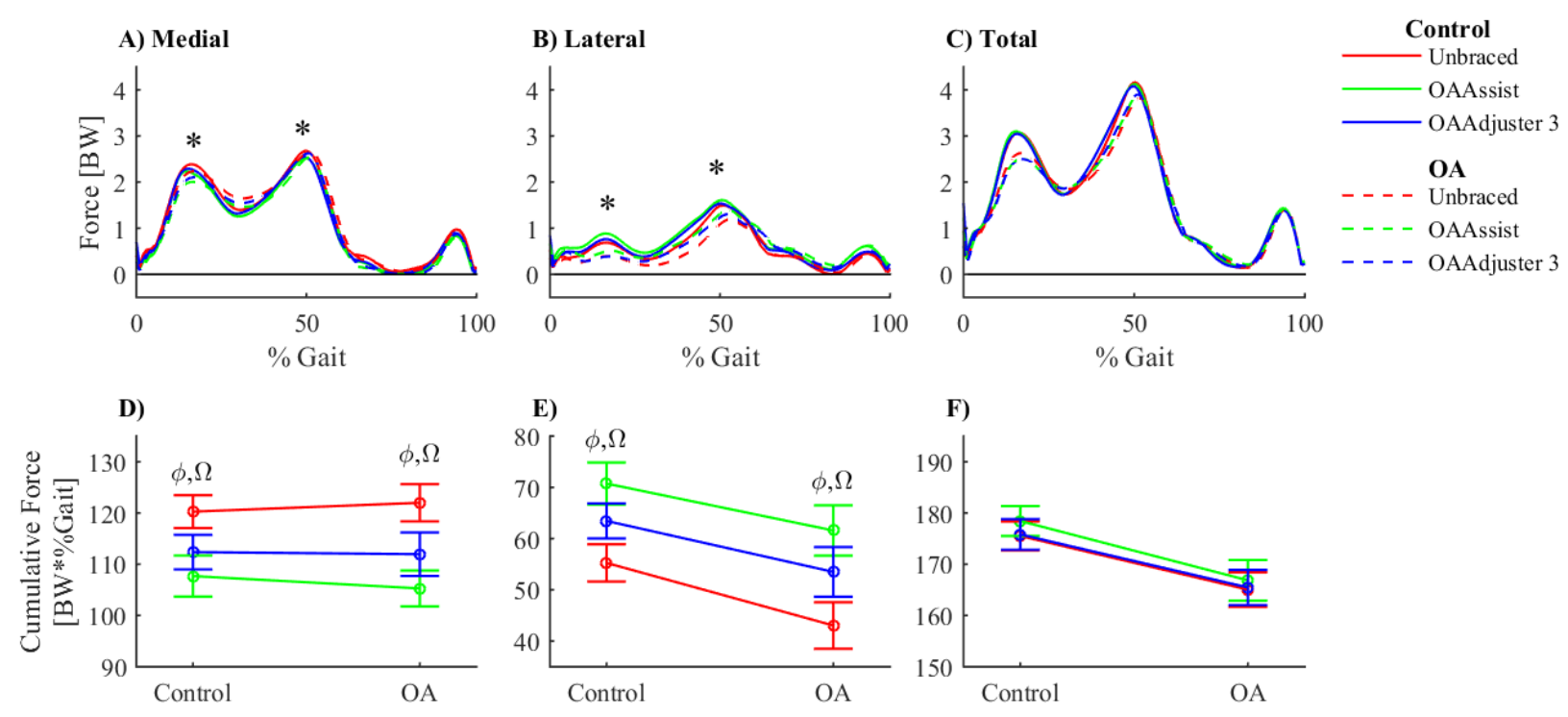

Figure 4: Mean medial (A), lateral (B), and total (C) knee contact forces for control (solid) and osteoarthritis (dashed) subjects during overground gait. OA Assist (green), and OA Adjuster 3 (blue) brace conditions were significantly different from the unbraced (red) condition for these contact forces $(*, \mathrm{P}<0.05)$.Interaction plots show mean $+-\mathrm{SEM}$ predictions of the cumulative medial (D), lateral (E), and total knee contact force $(\mathrm{F})$, integrated throughout the gait cycle $(\Phi=$ OAAssist vs unbraced, $\Omega=$ OAAdjuster 3 vs unbraced, $\mathrm{P}<0.05)$. Medial loads (A,D) decreased for both OA Assist (green) and OA Adjuster 3 (blue) conditions, as compared with the unbraced condition (red), while lateral loads increased due to bracing (B,E). Combined, there was no change in the integral of the total knee contact force due to brace use $(\mathrm{F})$, although a small significant difference was detected atthe first peak (C). For interpretation of the references to color in this figure legend, the reader is referred to the web version of this article. 

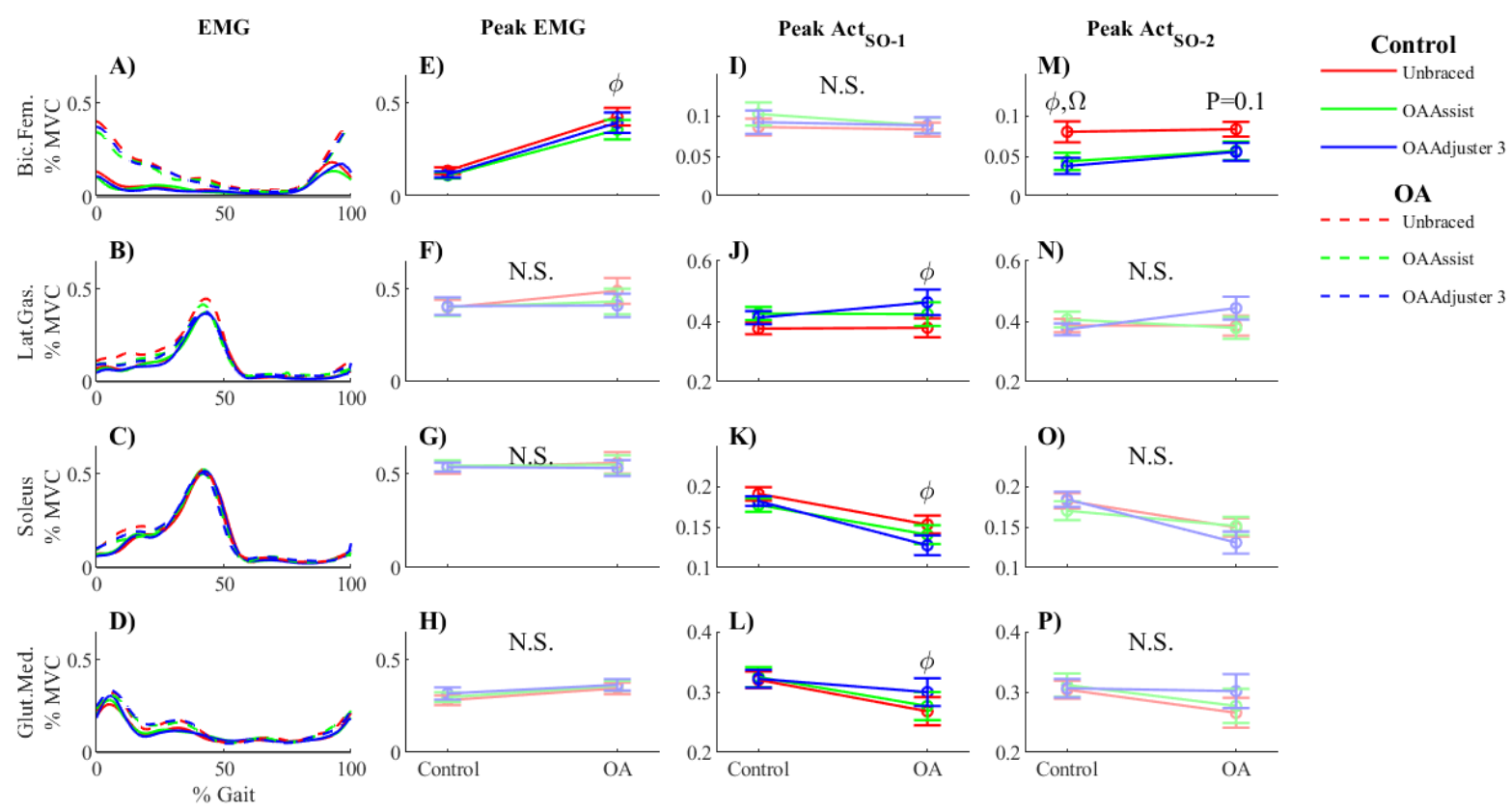

Figure 5: Mean enveloped EMG (A-D), peak EMG (E-H), peak activation from the first static optimization (SO-1, I-L), and peak activation from the second, constrained static optimization (SO-2, M-P). Data are presented for the four muscles for which the change in EMG due to bracing did not match the change predicted by simple static optimization (E-H vs I-L). Row 1 (A,E,I,M) shows biceps femoris; there was a decrease in peak EMG due to bracing (A,I), no change in the first optimization solution (I), and therefore the model was constrained to induce a decrease in the second optimization solution (M) in order to match the EMG. Rows 2-4 show lateral gastrocnemius, soleus, and gluteus medius, respectively. These muscles showed no change in EMG due to bracing, but a significant change was predicted in the original static optimization solution (F-H vs J-L). Consequently, these muscles were constrained to yield no significant change for the second, constrained, optimization solution (N-P). $(\Phi=$ OAAssist vs Unbraced, $\Omega=$ OAAdjuster 3 vs Unbraced, $\mathrm{P}<0.05, \mathrm{NS}=$ not significant) 

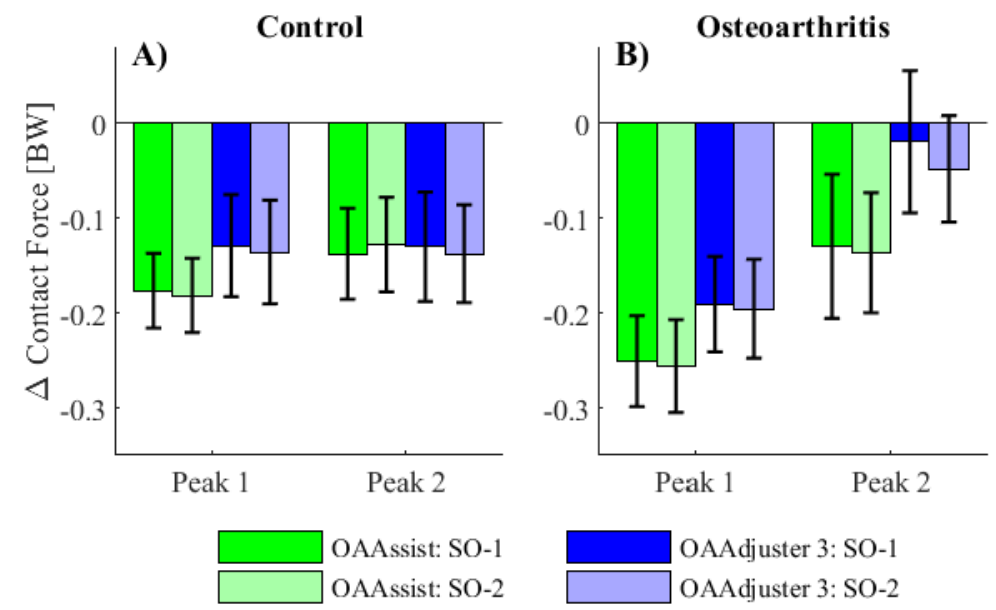

Figure 6: Mean \pm SEM change in peak medial knee contact force due to the use of OA Assist (blue) and OA Adjuster 3 (green) knee braces for control (A) and osteoarthritis (B) groups. The reduction in predicted medial contact force was not significantly different $(\mathrm{P}>0.76)$ between the original static optimization (SO-1, dark green, dark blue) and the follow-up constrained static optimization (SO-2, light green, light blue) in which estimated muscles activations were constrained to match observed changes in EMG. 

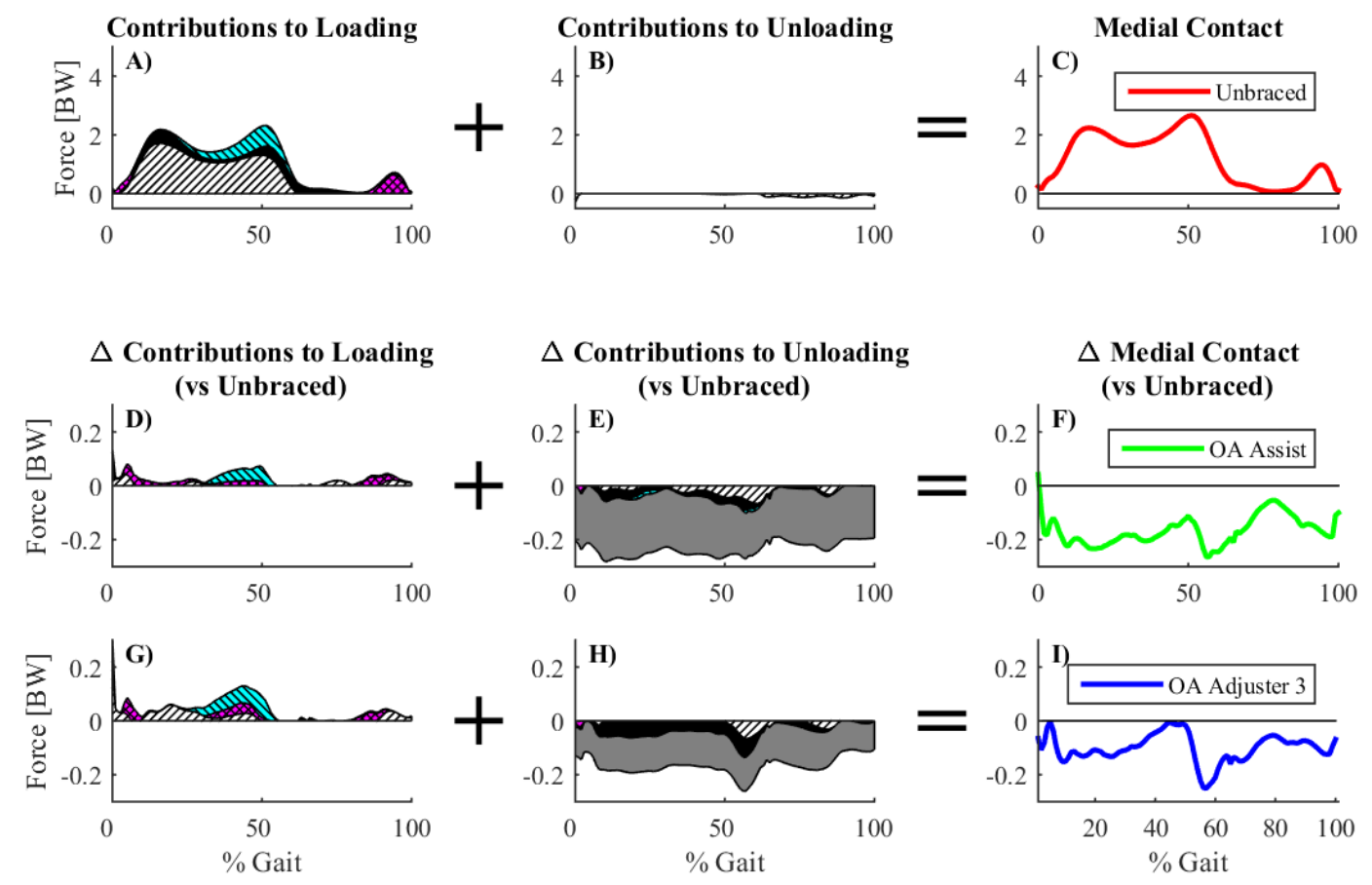

$\mathbb{Z} \mathrm{M}_{\text {adduction }} \quad$ Quadriceps Hamstrings $\mathbb{Z}$ Gastrocnemii $\square$ Knee Brace

Figure 7: Contributions of the knee adduction moment, muscle forces, and the knee brace abduction moment to unbraced medial knee contact forces (A-C), and to the change in medial loading when using the OA Assist (D-F) and OA Adjuster 3 (G-I) knee braces. These contributions are shown for the osteoarthritis group, as estimated using the original static optimization solution (SO-1). Loads that generate a positive (adduction) moment about the lateral tibial location act to increase medial knee contact force (first column, A,D,G), while loads that generate a negative (abduction) moment contribute to unloading the medial compartment (second column, B,E,H). The third column shows the sum of positive and negative contributions, which yields the medial knee contact force $(\mathrm{C}$, red), and the change in contact force due to the OA Assist (F, green) and OA Adjuster 3 (I, blue) braces. The adduction moment (primarily due to the ground reaction force passing medial to the knee) was the primary contributor to medial knee loading, while quadriceps and gastrocnemii made a smaller contribution (A). For each of the braced conditions, the abduction moment applied by the knee brace (E,H, shaded grey) was the primary contributor to reduced medial contact forces. Relative to the unbraced condition, the gastrocnemii and hamstrings muscles were estimated to increase their contribution to medial knee loads $(\mathrm{D}, \mathrm{G})$, while quadriceps contributions decreased $(\mathrm{E}, \mathrm{H})$. The total reduction in medial knee contact due to bracing was between 0.1 and $0.2 \mathrm{BW}$ throughout most of the gait cycle, for both brace designs (F,I). 


\section{Tables}

Table 1: Participant Characteristics, Mean (SD)

\begin{tabular}{lccc}
\hline Parameter & Osteoarthritis & Control & P-value \\
\hline Male & 9 & 8 & - \\
Female & 8 & 10 & - \\
Age [years] & $54(4)$ & $47(11)$ & 0.01 \\
Height [m] & $1.72(0.10)$ & $1.72(0.09)$ & 0.89 \\
Mass [kg] & $89(18)$ & $75(13)$ & 0.05 \\
BMI [kg/m ${ }^{2}$ ] & $30(4)$ & $26(3)$ & $<0.01$ \\
HKA [deg, $+{ }^{\text {ve }}$ varus] & $6.9(5.6)$ & $1.2(2.1)$ & $<0.01$ \\
Medial Joint Space [mm] & $2.1(1.8)$ & $5.1(0.9)$ & $<0.01$ \\
WOMAC - Pain & $66(18)$ & $100(1)$ & $<0.01$ \\
WOMAC - Stiffness & $57(22)$ & $99(6)$ & $<0.01$ \\
WOMAC - Function & $62(19)$ & $100(1)$ & $<0.01$ \\
WOMAC - Total & $62(18)$ & $100(1)$ & $<0.01$ \\
Gait Speed [m/s] & $1.3(0.1)$ & $1.5(0.2)$ & $<0.01$
\end{tabular}


Table 2: Unloading brace abduction moment applied to the knee during gait, Mean (SD) reported across all subjects

\begin{tabular}{lccl} 
& $\begin{array}{c}\text { Control } \\
\text { Mean } \\
(\mathrm{SD})\end{array}$ & $\begin{array}{c}\text { OA } \\
\text { Mean } \\
(\mathrm{SD})\end{array}$ & $\begin{array}{c}\text { P-value } \\
\text { (groups) }\end{array}$ \\
\hline OA Assist $[\mathrm{Nm}]$ & $6(3)$ & $9(4)$ & $<0.01$ \\
OA Adjuster $3[\mathrm{Nm}]$ & $3(2)$ & $6(2)$ & $<0.01$ \\
P-value (braces) & $<0.01$ & $<0.01$ &
\end{tabular}


Table 3: Change in contact loads due to bracing, Mean (SD)

\begin{tabular}{|c|c|c|c|c|c|c|}
\hline & \multicolumn{3}{|c|}{ Medial } & \multicolumn{3}{|c|}{ Lateral } \\
\hline & $\begin{array}{c}\text { Peak 1 } \\
{[\mathrm{BW}]}\end{array}$ & $\begin{array}{c}\text { Peak } 2 \\
\text { [BW] }\end{array}$ & $\begin{array}{c}\text { Integral } \\
{[\mathrm{BW} * \% \text { Gait }]}\end{array}$ & $\begin{array}{c}\text { Peak 1 } \\
{[\mathrm{BW}]}\end{array}$ & $\begin{array}{l}\text { Peak } 2 \\
{[\mathrm{BW}]}\end{array}$ & $\begin{array}{c}\text { Integral } \\
{[\mathrm{BW} * \% \text { Gait }]}\end{array}$ \\
\hline \multicolumn{7}{|l|}{ OA Assist } \\
\hline Control & $-0.18(0.2)$ & $-0.14(0.2)$ & $-13(8)$ & $0.22(0.2)$ & $0.1(0.2)$ & $15(10)$ \\
\hline $\mathrm{OA}$ & $-0.25(0.2)$ & $-0.13(0.3)$ & $-17(7)$ & $0.16(0.2)$ & $0.2(0.2)$ & $19(9)$ \\
\hline \multicolumn{7}{|l|}{ OA Adjuster 3} \\
\hline Control & $-0.13(0.2)$ & $-0.13(0.2)$ & $-8(8)$ & $0.08(0.2)$ & $0.03(0.2)$ & $8(7)$ \\
\hline $\mathrm{OA}$ & $-0.19(0.2)$ & $-0.02(0.3)$ & $-10(6)$ & $0.04(0.2)$ & $0.09(0.2)$ & $10(7)$ \\
\hline
\end{tabular}

Bold: significant change in joint load due to bracing (Sidak post-hoc test, $\mathrm{P}<0.05$ ) 


\section{Reference List}

[1] Felson DT. Osteoarthritis as a disease of mechanics. Osteoarthr Cartil 2013;21:10-5. doi:10.1016/j.joca.2012.09.012.

[2] Bitton R. The economic burden of osteoarthritis. Am J Manag Care 2009;15:S230-5.

[3] London NJ, Miller LE, Block JE. Clinical and economic consequences of the treatment gap in knee osteoarthritis management. Med Hypotheses 2011;76:887-92. doi:10.1016/j.mehy.2011.02.044.

[4] Waller C, Hayes D, Block JE, London NJ. Unload it: the key to the treatment of knee osteoarthritis. KneeSurgSports TraumatolArthrosc 2011.

[5] Wise BL, Niu J, Yang M, Lane NE, Harvey W, Felson DT, et al. Patterns of compartment involvement in tibiofemoral osteoarthritis in men and women and in whites and African Americans. Arthritis Care Res (Hoboken) 2012;64:847-52. doi:10.1002/acr.21606.

[6] Brouwer RW, van Raaij TM, Verhaar JAN, Coene LNJEM, Bierma-Zeinstra SMA. Brace treatment for osteoarthritis of the knee: a prospective randomized multi-centre trial. Osteoarthritis Cartilage 2006;14:777-83. doi:10.1016/j.joca.2006.02.004.

[7] Dennis DA, Komistek RD, Nadaud MC, Mahfouz M. Evaluation of off-loading braces for treatment of unicompartmental knee arthrosis. J Arthroplasty 2006;21:2-8. doi:10.1016/j.arth.2006.02.099.

[8] Horlick SG, Loomer RL. Valgus Knee Bracing for Medial Gonarthrosis. Clin J Sport Med 1993;3:251-5. doi:10.1097/00042752-199310000-00006.

[9] Haladik JA, Vasileff WK, Peltz CD, Lock TR, Bey MJ. Bracing improves clinical outcomes but does not affect the medial knee joint space in osteoarthritic patients during gait. Knee Surg Sports Traumatol Arthrosc 2014;22:2715-20. doi:10.1007/s00167-0132596-7.

[10] Kutzner I, Küther S, Heinlein B, Dymke J, Bender A, Halder AM, et al. The effect of valgus braces on medial compartment load of the knee joint - in vivo load measurements in three subjects. J Biomech 2011;44:1354-60. doi:10.1016/j.jbiomech.2011.01.014.

[11] Steadman JR, Briggs KK, Pomeroy SM, Wijdicks CA. Current state of unloading braces for knee osteoarthritis. Knee Surg Sports Traumatol Arthrosc 2014;24:42-50. doi:10.1007/s00167-014-3305-x.

[12] Pollo FE, Otis JC, Backus SI, Warren RF, Wickiewicz TL. Reduction of medial compartment loads with valgus bracing of the osteoarthritic knee. Am J Sports Med 2002;30:414-21. 
[13] Shelburne KB, Torry MR, Steadman JR, Pandy MG. Effects of foot orthoses and valgus bracing on the knee adduction moment and medial joint load during gait. Clin Biomech (Bristol, Avon) 2008;23:814-21. doi:10.1016/j.clinbiomech.2008.02.005.

[14] Orishimo KF, Kremenic IJ, Lee SJ, McHugh MP, Nicholas SJ. Is valgus unloader bracing effective in normally aligned individuals: implications for post-surgical protocols following cartilage restoration procedures. KneeSurgSports TraumatolArthrosc 2012.

[15] Gaasbeek RD, Groen BE, Hampsink B, van Heerwaarden RJ, Duysens J. Valgus bracing in patients with medial compartment osteoarthritis of the knee. A gait analysis study of a new brace. Gait Posture 2007;26:3-10.

[16] Jones RK, Nester CJ, Richards JD, Kim WY, Johnson DS, Jari S, et al. A comparison of the biomechanical effects of valgus knee braces and lateral wedged insoles in patients with knee osteoarthritis. Gait Posture 2013;37:368-72. doi:10.1016/j.gaitpost.2012.08.002.

[17] Ramsey DK, Briem K, Axe MJ, Snyder-Mackler L. A mechanical theory for the effectiveness of bracing for medial compartment osteoarthritis of the knee. J Bone Joint Surg Am 2007;89:2398-407. doi:10.2106/JBJS.F.01136.

[18] Toriyama M, Deie M, Shimada N, Otani T, Shidahara H, Maejima H, et al. Effects of unloading bracing on knee and hip joints for patients with medial compartment knee osteoarthritis. ClinBiomech (Bristol, Avon) 2011;26:497-503.

[19] Petersen W, Ellermann A, Zantop T, Rembitzki IV, Semsch H, Liebau C, et al. Biomechanical effect of unloader braces for medial osteoarthritis of the knee: a systematic review (CRD 42015026136). Arch Orthop Trauma Surg 2016;136:649-56. doi:10.1007/s00402-015-2388-2.

[20] Fantini Pagani CH, Willwacher S, Kleis B, Brüggemann G-PP, Pagani CHF, Willwacher $\mathrm{S}$, et al. Influence of a valgus knee brace on muscle activation and co-contraction in patients with medial knee osteoarthritis. J Electromyogr Kinesiol 2013;23:490-500. doi:10.1016/j.jelekin.2012.10.007.

[21] Hubley-Kozey CL, Hill N a., Rutherford DJ, Dunbar MJ, Stanish WD. Co-activation differences in lower limb muscles between asymptomatic controls and those with varying degrees of knee osteoarthritis during walking. Clin Biomech (Bristol, Avon) 2009;24:40714. doi:10.1016/j.clinbiomech.2009.02.005.

[22] Astephen JL, Deluzio KJ, Caldwell GE, Dunbar MJ, Hubley-Kozey CL. Gait and neuromuscular pattern changes are associated with differences in knee osteoarthritis severity levels. J Biomech 2008;41:868-76. doi:10.1016/j.jbiomech.2007.10.016.

[23] Nagai K, Yang S, Fu FH, Anderst W. Unloader knee brace increases medial compartment 
joint space during gait in knee osteoarthritis patients. Knee Surg Sports Traumatol Arthrosc 2018;0:0. doi:10.1007/s00167-018-5274-y.

[24] Brandon SCE, Smith CR, Thelen DG. Simulation of Soft Tissue Loading from Observed Movement Dynamics. In: Müller B, Wolf SI, Brueggemann G-P, Deng Z, McIntosh A, Miller F, et al., editors. Handb. Hum. Motion, Cham: Springer International Publishing; 2017, p. 1-34. doi:10.1007/978-3-319-30808-1_172-1.

[25] Bellamy N. The WOMAC Knee and Hip Osteoarthritis Indices: development, validation, globalization and influence on the development of the AUSCAN Hand Osteoarthritis Indices. Clin Exp Rheumatol 2005;23:S148-53.

[26] Roos EM, Klässbo M, Lohmander LS. WOMAC osteoarthritis index. Reliability, validity, and responsiveness in patients with arthroscopically assessed osteoarthritis. Western Ontario and MacMaster Universities. Scand J Rheumatol 1999;28:210-5.

[27] Chew KTL, Lew HL, Date E, Fredericson M. Current evidence and clinical applications of therapeutic knee braces. Am J Phys Med Rehabil 2007;86:678-86. doi:10.1097/PHM.0b013e318114e416.

[28] Hurley ST, Hatfield Murdock GL, Stanish WD, Hubley-Kozey CL. Is there a dose response for valgus unloader brace usage on knee pain, function, and muscle strength? Arch Phys Med Rehabil 2012;93:496-502. doi:10.1016/j.apmr.2011.09.002.

[29] Miller RH, Edwards WB, Brandon SCE, Morton AM, Deluzio KJ. Why don't most runners get knee osteoarthritis? A case for per-unit-distance loads. Med Sci Sports Exerc 2014;46:572-9. doi:10.1249/MSS.0000000000000135.

[30] Neville SR, Brandon SCE, Brown MJ, Deluzio KJ. Validation of method for analysing mechanics of unloader brace for medial knee osteoarthritis. J Biomech 2018:5-10. doi:10.1016/j.jbiomech.2018.05.035.

[31] Barbero M, Merletti R, Rainoldi A. Atlas of Muscle Innervation Zones. Milano: Springer Milan; 2012. doi:10.1007/978-88-470-2463-2.

[32] Luca CJ De, Gilmore LD, Kuznetsov M, Roy SH, De Luca CJ, Gilmore LD, et al. Filtering the surface EMG signal: Movement artifact and baseline noise contamination. J Biomech 2010;43:1573-9. doi:10.1016/j.jbiomech.2010.01.027.

[33] Hubley-Kozey CLL, Deluzio KJJ, Landry SC, McNutt JS, Stanish WDD, Landry CSN, et al. Neuromuscular alterations during walking in persons with moderate knee osteoarthritis. J Electromyogr Kinesiol 2006;16:365-78. doi:10.1016/j.jelekin.2005.07.014.

[34] Cooke TD V, Sled E a, Scudamore RA. Frontal plane knee alignment: a call for standardized measurement. J Rheumatol 2007;34:1796-801. 
[35] Buckland-Wright JC, Macfarlane DG, Williams S a, Ward RJ. Accuracy and precision of joint space width measurements in standard and macroradiographs of osteoarthritic knees. Ann Rheum Dis 1995;54:872-80. doi:10.1136/ard.54.11.872.

[36] Lerner ZF, Haight DJ, DeMers MS, Board WJ, Browning RC. The effects of walking speed on tibiofemoral loading estimated via musculoskeletal modeling. J Appl Biomech 2014;30:197-205. doi:10.1123/jab.2012-0206.

[37] Winby CR, Gerus P, Kirk TB, Lloyd DG. Correlation between EMG-based co-activation measures and medial and lateral compartment loads of the knee during gait. Clin Biomech (Bristol, Avon) 2013;28:1014-9. doi:10.1016/j.clinbiomech.2013.09.006.

[38] Halaki M, Ginn K. Normalization of EMG Signals: To Normalize or Not to Normalize and What to Normalize to? In: Naik GR, editor. Comput. Intell. Electromyogr. Anal. - A Perspect. Curr. Appl. Futur. Challenges, InTech; 2012, p. 175-94. doi:10.5772/49957.

[39] Arnold EM, Ward SR, Lieber RL, Delp SL. A model of the lower limb for analysis of human movement. Ann Biomed Eng 2010;38:269-79. doi:10.1007/s10439-009-9852-5.

[40] Brandon SCE, Miller RH, Thelen DG, Deluzio KJ. Selective lateral muscle activation in moderate medial knee osteoarthritis subjects does not unload medial knee condyle. J Biomech 2014;47:1409-15. doi:10.1016/j.jbiomech.2014.01.038.

[41] Clouthier AL, Hassan EA, Brandon SCE, Campbell A, Rainbow MJ, Deluzio KJ. Identification of good candidates for valgus bracing as a treatment for medial knee osteoarthritis. J Orthop Res 2017. doi:10.1002/jor.23663.

[42] Delp SL, Anderson FC, Arnold AS, Loan P, Habib A, John CT, et al. OpenSim: opensource software to create and analyze dynamic simulations of movement. IEEE Trans Biomed Eng 2007;54:1940-50. doi:10.1109/TBME.2007.901024.

[43] Hicks JL, Uchida TK, Seth A, Rajagopal A, Delp S. Is my model good enough? Best practices for verification and validation of musculoskeletal models and simulations of human movement. J Biomech Eng 2015;137:020905. doi:10.1115/1.4029304.

[44] Thelen DG, Anderson FC, Delp SL. Generating dynamic simulations of movement using computed muscle control. J Biomech 2003;36:321-8.

[45] Brandon SCEE, Deluzio KJ. Robust features of knee osteoarthritis in joint moments are independent of reference frame selection. Clin Biomech 2011;26:65-70. doi:10.1016/j.clinbiomech.2010.08.010.

[46] Happee R, Van der Helm FCT. The control of shoulder muscles during goal directed movements, an inverse dynamic analysis. J Biomech 1995;28:1179-91. doi:10.1016/00219290(94)00181-3. 
[47] Lerner ZF, DeMers MS, Delp SL, Browning RC. How tibiofemoral alignment and contact locations affect predictions of medial and lateral tibiofemoral contact forces. J Biomech 2015;48:644-50. doi:10.1016/j.jbiomech.2014.12.049.

[48] Winby CR, Lloyd DG, Besier TF, Kirk TB. Muscle and external load contribution to knee joint contact loads during normal gait. J Biomech 2009;42:2294-300.

[49] Richards C, Higginson JS. Knee contact force in subjects with symmetrical OA grades: differences between OA severities. J Biomech 2010;43:2595-600.

[50] Kutzner I, Heinlein B, Graichen F, Bender A, Rohlmann A, Halder A, et al. Loading of the knee joint during activities of daily living measured in vivo in five subjects. J Biomech 2010;43:2164-73.

[51] Kutzner I, Trepczynski A, Heller MO, Bergmann G. Knee adduction moment and medial contact force--facts about their correlation during gait. PLoS One 2013;8:e81036. doi:10.1371/journal.pone.0081036.

[52] Zhao D, Banks SA, Mitchell KH, D'Lima DD, Colwell Jr. CW, Fregly BJ. Correlation between the knee adduction torque and medial contact force for a variety of gait patterns. JOrthopRes 2007;25:789-97.

[53] Fregly BJ, Besier TF, Lloyd DG, Delp SL, Banks SA, Pandy MG, et al. Grand challenge competition to predict in vivo knee loads. JOrthopRes 2012;30:503-13.

[54] Demers MS, Pal S, Delp SL. Changes in tibiofemoral forces due to variations in muscle activity during walking. J Orthop Res 2014;32:769-76. doi:10.1002/jor.22601.

[55] Miller RH, Brandon SCE, Deluzio KJ. Predicting Sagittal Plane Kinematics that Minimize the Knee Joint Contact Force. 17th Bienn. Meet. Can. Soc. Biomech., Burnaby, British Columbia, Canada: 2012. doi:10.1098/rspb.2011.2015.

[56] Sritharan P, Lin YC, Pandy MG. Muscles that do not cross the knee contribute to the knee adduction moment and tibiofemoral compartment loading during gait. JOrthopRes 2012;30:1586-95.

[57] Arazpour M, Bani MA, Maleki M, Ghomshe FT, Kashani R V, Hutchins SW. Comparison of the efficacy of laterally wedged insoles and bespoke unloader knee orthoses in treating medial compartment knee osteoarthritis. ProsthetOrthotInt 2012.

[58] Schmalz T, Knopf E, Drewitz H, Blumentritt S. Analysis of biomechanical effectiveness of valgus-inducing knee brace for osteoarthritis of knee. J Rehabil Res Dev 2010;47:41929.

[59] Hewett TE, Noyes FR, Barber-Westin SD, Heckmann TP. Decrease in knee joint pain and increase in function in patients with medial compartment arthrosis: a prospective analysis 
of valgus bracing. Orthopedics 1998;21:131-8.

[60] Clouthier AL, Hassan EA, Brandon SCE, Campbell A, Rainbow MJ. Identification of good candidates for valgus bracing as a treatment for medial knee osteoarthritis 2016; In Review.

[61] Lloyd DG, Besier TF. An EMG-driven musculoskeletal model to estimate muscle forces and knee joint moments in vivo. J Biomech 2003;36:765-76.

[62] Gerus P, Sartori M, Besier TF, Fregly BJ, Delp SL, Banks SA, et al. Subject-specific knee joint geometry improves predictions of medial tibiofemoral contact forces. J Biomech 2013;46:2778-86. doi:10.1016/j.jbiomech.2013.09.005.

[63] Saliba CM, Brandon SCE, Deluzio KJ. Sensitivity of medial and lateral knee contact force predictions to frontal plane alignment and contact locations. J Biomech 2017;57:125-30. doi:10.1016/j.jbiomech.2017.03.005.

[64] Adouni M, Shirazi-Adl A. Partitioning of knee joint internal forces in gait is dictated by the knee adduction angle and not by the knee adduction moment. J Biomech 2014;47:1696-703. doi:10.1016/j.jbiomech.2014.02.028.

[65] Lloyd DG, Buchanan TS. Strategies of muscular support of varus and valgus isometric loads at the human knee. J Biomech 2001;34:1257-67.

[66] Dhaher YY, Tsoumanis AD, Houle TT, Rymer WZ, Dhaher YY, Tsoumanis AD, et al. Neuromuscular Reflexes Contribute to Knee Stiffness During Valgus Loading Neuromuscular Reflexes Contribute to Knee Stiffness During Valgus Loading 2014:2698-709.

[67] Lenhart RL, Kaiser J, Smith CR, Thelen DG. Prediction and Validation of LoadDependent Behavior of the Tibiofemoral and Patellofemoral Joints During Movement. Ann Biomed Eng 2015;43:2675-85. doi:10.1007/s10439-015-1326-3.

[68] Marra MA, Vanheule V, Rasmussen J, Verdonschot NJJ, Andersen MS, Fluit R, et al. A Subject-Specific Musculoskeletal Modeling Framework to Predict in Vivo Mechanics of Total Knee Arthroplasty. J Biomech Eng 2014;137:020904. doi:10.1115/1.4029258.

[69] Walter JP, D’Lima DD, Colwell Jr. CW, Fregly BJ. Decreased knee adduction moment does not guarantee decreased medial contact force during gait. JOrthopRes 2010;28:134854 .

[70] Fregly BJ, D'Lima DD, Colwell Jr. CW. Effective gait patterns for offloading the medial compartment of the knee. JOrthopRes 2009;27:1016-21. 